\title{
AUGMENTED REALITY AND HUMAN FACTORS APPLICATIONS FOR THE NEUROSURGICAL OPERATING ROOM
}

\author{
By
}

\begin{abstract}
Nhu Quynh Nguyen
Bachelor of Engineering, Ryerson University, 2016
\end{abstract}

A thesis,

presented to Ryerson University

in partial fulfilment of the

requirements for the degree of

Master of Applied Science

in the program of

Electrical and Computer Engineering

Toronto, Ontario, Canada, 2019

(C) Nhu Nguyen, 2019 


\section{AUTHOR'S DECLARATION FOR ELECTRONIC SUBMISSION OF A THESIS}

I hereby declare that I am the sole author of this thesis. This is a true copy of the thesis, including and required final revisions, as accepted by my examiners.

I authorize Ryerson University to lend this thesis to other institutions or individuals for the purpose of scholarly research.

I further authorize Ryerson University to reproduce this thesis by photocopying or by other means, in total or in part, at the request of other institutions or individuals for the purpose of scholarly research.

I further authorize Ryerson University to reproduce this thesis by photocopying or by other means, in total or in part, at the request of other institutions or individuals for the purpose of scholarly research. I understand that my thesis may be made electronically available to the public. 


\begin{abstract}
Augmented Reality and Human Factors Applications for the Neurosurgical Operating Room
\end{abstract}

\author{
Nhu Quynh Nguyen \\ Master of Applied Science \\ Electrical and Computer Engineering \\ Ryerson University, 2019
}

The virtual overlay of patient-specific anatomies onto a surgical site through Augmented Reality (AR) technologies has been thought to be a potentially ideal neuronavigational system for use in neurosurgery. Although impressive and futuristic, there are many design considerations that must be taken into account, including surgeon reception, perceived utility, intuitive control and manipulation design, and overall system accuracy during surgery. To implement AR into the neurosurgical Operating Room (OR), a gradual approach of evolutionary design to ensure widespread adoption may be considered. This thesis presents a potential pathway for the introduction of AR technologies into the neurosurgical OR.

The thesis is divided into three parts: incorporation of AR features into existing platforms for improved functionality and introduction of AR concepts to surgical environments, observation and evaluation of surgeon perception of AR overlays and AR headsets to inform display methods and designs, and quantification of virtual object placement accuracy in a clinical environment. The findings presented show that AR integrated systems improve OR workflow when conventional tracked tools are unavailable, user preference of AR overlays onto the surgical site change depending on operator experience level, and the placement accuracy of state-of-the-art AR head mounted displays are suitable for presurgical planning and very close to accuracy needed for surgical guidance. These three elements are key to developing a 
pathway for adoption of AR technologies in the OR, and help to inform designs for future headsets to assist surgeons and improve patient care. 


\section{ACKNOWLEDGEMENTS}

My master's research has been nothing short of interesting. I've met so many great researchers and people who are so passionate to change the world with their work and improve the lives of so many people. I would like to take a moment to remember and thank all the people who have been so helpful and supportive through this journey as I grew as a person and as a researcher.

First, I would like to thank my supervisors Dr. Dimitrios Androutsos and Dr. Victor X.D. Yang for their mentorship and guidance throughout my degree. I first met Dr. Androutsos in my undergraduate degree as my professor for Discrete Signals and Systems. The tools that I learned from this course have heavily assisted me in my graduate degree as these fundamentals extended incredibly far to further fields. I will never forget how difficult that exam was. I failed it- but this failure also taught me that grades aren't everything, it doesn't null all the things that I have learned and that students are much more than just grades and numbers. I met Dr. Yang as a summer student in the Biophotonics and Bioengineering Laboratory (BBL)- also during my undergraduate degree. With many hours in the operating room and the research labs, it was such a beautiful environment to be immersed in. I could see the intersection of engineering and a real-world medical setting. He has given me many learning opportunities and introduced me to the wonderful BBL team - my second family.

When I started, I was assisting Dr. Jakubovic with his $\mathrm{PhD}$ research at the time by evaluating the accuracy of pedicle screw insertion (a.k.a. "screwing around") a famously tedious job in the BBL at the time. I followed in his footsteps when I received my own summer students to mentor later in my master's degree. Thank you for encouraging me to pursue research and seeing potential in me years ago.

I would like to thank Philips Lai, Ryan Deorajh, and Jillian Cardinell- my Vision Team. I'll never forget the late evenings we shared at the lab and the incredible amount of eyestrain we all suffered together from 
our head mounted displays and inordinate amount of computer screen time. We always joked about how we'd lose our vision later in life and slowly transition into the "Hearing or Olfactory Team". I've learned so much from you all- about myself, life, resiliency, and the multimedia research we executed together. You will all go on to do so many great things and I am so thankful for having you as part of my team.

I would also like to thank the other members of the BBL Joel Ramjist, Yuta Dobashi, Jamil Jivraj, Dexter Barrows, Sophia Barrows, Christopher Pasarakovski, Daipayan Guha, Stefano Priola, Andrew Marques, Robnier Reyes, Shaurya Gupta, Chaoliang Chen, Weisong Shi, and Dorian Chen for teaching me about experimental design, proofreading my thesis, scientific communication, and how to push the boundaries of my comfort zone. You all inspired and motivated me to continue my research on my most difficult days and helped me with my mental health when I needed you most. You taught me that research is hard but so rewarding. Most importantly, you all have taught me how much fun research can be! I'll never forget our much-needed outings to destress from our chaotic days.

Not only did Ryerson University become my second home, but also Sunnybrook Health Sciences Centre. Thank you to my desk mates Elahe Marandi, Morgan Koo, Patrick Ji, Sairah Mirza, and Amin Banihashemi for your comedic humor. Although we did not work together directly, it was so much fun having you all to talk to. Sunnybrook really did become a warm research environment. You are all such kind and generous people. 
Finally, I would like to thank my family for their unconditional love and support. I would like to especially thank Phuong Nguyen for putting up with my complaining and inane babbling. Special thanks also to my parents for making sure that I had a good lunch and ride from the bus station every single day. 


\section{DEDICATION}

Dedicated to my parents and my sister who have always told me to work hard, to love what I do, and never give up. 


\section{TABLE OF CONTENTS}

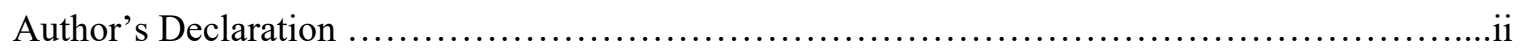

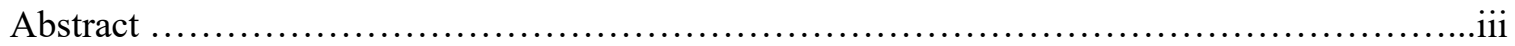

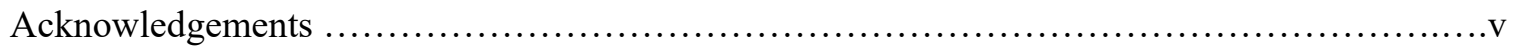

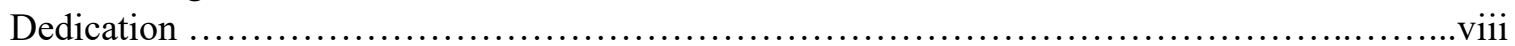

Table of Contents ..................

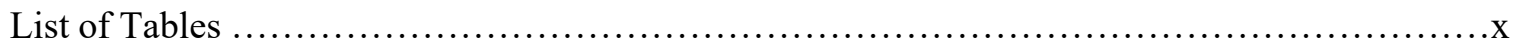

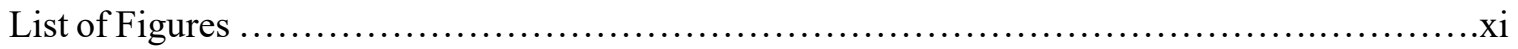

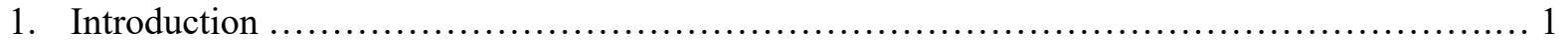

1.1 Human Computer Interaction ..................................................... 1

1.1.1 The Operating Room Workflow .............................................. 1

1.1.2 Human Factors and Navigation Systems ................................... 5

1.1.3 Human Factors and Augmented Reality Navigation Systems .................... 10

1.1.4 State of the Art Augmented Reality Systems in Neurosurgery ..................... 13

1.2 Thesis Motivation and Contributions ................................................

1.2.1 Guidance of Pedicle Screw Insertion Using AR Trajectories ........................ 17

1.2.2 Surgeons and the Perception of Augmented Reality ................................. 18

1.2.3 Augmented Reality Placement Accuracy ....................................... 18

2. Guidance of Pedicle Screw Insertion Using Augmented Reality Trajectories ......................20

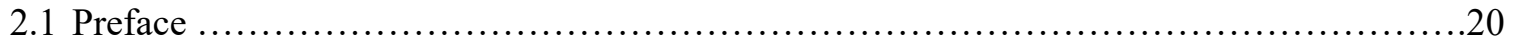

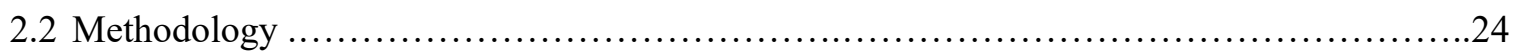

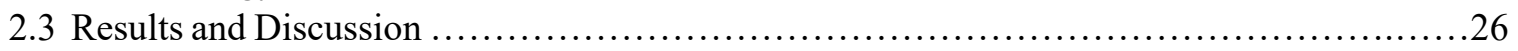

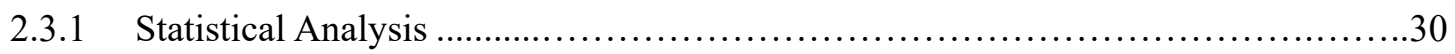

2.3.2 Practical Interpretation and Limitations ....................................... 31

3. Surgeons and the Perception of Augmented Reality in the Operating Room ......................35

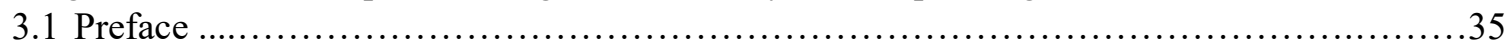

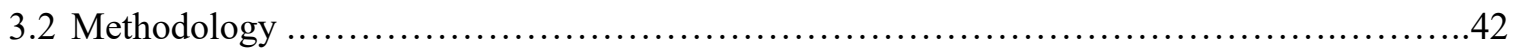

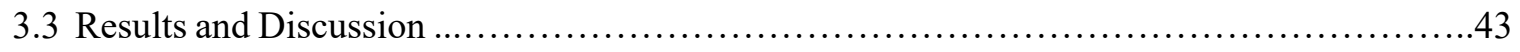

3.3.1 Practical Interpretation and Limitations ......................................... 44

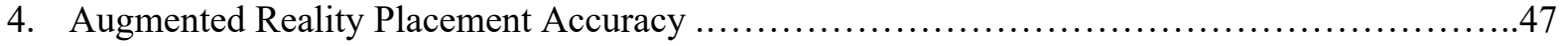

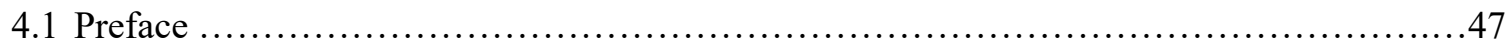

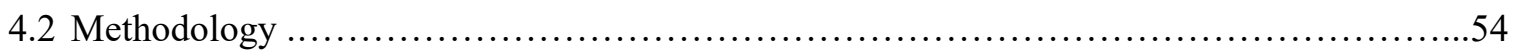

4.2.1 The Measurement Process ...................................................... 58

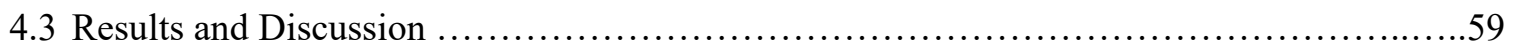

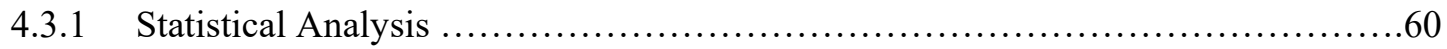

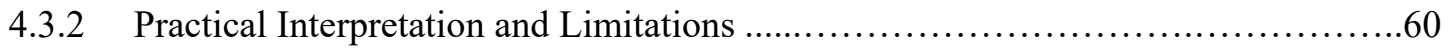

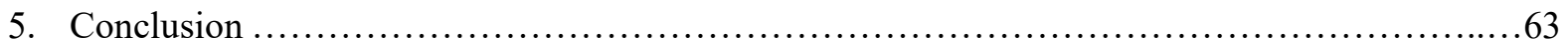

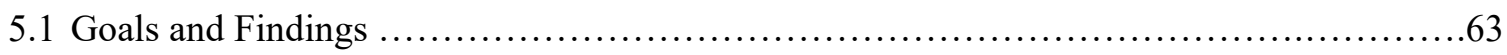

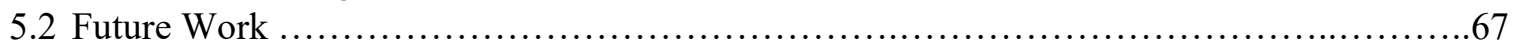

Appendix ......................................................................6

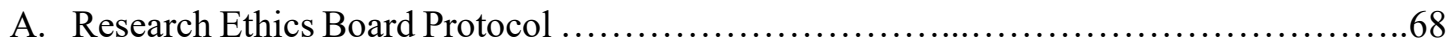

B. Data Collection Form ........................................................... 76

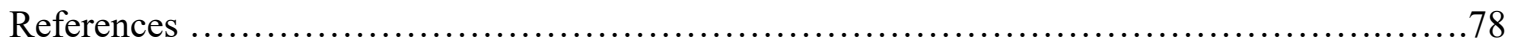




\section{LIST OF TABLES}

Table 1: Average magnitude of safe zones seen in the phantom tests. Average values were found for different regions of the spine (cervical, thoracic, lumbar, and sacral)

Table 2: Average magnitude of safe zones seen in the clinical tests. Average values were found for different regions

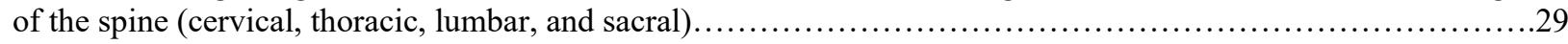

Table 3: Preferred transparency values and colours for each participant...................................43

Table 4: Phantom Experiment Displacement Error of Virtual Object Placement with Respect to Real Object........59

Table 5: Phantom Experiment Rotational Error of Virtual Object Placement with Respect to Real Object............59

Table 6: Clinical Experiment Displacement Error of Virtual Object Placement with Respect to Real Object........59

Table 7: Clinical Experiment Rotational Error of Virtual Object Placement with Respect to Real Object...........60 


\section{LIST OF FIGURES}

Figure 1: The image on the right displays the navigation screen of the 7D Machine vision Image Guided Surgical (MvIGS) system where the blue line is the tracked tool and the green line is the intended trajectory.....

Figure 2: Registration process using an IR tracking system for surgical navigation. A) A 3D model is constructed based on CT data B) The known configuration of IR balls (red dots) on tracked tools is known and highly visible to the IR tracking system when in the working volume of the tracking system C) Using the tracked tool, points are identified on the surgical site D) Corresponding points on the surgical site are selected on the 3D model from A). E) These points are matched as best as possible. User is now ready to navigate.

Figure 3: There is a visual disjunct between the surgical navigation screen (left) and the surgical site (right). The blue trajectory is the position of the tracked tool. The tool is the only piece of information linking these two views..8 Figure 4: The workflow prevents surgeons from focusing on the surgical site. Surgeons must also keep their hands as still as possible to not lose the intended tool position. This constant changing view from the screen to the surgical

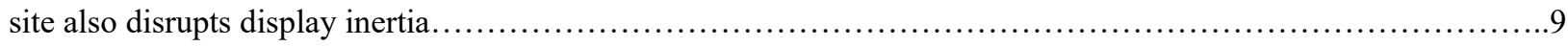

Figure 5: A) CT images are taken of the patient to diagnose and form a treatment plan. B) Based on the CT images, a $3 \mathrm{D}$ reconstruction can be made based on the grey levels for image segmentation. C) Using an AR HMD, the 3D

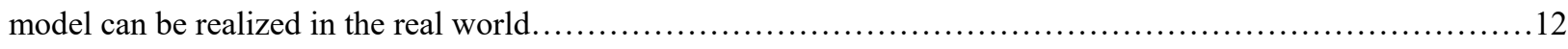

Figure 6: Scopus AR Navigation system uses a planned trajectory to create a path for guidance in conjunction with the conventional IR tracking system. The AR view on the left is also an example of the kind of view the surgeon has from using the laparoscope.

Figure 7: ARSN system from Philips. This system uses x-ray images and displays them on top of the surgical site

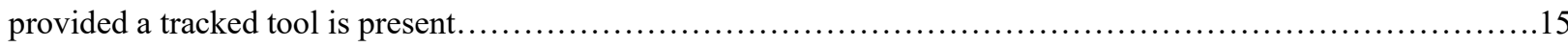

Figure 8: The image on the right displays the navigation screen of the 7D MvIGS system where the blue line is the tracked tool and the green line is the intended trajectory. On the right is the overhead view of a standard surgical site for spine. There is a disconnect in transferring the outlined trajectory to the surgical site when using an unnavigated tool where the view on the left pictured here is no longer available.

Figure 9: There are many possible causes for losing navigation regarding placement of the IR tracking system (A) The optimal spectrum is not visible to users because it is IR light. (B) Ideal navigation placement with respect to the surgical site (C) Non-optimal placement, the IR reflectors on the tool are outside the invisible range (D) Nonoptimal placement, the surgical site is out of range. 
Figure 10: (Left) Angle Measurement system notation. The blue trajectories shown by the tracked tool are considered the reference trajectories. The dotted lines are used to describe different types of breaches. The red, pink, yellow and green lines represent medial, lateral, superior and inferior breaches respectively. (Right) $\alpha_{1}$ shows the angle made from a medial breach and $\alpha_{2}$ shows a lateral breach; these zones are noted by red and pink zones respectively. $\beta_{1}$ shows a superior breach and $\beta_{2}$ shows an inferior breach; these zones are green and yellow respectively. The lines differentiating in between these safe zones is considered the correct trajectory.....

Figure 11: The light head of the 7D MvIGS system. Inside consists of the infrared tracker and the stereoscopic cameras. The location of the cameras is indicated by the red circles. This system is designed to overlook the surgical site to maintain the operating room workflow....

Figure 12: Phantom Data collected from C2 to S1. A) Left side of the spine, $\alpha_{1}+\alpha_{2}$ angles. B) Left side of the spine, $\beta_{1}+\beta_{2}$ angles. C) Right side of the spine, $\alpha_{1}+\alpha_{2}$ angles C) Right side of the spine, $\beta_{1}+\beta_{2}$ angles.................26 Figure 13: Clinical Data collected from C2 to S1. A) Left side of the spine, $\alpha_{1}+\alpha_{2}$ angles. B) Left side of the spine, $\beta_{1}+\beta_{2}$ angles. C) Right side of the spine, $\alpha_{1}+\alpha_{2}$ angles C) Right side of the spine, $\beta_{1}+\beta_{2}$ angles.

Figure 14: Mean Phantom (left) vs Mean Clinical (right) Radiological Angles and Camera Angles. We see that in almost all cases, the mean camera angles are larger than the mean radiological angles.....

Figure 15: A, B, and C describe different views of a correct trajectory. D, E, and F describe different views of a superior breach. In both cases, the light head cameras are oriented parallel to the spine and the tool moves along this plane between trajectories. When comparing B and E, we see that the overhead cameras appear similar. However, the radiological angles seen in A and D show a difference of $13^{\circ}$ in the sagittal plane. $13^{\circ}$ is more than enough of a deviation to potentially cause a breach.

Figure 16: Comparison between minimally invasive spine surgery (left) and open spine surgery (right). The opening for MIS is only about $2.5 \mathrm{~cm}$ in comparison to the large surgical site created from open spine surgery [39].....

Figure 17: A) A standard surgical site for MIS. We can see that the site is very small and there are very few visual cues informing the surgeon where they are in relation to patient anatomy. B) Overlaying the virtual information from a CT scan allows surgeons to see where in the spine they are operating on. The mixed reality view allows surgeons to see within the patient body without a large surgical site C) There are numerous rendering methods available today to determine how best to show the virtual information without risking inattentional blindness, including wireframe methods visualized here.

Figure 18: The sphere on the left is an example of a wireframe model. Notice that the triangles composing the mesh are outlined in green, but the internal area of the triangles are transparent. The sphere on the right is opaque, no

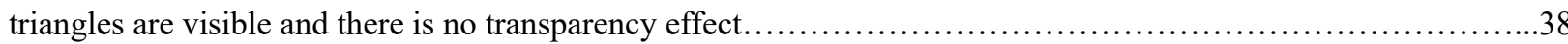

Figure 19: An example of a HUD for a pilot where minimal information is displayed while maintaining a clear view of the skies. 
Figure 20: A) Viewing a real-world object results in matching accommodation and vergence distances. B) A virtual display shows an object close to the observer which results in mismatch as the eyes converge on the display. C) A virtual display showing a far object also results in mismatch. Both forms of mismatch shown in panels $\mathrm{B}$ and $\mathrm{C}$ result in poor stereoacuity. Figures are adapted from [51]

Figure 21: An example of the spatial mapping capabilities in the angiography suite during an interventional neuroradiology case. The patient anatomy shown in purple is in the process of being placed onto the physical patient. The left panel is the normal mixed reality view while the right panel is the spatial mapping capability of the HoloLens used to better understand the surfaces in the room.

Figure 22: Registration process for 3-point correspondence. A) upon start up, the application activates spatial mapping at the highest possible resolution. The yellow box displays the virtual patient anatomy with virtual points pre-decided. The orange box shows the three real world points. B) The user places the real points onto the physical patient corresponding to the pre-decided points shown in the pink box C) Best fit is determined based on the centroids and normals of the virtual and real coordinates.

Figure 23: Various phantoms used for placement testing of all methods. First row from left to right are the head phantom and a percutaneous phantom. These two phantoms are the relatively smaller level of detail. The bottom row, a phantom spine and a calibration block have a greater level of detail.

Fig. 24. Virtual patient anatomy is overlaid in white and the real patient anatomy is shown in dark grey. The vectors outlined in red show the rotational error between the real and virtual models (determined by the dot product). The blue line shows the displacement error between salient features (in this case, the nose tips).

Figure 25: Left and Right camera overhead view of the surgical site during T6 and T7 fusion. The green line imposed onto the surgical site is the ideal pedicle screw trajectory. The screwdriver being used here is untracked and following the pedicle screw . .64

Figures 26: Left image - Ideal representation for surgeons with experience greater than PGY3. Right image Example of contrasting colours for individuals with experience less than PGY3. 


\section{INTRODUCTION}

\subsection{Human Computer Interaction}

To determine if something is inherently useful or not, human computer interaction is of utmost importance. Engineers have shown the ability to design incredible technologies which push the boundaries of existing systems; however, if these systems are built in such a way that the main user group cannot use them properly or understand the purpose, they are often forgotten. Human Computer Interaction (HCI) is often a powerful concept which is highly underestimated. Well implemented HCI is often seamless but can produce a huge impact. To study the way that humans operate and interact with technology is a crucial step to developing widely adopted systems. The Operating Room (OR) is an interesting environment to examine and has seen impressive technologies come and go.

\subsubsection{The Operating Room Workflow}

Spine surgery is a prime example of a surgery that requires multiple devices employed at various stages throughout a procedure. Some of these include ultrasound, CT scanners, x-ray systems, neuromonitoring systems, etc. One of the most interesting technologies are surgical navigation systems. These systems are capable of submillimeter accuracy and are designed to track specific tools such that users can orient themselves with the patient anatomy. These systems are often used for cranial procedures but can also be used for spine [1]. With this system, users can determine if there are any vital organs or structures which should be handled with care or avoided. Figure 1 shows a standard view from a surgical navigation screen. Here, we see the patient anatomy shown in relation to the tool being used by the surgeon. The view of the patient anatomy changes dynamically in real time as the tool tip moves within the visible volume of the surgical navigation device. 


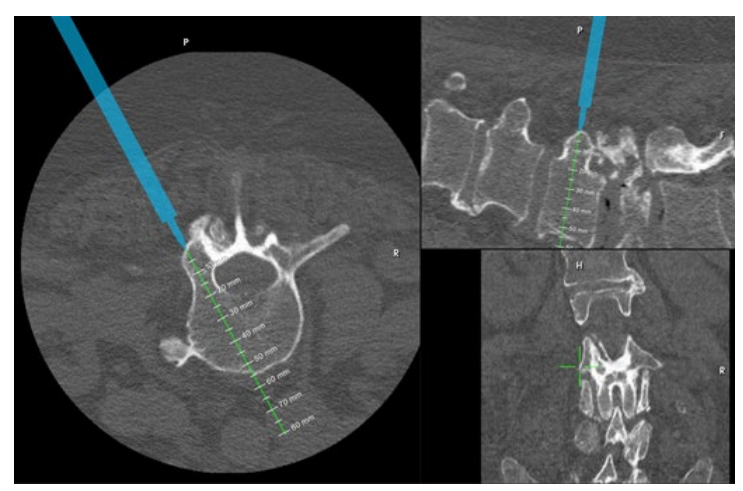

Figure 1: The image on the right displays the navigation screen of the 7D Machine vision Image Guided Surgical (MvIGS) system where the blue line is the tracked tool and the green line is the intended trajectory.

The ability to see the underlying patient anatomy is of great value during surgery. Without navigation systems, users would only have the view of the surgical site and a series of medical images. It is quite difficult to spatially relate the medical images to the surface of the surgical site without a visual connection between the two sets of information together. The surgeon must entirely depend on their memory and their understanding of human anatomy to prevent puncturing vital organs and structures lying underneath the surface. After a mental orientation is established between the two sets of information, the user is then required to carry out the procedure. In the event of pedicle screw insertion, the screw is inserted into the pedicle. This method of implant placement is also known as the anatomic free-hand technique [2]. By using surgical navigation devices and tracked tools, users can relate the surgical site to the medical images. When the user holds the tracked tool in different orientations, different views of the medical scans are displayed relative to the tool by using infrared (IR) technology [3]. By using stereoscopic IR cameras and IR markers placed on the surgical tool, the CAN system can recognize the tool and understand its rotation and displacement if the tool is within the view of the cameras. The IR cameras are also configured with an IR light source so that the reflectors are highly visible to the cameras [4]. Knowing the unique IR marker configuration on the tool, the system is also aware of the location of the tool tip with respect to these markers which is important for registration. Registration refers to the relationship between the virtual patient images and the physical spatial world. In many navigation systems, registration is done through selecting points on the physical patient using a tracked tool and aligning these points to equivalent points selected from pre-operative images (such as CT or MR) on the navigation system [4]. From there, the navigation system performs a best fit between the virtual points outlined on the patient images and the set of points outlined on the physical patient. 


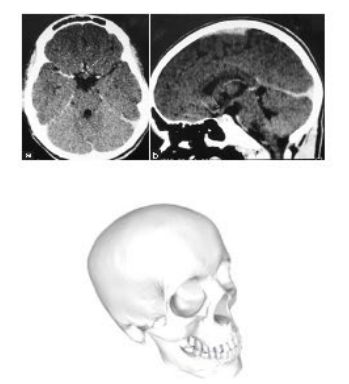

(A)

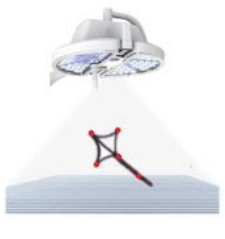

(B)

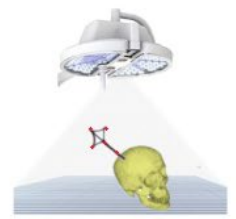

(C)
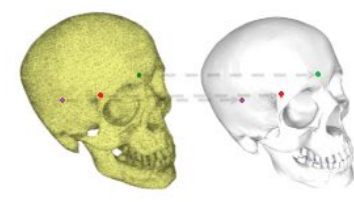

(D)

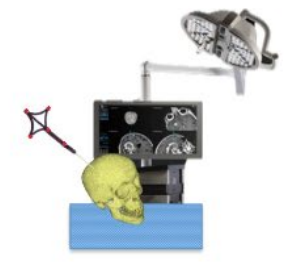

(E)

Figure 2: Registration process using an IR tracking system for surgical navigation. A) A 3D model is constructed based on CT data B) The known configuration of IR balls (red dots) on tracked tools is known and highly visible to the IR tracking system when in the working volume of the tracking system C) Using the tracked tool, points are identified on the surgical site D) Corresponding points on the surgical site are selected on the 3D model from A). E) These points are matched as best as possible. User is now ready to navigate.

Since these medical images used during registration are not acquired in real time during the surgery, there is a point where surgical navigation can no longer be used during the case because the patient anatomy has been altered too far from the time when the medical images were acquired. For example, during tumour resection in cranial surgery, brain shift often occurs, changing the position of underlying anatomy and rending navigation inaccurate [5]. The changes in the patient anatomy between the time of imaging and the time when the surgery starts is considered negligible because neuro anatomy does not tend to shift without the introduction of trauma or surgical intervention.

To compensate for the changes during surgery, some navigation systems will have additional components to provide real time images. One example is the Medtronic Stealth Station which has a mobile CT scanner called the $\mathrm{O}-\mathrm{arm}^{\mathrm{TM}}$. If the surgeons feel as though the patient anatomy has shifted too much from the original patient scans, a more updated series of images can be taken to reassess the current situation. Alternatively, for systems without a CT scanner, fluoroscopic x-ray images are also used to check the patient anatomy during surgery, but only provide $2 \mathrm{D}$ images and use less radiation compared to a CT scan. Surgical navigation systems are also unable to register to the 2D image as it is not a series of images like CT scans. Both the CT and the x-ray machines expose the hospital staff and patient to radiation to acquire up to date images. Hospital staff are either required to leave the room while the $\mathrm{x}$-ray machines are in use or wear heavy and cumbersome lead vests to stay in the OR.

Without surgical navigation, it would be incredibly difficult to operate with accuracy on the submillimeter level. Specifically, reported incident rates of breaching the spinal column ranges from $3-55 \%$ for the 
thoracic spine, and 5-41\% for lumbar spine using standard acceptable free-hand techniques [6][7][8][9]. Surgical navigation has been shown to reduce breach rates to less than $10 \%$ [10].

Navigation through the pedicle requires great precision, with medial and lateral breaches potentially damaging the spinal column or major vasculature, respectively, and with screws that are inserted too deep breaching the vertebral body and potentially perforating key organs including the lungs and aorta. Only $11 \%$ of spine surgeons in Europe and North America use navigation daily. Routine users are surgeons who have a high volume of patients, neurological surgeons. and surgeons who have a busy minimally invasive surgery (MIS) practice [1]. The main reasons for not using navigation is because of the steep learning curve, lack of equipment, high costs, and potentially increased time due to registration. Although the primary user group is aware that surgical navigation is highly valuable, existing systems fail to meet their expectations in ease of use and integration into the existing OR workflow [1].

One of the slowest portions in the workflow once navigation is introduced in the registration process. Surgeons are required to send dozens of coordinates to the system to notify the system of the location of certain landmarks on the physical patient. This process can take up to 15 minutes to complete. In the event where a patient in the operating room is suffering from severe blood loss, 15 minutes to simply set up a device is far too long. Even for less severe cases, time spent in an operating room can cost a lot considering the number of personnel, disposables being used, etc. Each minute in an operating room costs the hospital approximately \$62 USD on average, which means \$930 USD would be the cost to simply set up the device not including other associated costs such as anesthesiology and the neurosurgeon $[11][12][13]$.

Overall, existing surgical navigation devices are incredibly accurate and can increase patient safety in spine surgery. However, from the perspective of the user, these devices are potentially valuable but generally difficult to use and do not work with the existing constraints in the operating room where high stress scenarios are common. This is where one can see that HCI plays a large role in the adoption and efficacy of new medical devices in the OR. The end users and the potential use cases are of utmost importance when designing systems to ensure they are seamlessly integrated and can be easily adopted [14]. 


\subsubsection{Human Factors and Navigation Systems}

Human Computer Interaction (HCI) or human factors is a very complex topic. The discipline was formalized during world war II. A commonly mentioned example is the redesign of airplane cockpits. Initial designs caused pilots to unintentionally bail out of their planes. By redesigning the seat ejection mechanism from a handle to a throttle, there was a significant decrease in accidental ejections [14][15]. The mental state of an individual who is calm under safe conditions is very different when they are stressed and can potentially lose their lives. Under training and supervision, it was easy to avoid the handle. During stressful situations, this may not have been as straight forward. One can argue that the pilots could have learned to adapt better to the design, or that maybe the pilots are simply unintelligent and unable to learn the various controls. If they could learn where the essential controls are and what certain handles do, why can't they do it again?

The study of HCI arises from the realization that the user should not have to adapt to the interface, but the interface should serve the user. In the case of the airplane pilots; build controls that are natural to them and their normal experiences as a pilot. By studying human behavior and performance, general guidelines were developed. Usually, people consider these guidelines to be common sense in design, but they are not necessarily simple to apply. If this sense is so common, why are there so many unintuitive user interfaces? Applying vague guidelines to specific use cases such as surgical navigation or air pilot cockpit design is not as transparent as one may think [14]. An analysis of the user interface found in surgical navigation systems from an HCI perspective can further explain in depth where some of the specific underlying problems are. This analysis based on guidelines can also help to explain the poor adoption rate in the operating room despite having an impressively accurate navigation system.

User-centred design is one of the most fundamental concepts when considering HCI in design [14]. Understanding the thoughts of the user and how they may naturally view or interact with a system is important in creating a simple and intuitive interface for a specific user group. In the case of surgical navigation systems, it is seen that there are some specific guidelines that are followed very closely, and some that are not, which are potential causes for low adoption rate by surgeons who operate in spinerelated surgeries. The guidelines for user design are by no means concrete and can even potentially conflict or overlap with one another in some cases. They were made to be suggestions which the designer may choose to follow when appropriate given the scenario at their discretion.

One example of how the guidelines are used is in the navigation screen itself, which displays the patient anatomy in an interpreted 3D space. While clinicians are completing their residency, they are taught how to read these images carefully and interpret certain structures, landmarks, anatomy etc. During treatment 
planning, surgeons will use CT or MRI images to determine what the next steps are to ensure the highest standard of patient care. When operating, the same images are displayed in the surgical navigation system when using navigated tools in the same format. The same thought process and spatial thinking from the treatment planning stage is resumed when the surgery starts because the same images are used in both stages of patient care. This design choice is implemented to compensate for human memory. It is always more beneficial to have the user recall rather than remember [14]. Recall refers to the type of memory which is very familiar; such as remembering your best friend's name. Remembering in the sense of user interface design is an active form of human memory; such as remembering a name of someone you just met. Although the medical images themselves are $2 \mathrm{D}$, they ultimately describe a 3D interpretation of the patient anatomy through mental transformations [16].

This simple design choice sounds obvious, but developers were presented with a myriad of other potential methods for displaying the patient anatomy. Maybe even a log of notes/instructions that were made during the planning stage would have been useful during surgery. Should they have chosen a better way to display more than one data set to show different vital structures in different parts of the body? Would it have been better to colour-code the venous system to outline important structures like the vertebral artery on top of the skeletal system during spine surgery? One may even think that since surgery is planned in a 3D space, perhaps a 3D model of the patient anatomy would be best used to assist for navigation. The number of variations and possibilities of design choices are endless. Knowing that there is no need to view the medical images differently because of a high-level understanding of the patient anatomy saves time for both the developers in the design process as well as the surgeons when operating the systems.

This belief is further backed by other studies. Users which were both clinicians and non-clinicians were asked to perform three different tasks while visualizing the patient anatomy in three different ways in a human factors study [17]. Users were asked to visualize patient anatomy using the standard CT scans, a multiplanar view, a 3D environment model, and an AR environment. The multiplanar view is where three slices (one in each $\mathrm{X}, \mathrm{Y}$, and $\mathrm{Z}$ plane) are displayed intersectional to one another.

After becoming accustomed to the different visualization methods, users were asked to rate which visualization methods were the most useful during the tasks. The three tasks were to determine the shortest possible distance on the surface to the tumor inside of the phantom, align the tool with the longest axis of the tumor and specify the trajectory which would result in the least damage to critical functional areas and white matter found in the diffuse tensor images (DTI). The results of the experiment showed that the clinicians were able to determine the solutions to the tasks faster in the standard CT scans as well as the AR environment. Overall, clinicians found the multiplanar view to be the least helpful [17]. 
Non-clinicians found that the AR environment was the best for visualization. In both groups, the multiplanar view was the least useful [17]. The belief that the standard 2D visualization was one of the most useful methods of understanding patient anatomy for the expert user base is reinforced by the findings in this study. It is interesting to also note that the AR view of the patient anatomy was shown to be the most effective for both the expert and non-expert groups.

Although commercially available surgical navigation systems do take some consideration in the guidelines for developing user interfaces, there are more that should be further addressed. Concerns for usability in surgical navigation systems include:

1) optimizing user interactions - Ensuring user input obtains the largest effect for the smallest input

2) maintaining the locus of control with the user - Ensure that the system can accomplish iterative small menial tasks instead of the user

3) human memory - Recalling (where the information is intuitive) vs. remembering (when the user needs to actively remember the information)

4) maintaining display inertia - Changing between screens/options should appear like one another so that the user does not become disoriented

5) accommodating users of different skill levels - User interfaces should be easy to use for both experts and novices

6) cognitive directness - Lessening the number of mental transformations/mental calculations

7) cognitive overload - Managing the amount of information at any given time to prevent the user from feeling overwhelmed

Optimizing user interactions in this case overlaps with maintaining the locus of control with the user. Optimizing user interactions refers to ensuring that the actions required from a user leans towards the most effect for the smallest effort, and generally making user interaction more efficient. Maintaining the locus of control with users means that users should feel as though the system is working for them rather than the user is inconvenienced by the system [14]. In the context of neurosurgical navigation, the requirement to manually input coordinates describing the surface of the patient's spine can feel like the user must work for the system for it to begin navigating during surgery. The sheer number of points required for registration is also a demonstration of poor efficiency and fails to optimize user interactions. Failure to meet both user development guidelines can result in frustration and contributes to the low adoption rate of neurosurgical navigation during spine surgery.

The limitation of human memory is another guideline that has not been met in navigation system workflow. As mentioned earlier, the user is shown the same medical images from treatment planning on 
the system during navigation. The trajectory of the tracked tool in view is shown superimposed directly onto the original familiar patient scans. Although the system works well in theory, it may not necessarily work in the context of the operating room. Some tools may not necessarily have a tracking array at all. In addition to this caveat, tools which are trackable for one system may not necessarily be trackable for another system. For example, a Medtronic IR tracked tool may not necessarily work with another spine navigation system from Stryker. In the event where the tracker is removable, surgeons have been mistaken in the past where a Medtronic tracking array was transferred to a Stryker tool. The navigation system mistakes the Stryker tool as the original Medtronic one and is no longer reliable as it incorrectly interprets the location of the tool tip, providing the surgeon with incorrect information and potentially leading to complications during the procedure. Considering the navigation system is only capable of recognizing the array and not rest of the physical tool, this is a particularly dangerous set up. There were no "warning messages" implemented at the time to state that the array is on the wrong tool to prevent the configuration since only the IR array is visible to the system [18]. Now, these systems are required to have additional steps to set up the system including tool verification to ensure that the correct tools are used. This disconnect between non-uniform systems may cause instances where the tool is untracked, and navigation is no longer available. For example, a system may have a tracked pedicle finder, but may not necessarily have a tracked screwdriver. When the surgeon uses the screwdriver, the planned trajectory of the screw may not be available. The workflow forces the surgeon to remember where the intended trajectory was on the screen and then recreate it from memory when deploying the pedicle screw. When comparing the view from the surgical site to the navigation screen, surgeons must fully understand the context and spatially relate the information shown from two sources [19][20][21].
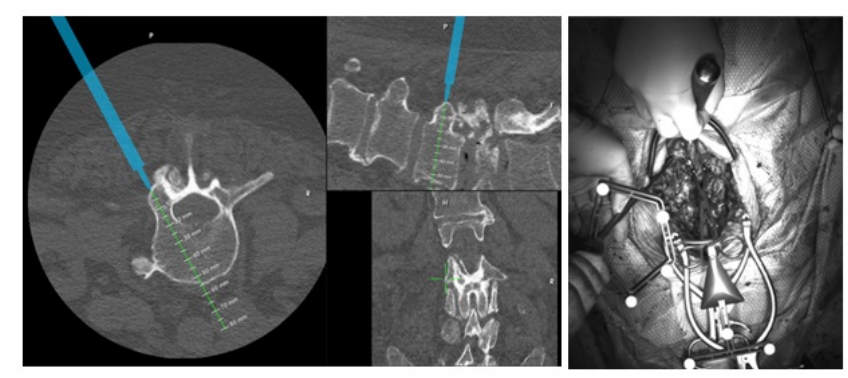

Figure 3: There is a visual disjunct between the surgical navigation screen (left) and the surgical site (right). The blue trajectory is the position of the tracked tool. The tool is the only piece of information linking these two views. 
Depending on how severe the deviation from the planned path is, the patient can potentially face further complications. Some consequences include acute neural and vascular failure, life threatening complications or additional surgery to correct the screw trajectory [9].

Maintaining display inertia is another useful design tool that is unfortunately often overlooked in navigation. When analyzing normal text menus, it is important to make subsequent screens in the sequence appear like the previous one in terms of style and formatting. If users must adjust between screens, it increases eye fatigue which can potentially reduce productivity and accuracy. By using a system for navigation, the additional computer screen causes the users to frequently shift their gaze from the surgical site to the screen. This changing line of sight is apparent when the user is checking that the intended trajectory is correct before remembering it later for a potentially untracked tool. Although this constant transition between the surgical site and the navigation screen may not appear to be a large nuisance, it is a very abrupt change in display inertia and can be the cause of deviating from the intended screw trajectory. In this back-and-forth checking process, surgeons can potentially move their tools on the submillimeter level by moving their heads without knowing. The irony is that although surgical navigation systems are capable of registration to the submillimeter level, the results from pedicle screw insertion are not necessarily equally as accurate because of the current workflow and set up in the operating room. Additionally, the screen can be oriented in awkward positions for multiple users, causing neck and muscle strain over time.

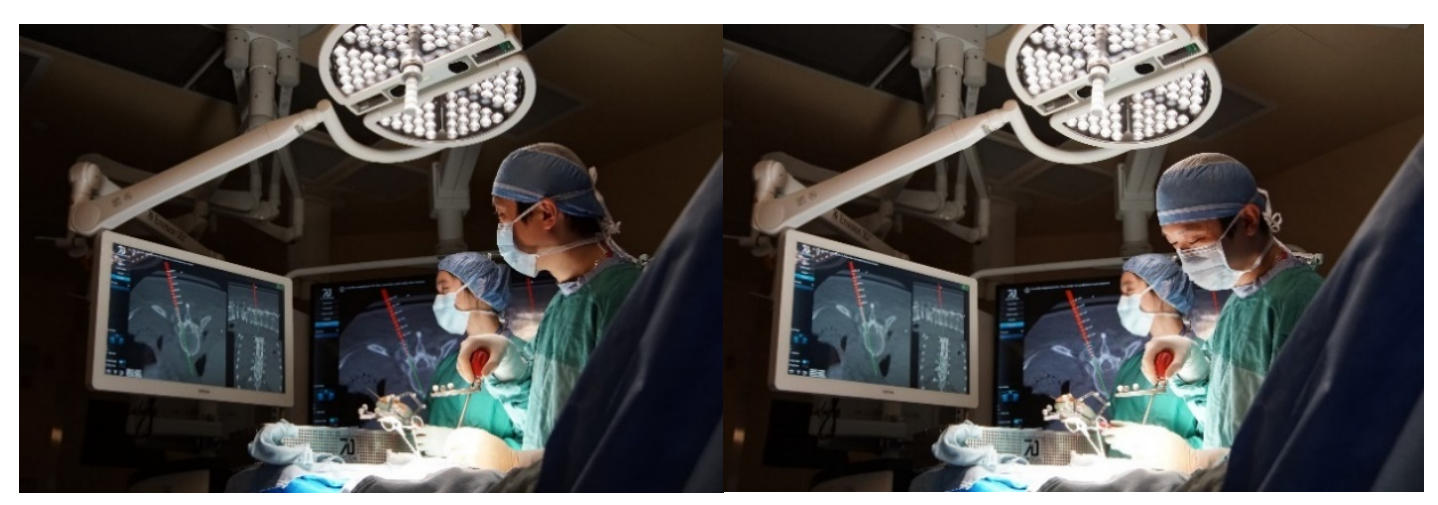

Figure 4: The workflow prevents surgeons from focusing on the surgical site. Surgeons must also keep their hands as still as possible to not lose the intended tool position. This constant changing view from the screen to the surgical site also disrupts display inertia. 
In this case, maintaining display inertia is an issue that coincides with another guideline: cognitive directness. By implementing cognitive directness, this means that the user interface should lessen the number of mental transformations for the user. In the case of navigation, users need to mentally transform the coordinate system from the medical images to the physical surgical site during surgery. This need for multiple mental transformations is another cause for the loss of display inertia. The trajectory of the tracked tool and how the user holds the tracked tool is the common link between the two reference frames. The trajectory shown on the screen is mirrored from the tool held in the surgical site. To ensure that the intended trajectory of the tool will be in the correct place, surgeons need to look up and down between the navigation screen and the surgical site. This visual disconnect where valuable information is shown in two places can potentially lead to breaches and other complications.

Cognitive overload refers to the state where there are multiple forms of information presented to the user to assist them with completing a specific task. In the efforts in providing as much information as possible, the user is then overwhelmed and takes resources away from the main task [22]. In surgery, the information presented in the surgical site and on the surgical navigation screen can be overwhelming. In addition to this information, further attention is required when viewing what is shown under the microscope, x-ray, neuromonitoring signals, anesthesiology readings, etc. With all of the information given to the surgeon and precautions they must take before proceeding with next steps of the surgery, it is easy to see why surgeons are subjected to cognitive overload while in the operating room [22].

The various shortcomings of commercially available surgical navigation systems make room for further research initiatives and propositions for better and more user-friendly systems. In the advent of new technologies and fast pace development, the possibilities of somewhat reinventing how humans interact with technology are endless. Ideas which were theorized decades ago are only coming into realization now because of improved processing and computing power to support them.

\subsubsection{Human Factors and Augmented Reality Navigation Systems}

The idea of using AR for medical purposes has been previously explored. Researchers in the 80s theorized that there would be multiple uses for AR in the medical world one day [23]. These ideas were unattainable at the time because the technology available was so premature. Only recently have AR devices, powerful cellphones, and Head Mounted Displays (HMDs) been accessible and cheap enough for the consumer level. 
AR in a clinical setting has been theorized for many purposes revolving the idea of visualization. There are certain landmarks which are visible to the surgeon by their own eyes after opening, but also many hidden structures that are only visible in CT or MR imaging [24]. By registering the patient specific scans to the surgical site, it augments the surgical field by displaying structures below the surface that would have otherwise been unavailable. This registration displays a pseudo "X-ray" vision effect for the user and can theoretically assist with surgical planning and navigation. Registering patient scans to the real physical patients is not only useful in a neurosurgical setting, but can assist practitioners with needle guidance, direct biopsy needles, show a 3D reconstruction of the developing fetus in a mother's womb, keyhole surgeries where patient anatomy is less exposed than open surgery, and many more medical applications [24][23].

A common concern regarding this topic is known as "inattentional blindness" [25]. Overlaying a virtual model of the patient on top of the surgical site risks blocking the surgeon's sight from seeing the real surgical site and can potentially add difficulty in depth perception [26][27]. Previous works have shown that using a mesh overlay has been more promising for the future of AR navigation as it allows for better context cues from the real world rather than a solid virtual overlay [28]. One reason why the mesh overlay is preferable is because it gives the user a sense of parallax or depth. Parallax is considered one of the most powerful methods of inferring depth to a user [17]. Other important cues that are useful for interpreting depth from 2D images include occlusion and stereopsis [29].

By creating an AR HMD for navigation, virtual content can be displayed directly on top of the surgical site while maintaining sterility. Using a computer screen to overlay a video see-through interpretation of the surgical site is an intermediary step, but ultimately still demands the user to make multiple mental transformations during use. An optical see-through HMD also allows for a more immersive AR environment which can be beneficial for the user to fully interpret virtual content more intuitively as an extension of the real world [23]. Optical see-through refers to the use of a clear OLED to display the virtual content on the real world. There are also a number of common head mounted devices in the operating room including surgical loupes and a headlight. Creating an AR HMD would not interrupt the surgical workflow more than the existing devices if designed correctly for the primary user base. 


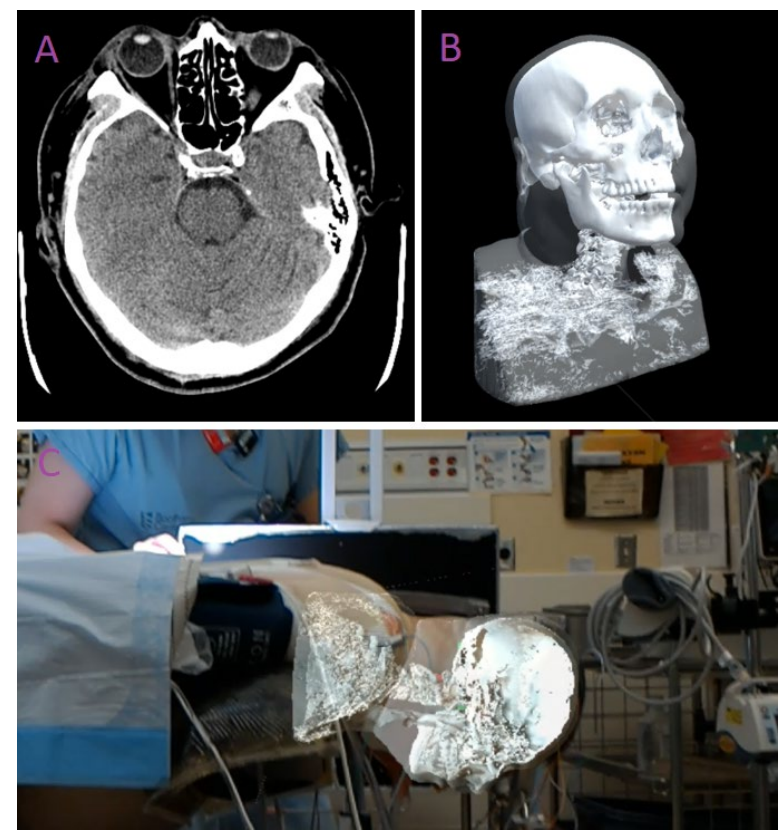

Figure 5: A) CT images are taken of the patient to diagnose and form a treatment plan. B) Based on the CT images, a 3D reconstruction can be made based on the grey levels for image segmentation. C) Using an AR HMD, the 3D model can be realized in the real world

In terms of the user developer guidelines mentioned earlier, there are several that can be addressed in an AR HMD navigation system. Immediately, we can see that the issues of cognitive directness and display inertia are already taken into consideration. By overlaying the medical images onto the surgical site, the number of mental transformations is reduced. All the information in both coordinate systems is in the same location without depending on the user to make the mental connection. In turn, display inertia is also reduced because there is no need to constantly change views between the surgical site and the navigation screen. Reducing the number of mental transformations and keeping all the information on the surgical site makes for a better user experience which conventional navigation systems currently do not have [14].

An AR navigation system also allows the developers to make a user interface which can accommodate users of different skill levels. It is known now that AR as a method of relaying instructions from one user to another has been shown to be faster and more efficient than other traditional methods such as instruction manuals [30] [31]. By removing the manufacturer representative from the operating room, surgeons also get the chance to learn how to operate the system themselves rather than depend on someone else. Reducing the number of people in the operating room also decreases the rate of infection [32]. Creating an interface which is more user-friendly lowers barriers for adoption in an operating room. 
Although not all usability issues are taken into consideration as easily, such as maintaining the locus of control with the user, optimizing user interactions, and minimizing cognitive overload, the development of an AR HMD for surgery gives developers a chance to redesign the entire workflow of surgical navigation devices. Given the feedback from surgeons who have used navigation before, developers are also given the chance to learn from previous technologies as a cautionary tale regarding user interfaces.

Although AR technologies in the operating room propose great promise and advancement for the future of surgical navigation, transitioning from one version of technology to another is very difficult. Since humans are likely to draw from real world analogies and previous experiences, ensuring a smooth transition for users to a new platform requires progressive updates to slowly shift usability habits. To suddenly develop an HMD for the operating room and expect users to immediately adopt a new system without considering operating room workflow, existing limitations, and an understanding of the personnel running the operations is to repeat the existing pitfalls of current surgical navigation. A slow, logical progression with introductions to new technologies is key for widespread adoption [14].

\subsubsection{State-of-the-Art Augmented Reality Systems in Neurosurgery}

At this current point in time, there is no existing stand-alone AR system for surgical navigation; however, there are a few systems which have AR capabilities that exist in conjunction with conventional navigation. Once again, these small introductions of AR to the operating room are important for a slow

integration and increase the likelihood for later adoption of an HMD system. Two systems which are both FDA approved with AR capabilities are the Scopus System from Stryker and an Augmented Reality Surgical Navigation (ARSN) System from Philips.

In both medical AR systems, the IR tracking system is the same, as well as the cumbersome registration process. The AR portion of the Scopus system is also a video see-through display for a laparoscope. A laparoscope is a camera which is specially built to see inside of patients during minimally invasive surgery (MIS). MIS refers to the type of surgery where much of the patient anatomy is not revealed like in open surgery. MIS is also referred to as keyhole surgery mentioned earlier [24] [33]. Surgeons use longer tools with smaller surgical sites to lessen the rate of infection in patients. Patients also experience less pain and faster healing times. However, MIS also takes longer than open surgery because many of the landmarks which are typically visible in open surgery, but that are now underneath the surface again. A laparoscope can be very useful in this scenario because of the lack of visibility compared that seen in open surgery. 
The Scopus system works based on presurgical planning. The user inputs the intended route to execute during surgery. After registration using tracked tools, the user can see the markings made in a 3D pathway during the planning stage. Using AR, the system creates various targets or visual landmarks for the user to follow as the surgeon makes their way to the desired area on the computer screen. Since the AR portion is overlaid onto a camera feed, this is known as video see-through AR. The AR system also displays the physical distance between the tool and virtual target in millimeters.
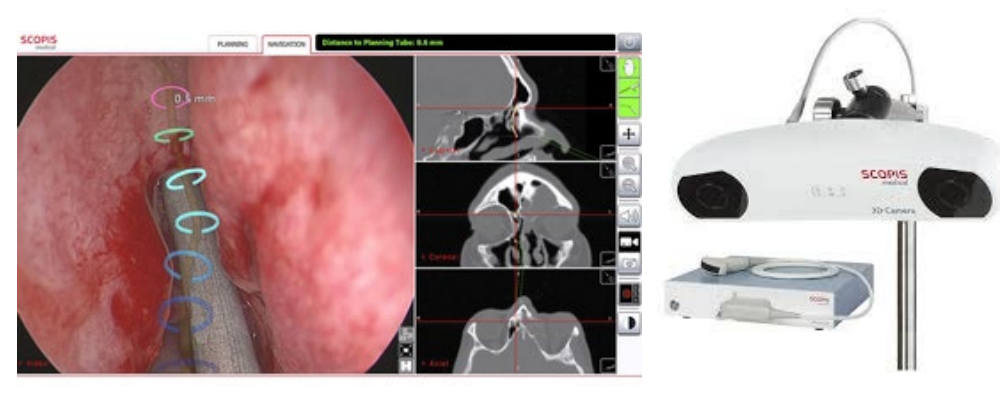

Figure 6: Scopus AR Navigation system uses a planned trajectory to create a path for guidance in conjunction with the conventional IR tracking system. The AR view on the left is also an example of the kind of view the surgeon has from using the laparoscope.

Another system which aims to imitate x-ray vision is from Philips - the Augmented Reality Surgical Navigation (ARSN) system [34]. ARSN is like other navigation systems where the trajectory is overlaid on top of the medical images as the tool moves with respect to the patient anatomy. The AR capabilities of this system are intended for spine procedures such as inserting pedicle screws. The same IR tracking technology is used to determine the orientation and direction of the tool based on the IR reflective markers. However, what makes this system interesting is the AR capabilities of the system. Using a physical marker system which is on the patient, the system can register an x-ray image directly onto the patient anatomy. The intended trajectory of the tracked tool is also placed directly on top of the x-ray [34]. The ARSN system also creates a video see-through AR environment. The cameras to provide the video feed of the surgical site is placed above the operating area on the $\mathrm{C}$-arm. This specific navigation system can take $\mathrm{x}$-ray images to give the surgeon a real-time update of how the anatomy has shifted. Since the both the camera feed and the x-ray device are positioned strategically, registration between the $\mathrm{x}$-ray image and the camera feed is simplified to a basic displacement based on the arrangement of each camera. 


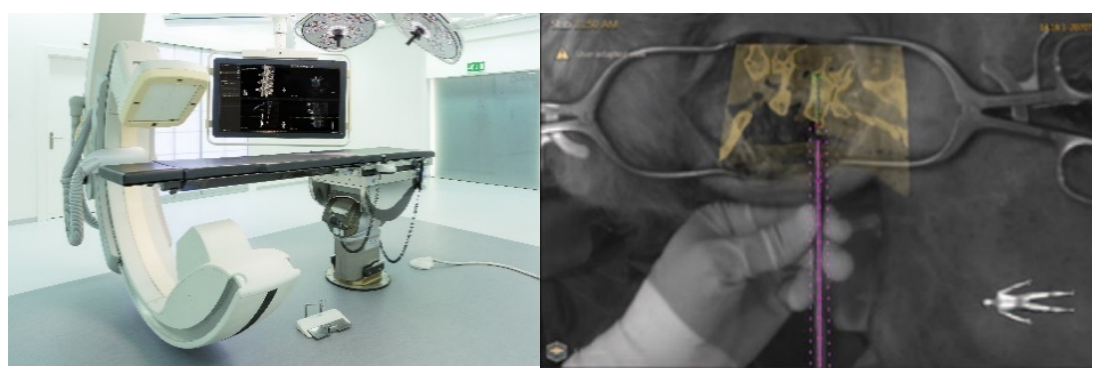

Figure 7: ARSN system from Philips. This system uses x-ray images and displays them on top of the surgical site provided a tracked tool is present.

In both $\mathrm{AR}$ navigation systems, using an $\mathrm{x}$-ray or $\mathrm{CT}$ is intuitive for surgeons to view as they are a common imaging modality. By choosing not to filter or alter the images in any way, the user also reads the $\mathrm{x}$-rays as they were trained throughout their education - the same benefit in the previous general of surgical navigation.

Improvements from the previously mentioned existing systems include less mental transformations and better efforts for maintaining the locus of control with the user. By virtually overlaying the x-ray directly on top of the patient anatomy, the user is not required to make as many mental transformations between the medical images and the surgical site. The internal patient scans are directly aligned with the physical patient anatomy, allowing for a simpler correlation between the intended trajectory and the physical insertion of the pedicle screw along the desired path. For both systems, the main navigation is still mainly carried out by conventional IR tracking systems found in previous non-AR assisted systems. The AR capabilities of these systems can be considered a visual aid to carry out the same procedure with clinical decisions primarily based on the tracking provided through the IR tracking system which has been proven to be accurate to the submillimeter level - unlike the AR implementations which are not published.

It appears that the AR views are meant to further inform surgeons through context, not to be used solely and separately from conventional navigation through IR systems. Enabling the user to view the surgical site through a different lens can be incredibly helpful in placing the information shown in the x-ray into context. The physical displacement between the view of the x-ray and the cameras is constant and only requires a fixed translation rather than previously requiring the surgeon to physically input the coordinates of the surgical site. The ARSN system also allows users to draw from existing analogies by displaying the surgical site on the computer screen as surgeons are currently conditioned to focus their gaze on the surgical site while operating. 
However, some drawbacks in terms of usability from the previous systems remain. The inability to provide cross-compatibility between tools is still an unmet need for complete patient safety. Although there are some trackable tools for the ARSN and Scopus systems, these tools are not necessarily transferrable to other manufacturer's pedicle screws, screw drivers, etc. The lack of cross compatibility between platforms potentially requires surgeons to rely on their human memory to recreate the intended trajectory. The ARSN system also requires users to look up and down, limiting the accuracy of the screw insertion due to the existing operating room workflow mentioned earlier. The AR mode from the ARSN system only uses a superimposed $\mathrm{x}$-ray image. The $\mathrm{x}$-ray image does not provide a 3D representation and is questionably accurate enough for navigation without the standard view with MRI images. Scopus uses the measurement system to compensate for the inability to show 3D information, but inherently does not propose 3D information for the user outside of the traditional navigation portion of the system. Given that current state-of-the-art systems without AR mode are accurate enough to the sub-millimeter, the AR mode is meant only for visualization of the trajectory to minimize the number of mental transformations, and does not necessarily show the same accuracy seen in older systems. AR mode is consistently an extension of the classic IR tracking system in these systems. Overall, we can see that there is a general desire to move towards the development of an AR navigation system, as expected from the literature.

\subsection{Thesis Motivation and Contributions}

There are three main contributions of this thesis. Overall, the components are linked through an understanding of HCI and lay the groundwork for the development of a functional and well adopted AR navigation system designed for the operating room. AR as an extension of conventional spine surgery has been known to be functional and assist surgeons when inserting pedicle screws or other hardware.

Our overall hypothesis is the following: Augmented reality can be deployed in the operating room through human factors considerations seen above in order to avoid the pitfall of slow adoption seen in neurosurgical navigation systems for spine surgery. For a higher likelihood for widespread adoption, we propose an evolutionary pathway for adoption seen below.

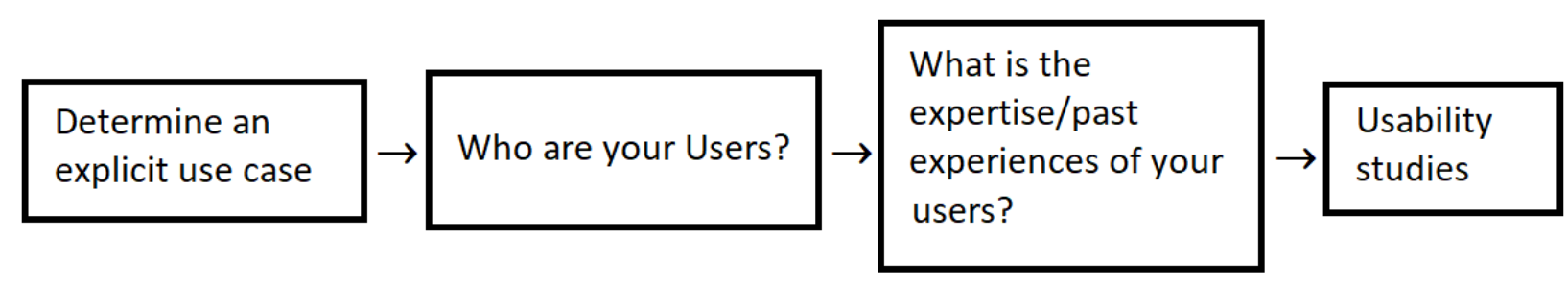


We determine that our use case is for spine surgery, and the users are neurosurgeons of varying skill levels. The last two portions of the pathway shown above are where the purpose of the thesis lies. Since our users are not guaranteed to have used navigation or AR, we propose a way to have a uniform experience through the first component of the thesis. The usability studies seen in the last part of the pathway are two-fold. Not only do we have to ensure that the users are compatible with augmented reality, but also ensure that the system works with the operating room workflow. The second and third component address both usability testing needs.

- In the first component of this thesis, the purpose is to further determine the efficacy of the AR extensions in existing surgical navigation and explore how these multiple mental transformations can lead to further error when depending on human memory.

- Second is an investigation to further understand the way surgeons perceive information to transition users towards an HMD as a mode for displaying information. Quantitatively understanding how an AR overlay should be displayed in the operating room can assist future developers in determining how information can be introduced without information overload and reduce cases of inattentional blindness.

- Finally, using the Microsoft HoloLens, we determine the accuracy of the virtual object placement of real patient anatomy while in the operating room environment. The overarching objective of this thesis is to determine how AR can benefit surgeons in the operating room and the logical progression for this technology to foster a beneficial relationship between surgeons and the future of AR navigated surgery.

\subsubsection{Guidance of Pedicle Screw Insertion Using AR Trajectories}

The lack of cross compatibility between tools and systems in the operating room in conjunction with the requirement to recreate screw trajectories without navigation are two very large flaws in the usability of existing CAN. Without cross platform compatibility, users can easily confuse tools and tracking arrays with the wrong tools. This issue not only produces unreliable tracking but can potentially cause the patient irreversible injury. Even when the correct tools are used with the correct systems, the workflow is suboptimal for the user. By requiring the user to remember the correct trajectory and potentially need to recreate it without navigation, there is a possibility for mis insertion which also causes damage. Both of these inconveniences in surgical navigation have the potential to drastically change the patient outcomes for the better or worse.

Virtually imposing lines from stereoscopic cameras which show the desired trajectory into a spine pedicle can help in accounting for human memory. Initially, the process for determining the correct trajectory is 
the same. Using a tracked tool, surgeons determine what the desired trajectory is. In an AR system, the trajectory could potentially be saved as a 3D line directly on the surgical site. Afterwards, aligning an untracked tool with the saved trajectory is much simpler than relying on the user to recreate it from memory. The second tool used does not need to be tracked to maintain navigation. Not only does this simple solution compensate for human memory and improve workflow, but it also potentially solves the issue of cross-compatibility. By superimposing a virtual trajectory to trace onto the real-world surgical site, navigation is maintained even when a tracked tool is not present in the surgical field.

\subsubsection{Surgeons and the perception of Augmented Reality}

$\mathrm{AR}$ as a stand-alone technology has presents an infinite amount of design choices in user interfaces now that the virtual media exceeds the boundaries of a computer screen. Looking at commercially available products, all have their own different interaction choices to cater for a wide audience; but how do surgeons interpret the virtual information in the real world? By creating 3D models from patient specific CT images, these models can be overlaid on top of the real patient anatomy. Users were asked to answer various questions about rendering the virtual model to see which choices were most appropriate for an AR navigation system and which were the most intuitively informative.

\subsubsection{AR Placement Accuracy}

AR technologies have come so far and have never been so advanced. AR itself has evolved and manifested itself into daily life as smartphone devices and HMDs. Although technologies such as the Microsoft HoloLens have some impressive capabilities, the question remains if existing HMDs are usable for surgical guidance. Given that there are also various methods of interacting with virtual objects, it is also questionable which method gives the greatest placement accuracy. By comparing the placement accuracy of three different manipulation methods, the most accurate method was determined. After, the same tests were completed in the operating room to determine if the same results were capable given the workflow restraints.

The rest of the thesis will be organized as the following:

- Chapter 2 will focus on the integration of AR features into an existing surgical navigation system. Virtually imposing a line directly onto the surgical field can improve the workflow for pedicle screw 
insertion through limiting the need to remember screw trajectories between changing tools. This application of AR can assist surgeons in maintaining the accuracy of navigation when only untracked tools are available.

- Chapter 3 will discuss usability tests to determine how surgeons best understand AR overlays when placed in the surgical site. Surgeons of various skill levels are interviewed while in the operating room and asked directed questions regarding ergonomics and other data available through the HMD. Understanding how surgeons take in information is key to developing a surgeon-centred HMD which can truly assist the main stakeholder during surgery.

- Chapter 4 further discusses a quantification method for virtual object placement relative to the real world for various manipulation methods. Using three main methods of interaction, we explore the most accurate method for registration and discuss the viability for clinical use.

- Chapter 5 finally gathers the main conclusions and findings of this thesis and discusses the next steps for this body of work. Given the foundation of human factors needs presented here, we provide the information required to develop the next generation neurosurgeon-centred HMD. 


\section{GUIDANCE OF PEDICLE SCREW INSERTION USING AUGMENTED REALITY TRAJECTORIES}

\subsection{Preface}

There are a multitude of reasons that AR can be useful as a tool in the operating room. Some of these include the better human computer interaction, a more optimal workflow integration, and higher likelihood of widespread adoption compared to existing navigation systems.

To abruptly introduce an HMD device into the OR is to repeat the same pitfalls that existing navigation devices have already faced - non-intuitive systems, or those designed which do not act in a similar manner to previous systems will discourage adoption. Adoption can most readily be achieved through a slow, evolutionary introduction of new technologies with minor modifications of existing systems to ease users into the idea of AR in the OR.

A great example of transitioning users (yielding positive and negative results) is the progression of the Windows operating system. From the first general-user based iteration of Windows, the desktop setup has stayed relatively the same from Windows 95 until Windows 7. The toolbar is by default placed at the bottom, and icons are on the "desktop" in manila folders. This Windows operating system was made to mimic a real-world desk that would normally have papers, documents, etc. organized on the user's desk. These were the motivations for designing the user-centric Windows operating system as we know it. Windows 8 , however, was modified so abruptly compared to Windows 7 and other previous iterations that users chose not to adopt the system. Some clear changes were in the decision to remove the usual start menu and introduce a 'tiled' appearance for multiple applications to match changes made to their mobile platform. As a result, in 2012, Microsoft sales dropped 21\% in their PC sales [35]. The market share of Windows 8 was less still less than 7\% in 2014, with Windows 7 composing over $47 \%$ long after Windows 8 was unveiled.

Notably, starting in Windows 8.1 and being refined in Windows 10 (released in 2015), further design modifications were made. The result is that Windows 10 appears to be more of a hybrid of Windows 7 and Windows 8. Although Microsoft could have decided to entirely revert to a primarily Windows 7styled operating system, it would not assist users which have already adapted to Windows 8 . The start menu was reintroduced in Windows 10 to give the feeling of a Windows 7 start menu, with the addition of tiled applications connected to it - a distinct design choice from Windows 8. As of January 2019, the Windows PC market share was composed of Windows 10 at $53.18 \%$, Windows 7 at $35.05 \%$, and 
Windows 8.1 at $6.67 \%$ [36]. The progression of the Windows operating system is an excellent example of user adoption on a very large scale, the necessity to create iterative and evolutionary designs to improve uptake, and can serve as an interesting case when considering the implementation of new interfaces.

When considering the state of surgical navigation, existing navigation for spine surgery appears to be the "Windows 8 " of its time. Users are unhappy with the lack of user-centred design and would rather see changes that are better adapted to existing operating room workflow. AR in surgical navigation has the potential to be an interesting hybrid to accommodate both user groups - surgeons who do use navigation on a regular basis, and surgeons who do not. One of the ways in which AR can be implemented steadily into the OR is to incorporate small aspects of AR into existing CAN systems, much like how Windows 10 contains features such as a "partially tiled start menu" from Windows 8 , but in the same format as the start menu in Windows 7. By ensuring the same set up and familiarization to existing navigation systems, users can slowly become more accustomed to using AR features for smaller scale implementations. One opportunity for a small AR feature during surgery is to assist users with pedicle screw insertion.

Pedicle screw insertion is an interesting use case for developing an AR feature. Although the introduction of existing CAN systems has already significantly reduced the number of misplaced pedicle screws, the workflow of these devices is still somewhat impractical. CAN systems rarely address issues their users may encounter and do not design interfaces that work to assist them. As it currently stands, users are often expected to remember the trajectory of the tracked tool and recreate the same exact position potentially without navigation if screwdrivers and drills are untracked - a somewhat regular occurrence. This process is completed in addition to the need to understand the different transforms between the surgical navigation information with the physical surgical site. AR extensions such as the ability to maintain a trajectory on the navigation screen are incredibly simple but effective solutions for multiple difficulties experienced in the existing operating room workflow. 

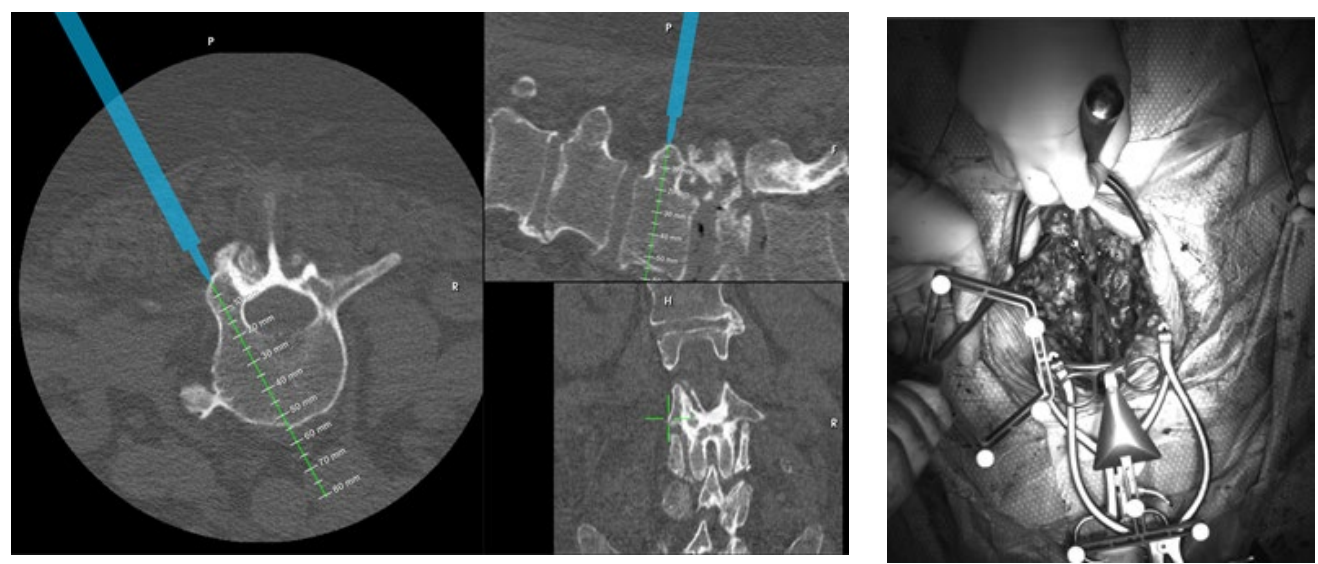

Figure 8: The image on the right displays the navigation screen of the 7D MvIGS system where the blue line is the tracked tool and the green line is the intended trajectory. On the right is the overhead view of a standard surgical site for spine. There is a disconnect in transferring the outlined trajectory to the surgical site when using an unnavigated tool where the view on the left pictured here is no longer available.

An AR extension which outlines the desired trajectory not only elicits the need to remember the intended path into the patient anatomy, but also assists users through lessening the number of mental transformations during surgery. By drawing a virtually superimposed line directly on top of the surgical site (using video see-through methods), the position of the tools is then explicitly aligned with the desired trajectory seen on the radiological images. Using the system to remember the intended trajectory for the surgeon decreases the cognitive load placed on the user. In addition to less cognitive load, users will now have the option to use tools which are not tracked as the trajectory is recorded for the user to follow. Now that the trajectory is saved, and navigation is maintained, users can use any tool to trace the trajectory if the correct length of pedicle screw is chosen to prevent breaching.

Although maintaining navigation while switching between tracked and untracked tools is incredibly helpful, there is a more frequent and frustrating cause for loss of navigation during surgery. To physically set up most navigation devices, the IR tracking cameras are placed on a tall metal stand so that it can view the surgical site from above as well as any tools which enter the system's tracking volume. When placing the IR cameras into position just before surgery, there are no visual cues for personnel to ensure that the tracking volume (or the stereoscopic IR camera viewing frustum) encompasses the entire surgical site. If the surgical site is at the edges of the tracking volume, it is very common for users to unexpectedly lose navigation during the surgery. Although these tracking systems do have aiming lasers which imply the centre of the tracking volume, they do not make a clear representation of the specific boundaries. If the surgical site is at the edges of the tracking volume, it is very common for users to unexpectedly lose navigation during the surgery. 


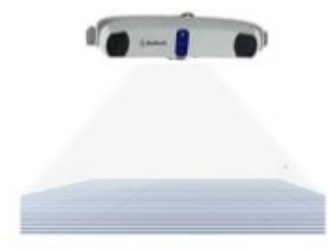

(A)

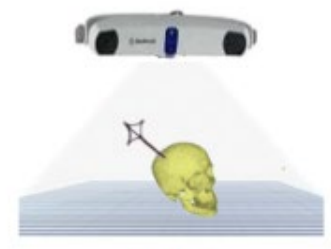

(B)

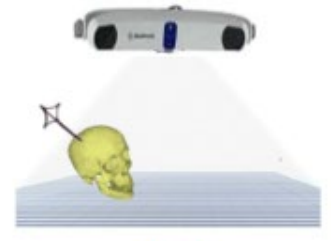

(C)

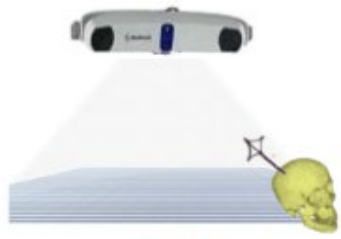

(D)

Figure 9: There are many possible causes for losing navigation regarding placement of the IR tracking system (A) The optimal spectrum is not visible to users because it is IR light. (B) Ideal navigation placement with respect to the surgical site (C) Non-optimal placement, the IR reflectors on the tool are outside the invisible range (D) Nonoptimal placement, the surgical site is out of range.

In addition to a difficult set up procedure, the operating room workflow itself can cause the system to lose navigation. The sheer number of personnel and other large equipment in motion throughout a surgery can potentially accidently move the IR tracking cameras (and subsequently the tracking volume). At times, personnel walking past the tracking cameras unaware of their function can also occlude the IR cameras' vision. Even the surgeon's own head or body can potentially block the view of the surgical site from IR cameras. Without any visible light cameras attached to the IR camera set up, it is very difficult and impractical to simply place it in an optimal path.

In the case of the 7D MvIGS System, one iterative solution is created through the integration of the IR tracking cameras into a surgical light head, reducing the likelihood of camera occlusion and improper alignment as surgeons will not block their own surgical light and the surgical light acts as a guide of sorts. While this improves workflow, this does not solve the problems of non-intuitive trajectory representation, or the problem of non-tracked tool guidance [37].

Drawing lines directly onto the surgical site to denote an ideal trajectory is a simple but effective solution for several inconveniences that surgeons face in the operating room. By making these small changes, there are multiple potential improvements manifested through human computer interaction, the operating room workflow, and cross compatibility between surgical navigation devices.

This study aims to maintain the precision and accuracy of surgical navigation when tracking is unavailable tools regardless of manufacturer, IR marker configuration, and in some cases, regardless of the IR camera position. Safe zones are displayed on top of the surgical site to preserve situational awareness, lessen cognitive overload, and assist in visualization of target trajectories with respect to 
patient anatomy in relation to medical imaging. Moreover, the integration of these trajectories on existing surgical navigation technology acts as an evolutionary step to improve future adoption of augmented reality systems.

\subsection{Methodology}

To ensure that this method for AR assistance in pedicle screw insertion is useful, we measure the extreme angles that mark the boundaries of the safe zones. There are four different ways of breaching the spinal column during surgery. The correct trajectory is the reference for each of these angle measurements. If the trajectory is too medial, there is a risk of breaching the spinal cord. Too lateral can potentially cause harm to surround organs such as the lungs and the heart. To be superior or inferior from the desired trajectory causes the breach of other vertebral bodies.

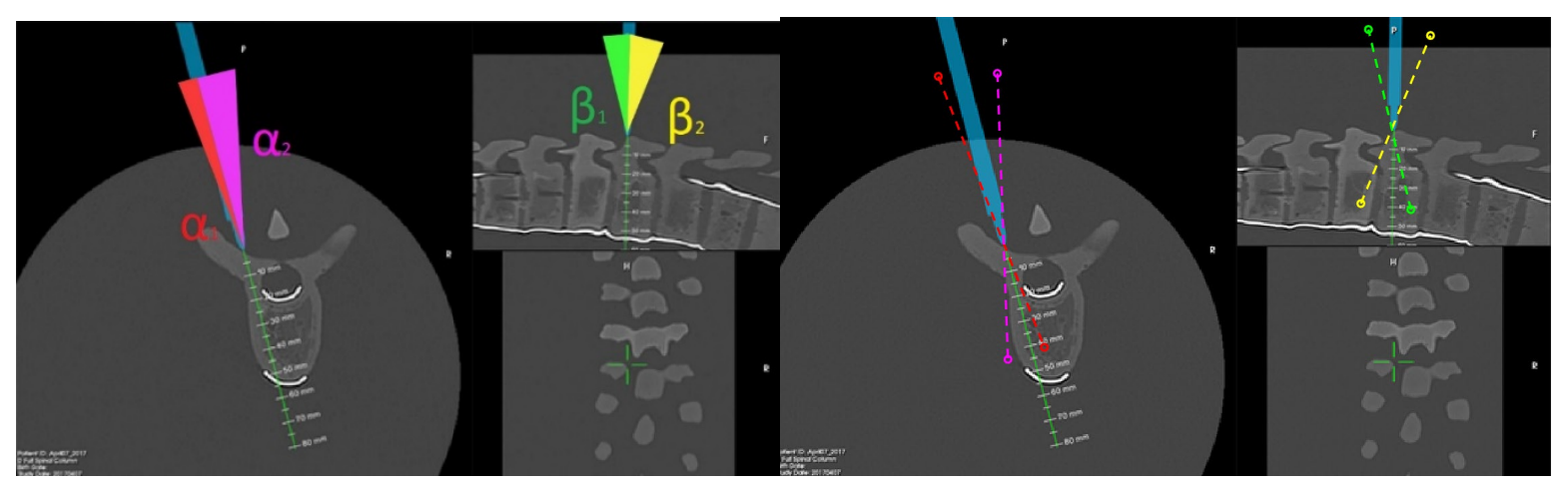

Figure 10: (Left) Angle Measurement system notation. The blue trajectories shown by the tracked tool are considered the reference trajectories. The dotted lines are used to describe different types of breaches. The red, pink, yellow and green lines represent medial, lateral, superior and inferior breaches respectively. (Right) $\alpha_{1}$ shows the angle made from a medial breach and $\alpha_{2}$ shows a lateral breach; these zones are noted by red and pink zones respectively. $\beta_{1}$ shows a superior breach and $\beta_{2}$ shows an inferior breach; these zones are green and yellow respectively. The lines differentiating in between these safe zones is considered the correct trajectory.

While the extremes of the safe zones are recorded, the same angles made on the surgical site are also recorded through stereoscopic cameras. The 7D surgical navigation system contains two cameras in the surgical light head attached to the system [figure $\mathrm{x}$ ]. This light head overlooks the surgical site allowing the two cameras to have a clear view. Considering the operating room light heads are normally about $90 \mathrm{~cm}$ above the surgical site, the cameras have a focal length of $90 \mathrm{~cm}$. Using two cameras are important for maintaining $3 \mathrm{D}$ vision. Like human vision, two eyes are needed to infer a disparity map which allows humans to interpret depth [38]. 


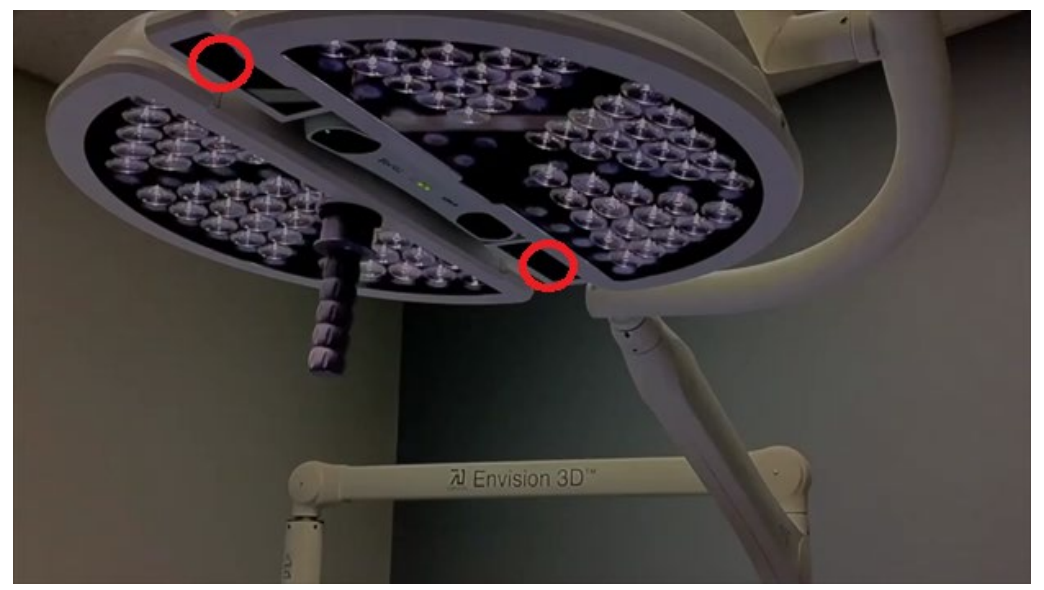

Figure 11: The light head of the 7D MvIGS system. Inside consists of the infrared tracker and the stereoscopic cameras. The location of the cameras is indicated by the red circles. This system is designed to overlook the surgical site to maintain the operating room workflow.

The view of the surgical site from above is useful for this study as it is a realistic representation of the information left when navigation is unavailable. Users are left with only the surgical site to recreate the intended trajectory.

Understanding the correlation between the surgical site and the navigation screen helps us determine why there is a visual disconnect between the two even though they are visualizing the same anatomy from the same patient from a different perspective. This data also helps us understand how accurate an overhead $\mathrm{AR}$ view is, and if it is suitable for use in the insertion of pedicle screws.

Using a spine phantom, this measurement process was completed on all possible spine pedicles from levels C2-S1 (second level of the cervical spine and the sacrum). Each trajectory requires 5 measurements (correct, medial, lateral, superior, inferior trajectories) for a total of 24 spine levels with a left and a right pedicle for each level. In total, 240 measurements were taken for the phantom portion of the experiment. When moving between spine levels, a new registration was completed to ensure the same level of accuracy throughout the measurement process.

After the phantom experiments were completed, the same measurement method was completed on 24 pedicle screws over the span of 5 patients. In total there were 120 measurements taken. 11 screws were inserted in the cervical spine, 6 in thoracic spine and 1 screw in the sacrum. The same process of reregistration was also completed in human patients to maintain accuracy throughout the surgery. 


\subsection{Results and Discussion}
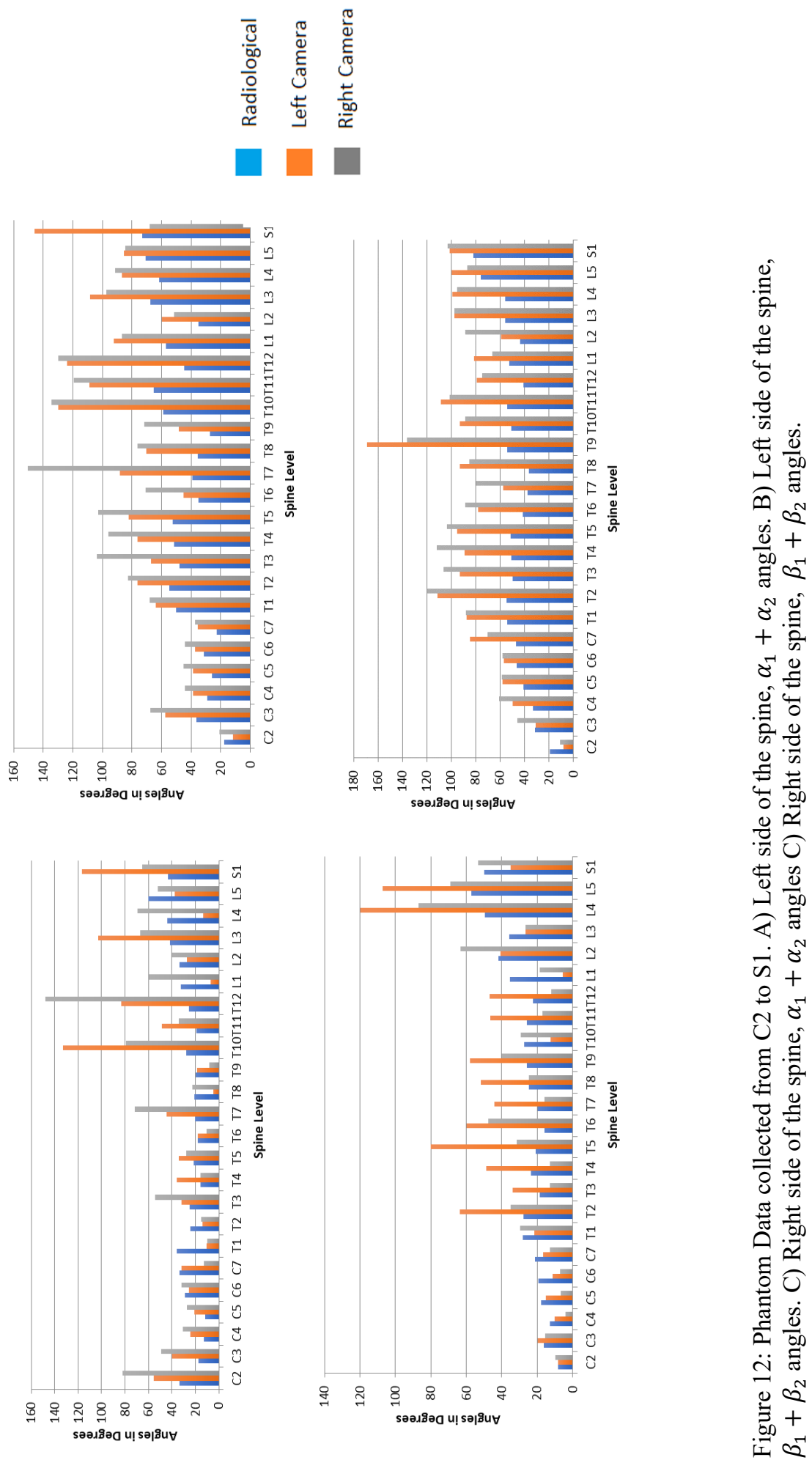

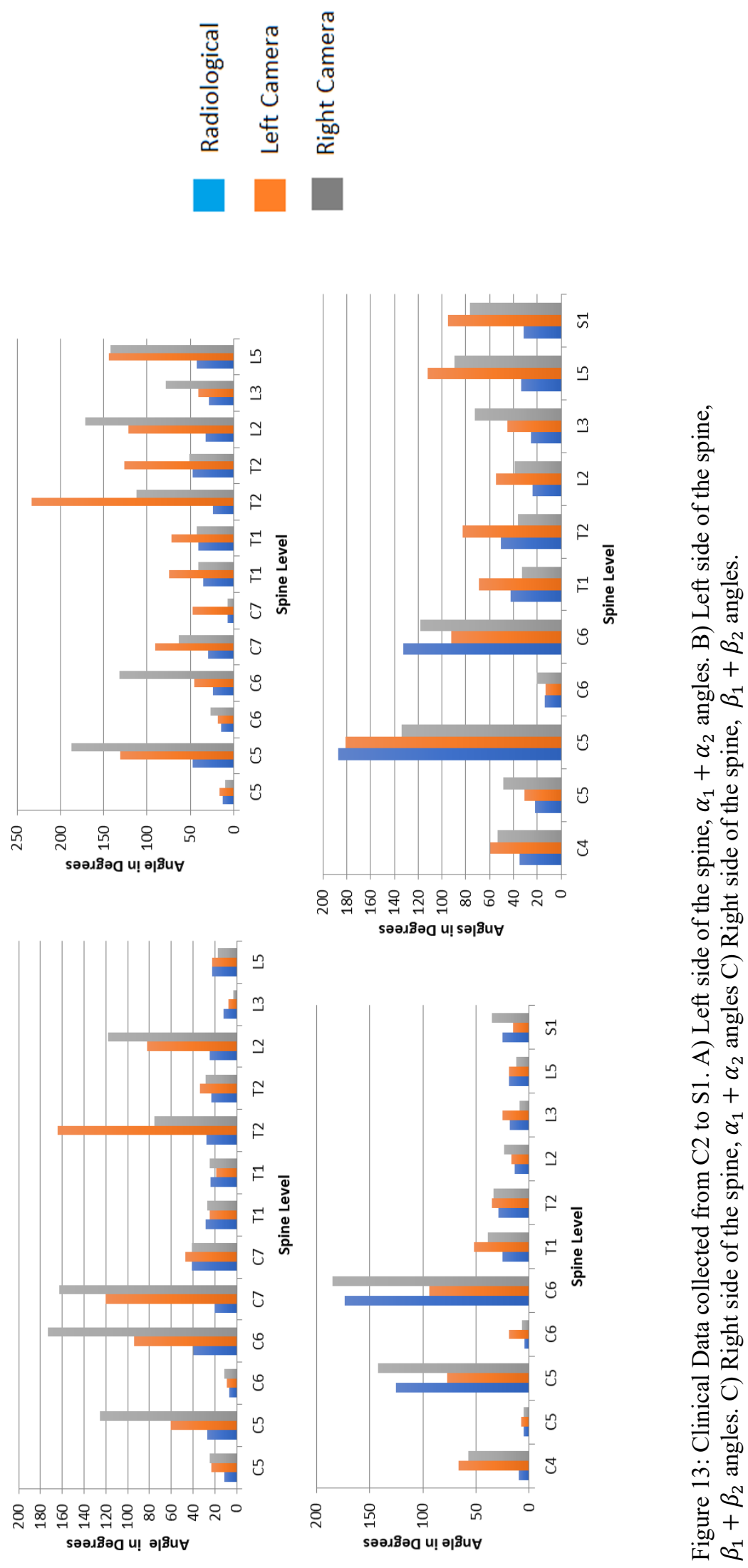


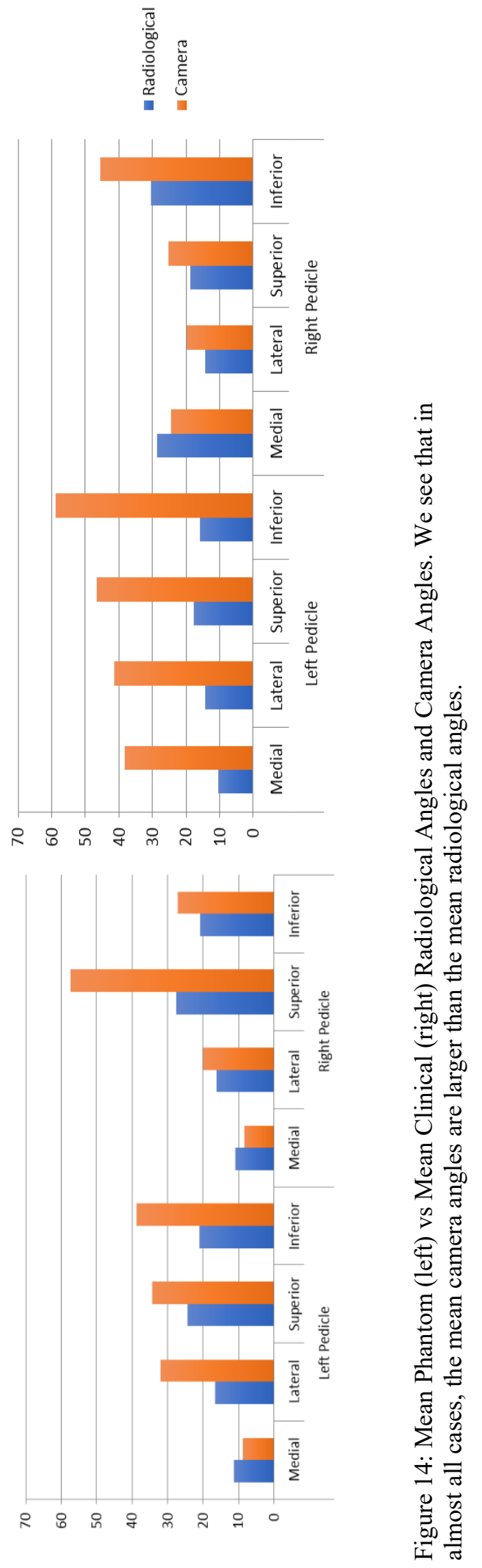




\begin{tabular}{|c|c|c|c|c|c|c|c|}
\hline $\begin{array}{c}\text { Spine } \\
\text { Side/Region }\end{array}$ & \multicolumn{4}{|c|}{ Left Side } & \multicolumn{3}{c|}{ Right Side } \\
\hline & $\begin{array}{c}\text { Average } \\
\text { Angles }\end{array}$ & $\begin{array}{c}\text { Left } \\
\text { Camera } \\
\left({ }^{\circ}\right)\end{array}$ & $\begin{array}{c}\text { Right } \\
\text { Camera } \\
\left({ }^{\circ}\right.\end{array}$ & $\begin{array}{c}\text { Radiological } \\
()^{\circ}\end{array}$ & $\begin{array}{c}\text { Left } \\
\text { Camera } \\
\left({ }^{\circ}\right.\end{array}$ & $\begin{array}{c}\text { Right } \\
\text { Camera } \\
\left({ }^{\circ}\right)\end{array}$ & $\begin{array}{c}\text { Radiological } \\
\left({ }^{\circ}\right)\end{array}$ \\
\hline $\begin{array}{c}\text { Cervical } \\
(\mathrm{n}=6)\end{array}$ & Alpha & $23 \pm 9$ & $33 \pm 12$ & $40 \pm 22$ & $16 \pm 4$ & $14 \pm 4$ & $9 \pm 4$ \\
\cline { 2 - 8 } & Beta & $27 \pm 6$ & $37 \pm 13$ & $43 \pm 14$ & $36 \pm 10$ & $48 \pm 24$ & $51 \pm 20$ \\
\hline $\begin{array}{c}\text { Thoracic } \\
(\mathrm{n}=12)\end{array}$ & Alpha & $23 \pm 5$ & $40 \pm 35$ & $41 \pm 40$ & $23 \pm 4$ & $47 \pm 18$ & $26 \pm 11$ \\
\cline { 2 - 8 } & Beta & $47 \pm 11$ & $82 \pm 26$ & $101 \pm 27$ & $48 \pm 7$ & $96 \pm 26$ & $100 \pm 17$ \\
\hline $\begin{array}{c}\text { Lumbar } \\
(\mathrm{n}=5)\end{array}$ & Alpha & $42 \pm 10$ & $37 \pm 34$ & $57 \pm 11$ & $44 \pm 8$ & $60 \pm 45$ & $53 \pm 26$ \\
\cline { 2 - 8 } & Beta & $58 \pm 13$ & $86 \pm 16$ & $82 \pm 16$ & $57 \pm 11$ & $87 \pm 16$ & $87 \pm 11$ \\
\hline $\begin{array}{c}\text { Sacral } \\
(\mathrm{n}=1)\end{array}$ & Alpha & 43 & 117 & 65 & 50 & 35 & 53 \\
\cline { 2 - 8 } & Beta & 73 & 146 & 68 & 82 & 101 & 103 \\
\hline
\end{tabular}

Table 1: Average magnitude of safe zones seen in the phantom tests. Average values were found for different regions of the spine (cervical, thoracic, lumbar, and sacral).

\begin{tabular}{|c|c|c|c|c|c|c|c|}
\hline $\begin{array}{c}\text { Spine } \\
\text { Side/Region }\end{array}$ & \multicolumn{3}{|c|}{ Left Side } & \multicolumn{3}{c|}{ Right Side } \\
\hline \multirow{2}{*}{$\begin{array}{c}\text { Average } \\
\text { Angles }\end{array}$} & $\begin{array}{c}\text { Left } \\
\text { Camera } \\
\left({ }^{\circ}\right)\end{array}$ & $\begin{array}{c}\text { Right } \\
\text { Camera } \\
\left({ }^{\circ}\right)\end{array}$ & $\begin{array}{c}\text { Radiological } \\
\left({ }^{\circ}\right)\end{array}$ & $\begin{array}{c}\text { Left } \\
\text { Camera } \\
\left({ }^{\circ}\right)\end{array}$ & $\begin{array}{c}\text { Right } \\
\text { Camera } \\
\left({ }^{\circ}\right)\end{array}$ & $\begin{array}{c}\text { Radiological } \\
\left({ }^{\circ}\right)\end{array}$ \\
\hline $\begin{array}{c}\text { Cervical } \\
(\mathrm{n}=6)\end{array}$ & Alpha & $24 \pm 13$ & $59 \pm 39$ & $90 \pm 66$ & $63 \pm 72$ & $53 \pm 34$ & $80 \pm 73$ \\
\cline { 2 - 8 } & Beta & $23 \pm 14$ & $58 \pm 41$ & $71 \pm 67$ & $78 \pm 70$ & $75 \pm 60$ & $75 \pm 43$ \\
\hline $\begin{array}{c}\text { Thoracic } \\
(\mathrm{n}=2)\end{array}$ & Alpha & $47 \pm 4$ & $76 \pm 7$ & $35 \pm 2$ & $27 \pm 2$ & $44 \pm 8$ & $37 \pm 3$ \\
\cline { 2 - 8 } & Beta & $37 \pm 9$ & $127 \pm 65$ & $62 \pm 30$ & $47 \pm 4$ & $76 \pm 7$ & $35 \pm 2$ \\
\hline $\begin{array}{c}\text { Lumbar } \\
(\mathrm{n}=3)\end{array}$ & Alpha & $20 \pm 6$ & $38 \pm 32$ & $46 \pm 51$ & $17 \pm 2$ & $20 \pm 3$ & $15 \pm 6$ \\
\cline { 2 - 8 } & Beta & $34 \pm 5$ & $103 \pm 38$ & $131 \pm 34$ & $28 \pm 4$ & $71 \pm 30$ & $67 \pm 21$ \\
\hline $\begin{array}{c}\text { Sacral } \\
(\mathrm{n}=1)\end{array}$ & Alpha & $\mathrm{n} / \mathrm{a}$ & $\mathrm{n} / \mathrm{a}$ & $\mathrm{n} / \mathrm{a}$ & $\mathrm{n} / \mathrm{a}$ & $\mathrm{n} / \mathrm{a}$ & $\mathrm{n} / \mathrm{a}$ \\
\cline { 2 - 8 } & Beta & 25 & 15 & 35 & 31 & 95 & 77 \\
\hline
\end{tabular}

Table 2: Average magnitude of safe zones seen in the clinical tests. Average values were found for different regions of the spine (cervical, thoracic, lumbar, and sacral). 


\subsubsection{Statistical Analysis}

When viewing Figures 12-14, we see that the angles viewed by the overhead cameras are relatively large in comparison to the radiological angles. This observation is further confirmed by the ANOVA and Tukey tests. The mean angles were as follows: left camera angles were $45^{\circ} \pm 48^{\circ}$, right camera angles were $48^{\circ}$ $\pm 52^{\circ}$, and the radiological angles were $137^{\circ} \pm 8^{\circ}$, with statistically significant differences in ANOVA testing $(\mathrm{p}=0.05)$. Following ANOVA, Tukey testing shows that the average differences between the two cameras is negligible and the differences between the radiological angles and each of the camera angles groups is statistically significant.

This magnification effect between the surgical site and the radiological view of the spine is interesting as it changes the surgeon's perspective between the surgical site and the CT images. It is even more understandable how screws can be improperly despite using surgical navigation. This change in angle size between the two views further reinforces the visual disconnect mentioned earlier - a larger deviation from the planned trajectory in the camera views reflects a smaller deviation in the radiological view.

In Tables 1 and 2, the average values for the different regions of the spine are shown for phantom and clinical testing respectively. Objectively, the levels of the cervical spine are the smallest vertebrae in the entire human spine. Naturally, the pedicles of these spine are also much smaller which results in smaller safe zones. Using the radiological view as a reference is useful in this case as they are the basis for presurgical planning. For the phantom cervical, thoracic, lumbar, and sacral spine, the averages were found to be $40^{\circ} \pm 22^{\circ}, 41^{\circ} \pm 40^{\circ}, 57.46^{\circ} \pm 11^{\circ}, 65^{\circ}$, respectively, for the alpha angles. For the corresponding beta angles, they were $43^{\circ} \pm 14^{\circ}, 101^{\circ} \pm 27^{\circ}, 82^{\circ} \pm 16^{\circ}$ and $68^{\circ}$. The differences between the average sized safe zones are consistently increasing in size for both axial and sagittal views as the vertebrae also increase in size. Using the phantom radiological angles as a reference for further safe zone measurement is also convenient as it also describes an ideal spine.

Similarly, when we observe the phantom camera views, the same trend is apparent. The left camera angles found for the phantom cervical, thoracic, lumbar, and sacral spine were $23^{\circ} \pm 9^{\circ}, 23^{\circ} \pm 5^{\circ}, 42^{\circ} \pm$ $10^{\circ}$, and $43^{\circ}$, respectively, for the alpha angles. The corresponding beta angles from the overhead left camera were found to be $27^{\circ} \pm 6^{\circ}, 47^{\circ} \pm 11^{\circ}, 58^{\circ} \pm 13^{\circ}$, and $73^{\circ}$ which also confirms our observations of increasing the size of safe zones with an increased size vertebrae size. The same trend is visible in the right overhead camera as seen in Table 1 and further confirmed by the statistically insignificant difference between the left and right overhead cameras proven from the ANOVA and Tukey tests $(\mathrm{p}=0.05)$.

Another observation is seen in the disparity of the cameras shown in the difference between the two cameras in the surgical light head. When viewing the left side of the spine, the left camera shows slightly 
larger angles compare to the right camera. The same is true vice versa when viewing the right side of the spine with respect to the right camera. This small compensation of the camera angles is due to the position of the cameras with respect to the patient anatomy and is cancelled by the disparity of the opposite camera. When one camera angle appears marginally larger, the other camera is marginally smaller. Using two camera views to follow a single trajectory is incredibly important to convey the true trajectory of the untracked tool on a computer screen. Despite this compensation effect, we still see that the average camera angles (between the left and right cameras) is still larger than the average radiological angles shown in Figure 7.

In comparison to the clinical data, it is important to note that the number of measurements is smaller considering that some levels which require spinal fusion are less common than others. In the case of the phantom data, it is easy to obtain data for every single level of the spine on both left and right sides. Clinical data is not as simple to collect the same volume of data as it is very rare for a single patient to require a fusion for every level of the

Many of the observations seen in the phantom data is also applicable in the clinical data. The trend of increasing safe zone size is still apparent as the spine vertebrae are also physically becoming larger. When observing the difference between the left and right cameras in the clinical data, the compensation effect is much more visible and potentially more pronounced compared to the phantom data. Since the position of the surgical light head can change from surgery to surgery, the compensation effect is much more apparent in comparison to the phantom data. While collecting phantom data, the phantom was moved minimally and only during reregistration for each level of the spine.

\subsubsection{Practical Interpretation and Limitations of the Study}

In each of the clinical cases, the location of the surgical navigation device changes within the room which is normal during set up for these devices. The infrared tracker is situated in the operating room with respect to the surgical site such that it is least likely to obscure the view of the infrared markers on the tracked tools. Since it also operates as an overhead operating room light, it is almost always able to see the surgical site with the least number of possible barriers. There is rarely any obstruction between the surgical light heads and the surgical site.

In the case of the 7D Surgical MvIGS system, the placement of the overhead infrared tracker also governs the placement and orientation of the visual cameras used for measuring angles in this study. Situating the visual cameras inside of the light head also ensures a clear view of the surgical site. Since there is no 
guarantee that this position of the cameras is consistent throughout the data collection in various cases, some angles can appear to be more exaggerated than others despite being on the same spine level. This is further confirmed due to the inverse relationship between the cameras in the clinical data. If there is a case where the left camera has a very large angle in the camera view, the right camera angle will be very small.

In the case of the phantom experiments, Figures 1-4 consistently show that angles for screw insertions on the left pedicle of the vertebral body observed by the right camera in the MvIGS system are larger than radiological views. The same is true vice versa when observing the right pedicle insertions through the left camera view. To limit the rotational error of the surgical navigation head, surgeons align one of the baseline axes between the cameras to be more in line to the patient's spine. Errors such as those found translationally are resolved using aiming lasers found in the light head to register to the correct level of the spine and ensure that the light head is level enough for registration.

Another limitation of this study includes the use of two cameras to fully display the depth data to show that all the information is acceptable for pedicle screw insertion. There are certain scenarios where this method is unreliable for use in guidance. For example, with patients experiencing scoliosis, the light head may appear to be in different positions than normal due to the abnormal curvatures of the spine. Changes in perspective can easily show distortion in the stereo camera view, i.e. cause some tool tips to appear longer and some other features to be shorter due to vanishing points [4].

Although the two cameras provide enough depth information, it can potentially be confusing and cause error since the information is spread over two views. Another cause for misleading information from the overhead view includes angles which move along the same axes perpendicular to the image plane of the cameras. These two may appear to look the same on the overhead view, but radiologically, those angles can be very different. The camera angles alone are not always enough to make safe insertions without the use of the IR camera tracking system. 

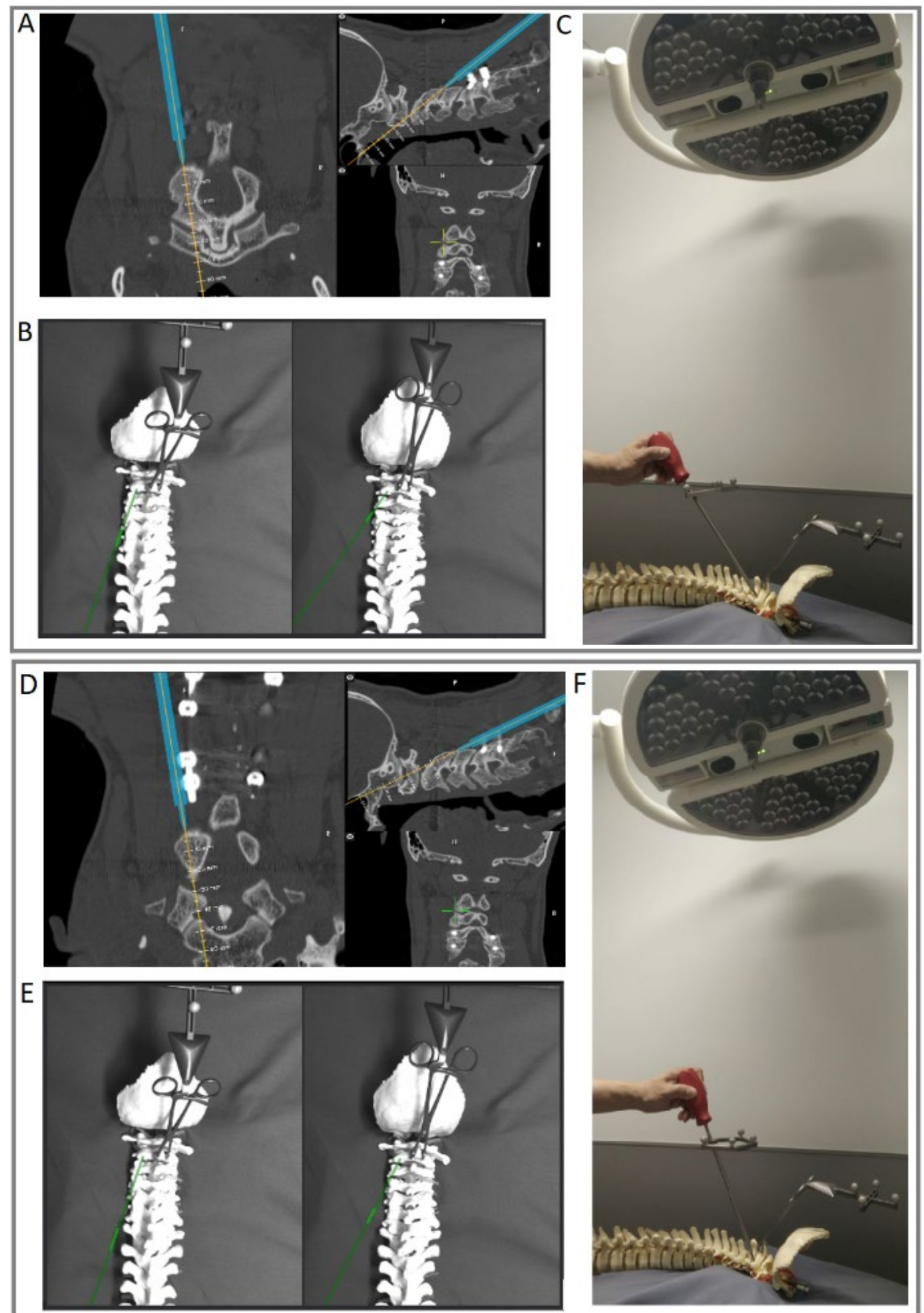

Figure 15: A, B, and C describe different views of a correct trajectory. D, E, and F describe different views of a superior breach. In both cases, the light head cameras are oriented parallel to the spine and the tool moves along this plane between trajectories. When comparing B and E, we see that the overhead cameras appear similar. However, the radiological angles seen in A and D show a difference of $13^{\circ}$ in the sagittal plane. $13^{\circ}$ is more than enough of a deviation to potentially cause a breach. 
Other causes for visual distortions are also dependent on the way which surgeons will be holding their tools. If the tracked tool is held in such a way that the tool appears to be relatively perpendicular to the surgical site, saving the trajectory for this position may be less helpful. The perpendicular tool position may occlude the tool shaft in a single view and therefore, only offer 2D information. Information presented in 2D is no longer reliable or useful for pedicle screw insertion. From Figure 15, in images C and F, we see that the surgeon's hand is positioned directly above the tool shaft potentially occluding the view of the site and the tool from the camera view.

As can be observed, the use of an AR overlay for display of surgical trajectories does seem to provide a safe enough margin of movement to allow for the safe insertion of pedicle screws based on trajectories taken from tracked tools. By implementing the display of AR trajectories onto the surgical site, the navigation system sees improved levels of display inertia between the surgical site and CT information, a decreased dependence on human memory, and further addresses issues such as cognitive directness. Moreover, this AR overlay acts to augment current surgical navigation standards, allowing users a more gradual, evolutionary transition into AR concepts, and making them more comfortable with augmented reality overlays. The use of AR overlaid trajectories serves as one of the initial ways to the slow introductory pathway seen earlier. Users reach a common experience with both AR and navigation after successfully implementing this system. As these overlays shift from video-see-through displays to Headmounted-displays, users will already be more attuned to the concept of AR overlays for trajectories, thus allowing for potential improvements to overall adoption. However, the limitations of these overlays do still exist, as demonstrated by the extreme cases listed above. Further, these limitations would likely exist for AR overlays in HMDs as with video-see-through; though, with knowledge of these limitations, surgeons will also be more likely to notice these pitfalls even when transitioning to future HMD-AR systems. 


\section{PERCEPTION OF AUGMENTED REALITY IN THE OPERATING ROOM}

\subsection{Preface}

As previously discussed, there is much impetus for deploying augmented reality head mounted display (AR-HMD) devices in the operating room (OR). A large motivation is for minimally invasive surgery (MIS) - also known as keyhole surgery. The objective for MIS is to complete procedures using small incisions in the patient, resulting in a smaller surgical site for surgeons to carry out their operations. A smaller surgical site also requires surgeons to use another set of tools that are longer to maintain a large reach within the patient body. In comparison, more traditional open surgery results in larger surgical sites to provide access to the operative anatomy. This procedure allows surgeons to see other physical landmarks underneath the surface of the patient which assists them to properly navigate the human body.

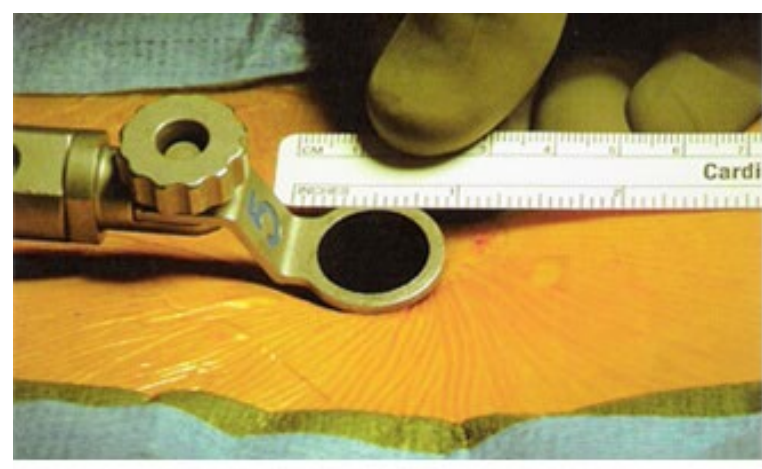

Minimally Invasive Spine Surgery

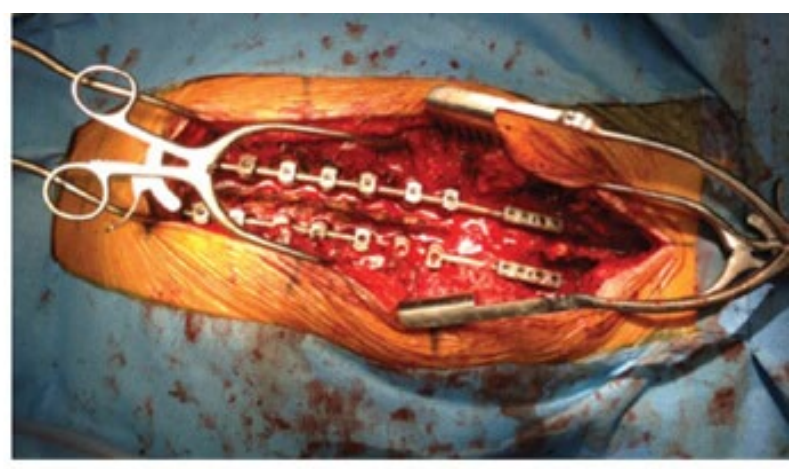

Traditional Open Spine Surgery

Figure 16: Comparison between minimally invasive spine surgery (left) and open spine surgery (right). The opening for MIS is only about $2.5 \mathrm{~cm}$ in comparison to the large surgical site created from open spine surgery [39].

MIS is commonly thought of as superior to open surgery because of lower infection rates, shorter recovery times, and lower pain medication dosages [40]. Although MIS has many benefits to the patients, it will sometimes add hours of time to surgery time because of the increased complexity. The smaller surgical site does not show as many physical landmarks in the patient's body which can make it more difficult to inform surgeons where in the human body they are. By overlaying the virtual patient anatomy on top of the real patient anatomy during MIS surgery to create a mixed reality view, surgeons would be able to see beneath the surface without making additional incisions. Ideally, an AR-HMD would have the capability to show the informative visual aids provided by open surgery combined with the quality of patient care from MIS. The ability to view underlying patient anatomy without additional intraoperative 
would also decrease the amount of radiation from x-rays used during surgery to confirm anatomical location [41].

Assuming a good registration between the virtual patient anatomy and the real patient anatomy, there are still several human factors to consider at this stage. Given the recent surge in AR-HMDs commercially available, there are a variety of different displays and rendering methods available. Most existing displays are directed for a general commercial market for consumers interested in home entertainment. Once again, using the mantra of user-centered design, it is important to specifically consider neurosurgeons as the primary user group. An intuitive AR-HMD deployed specifically for surgery has the potential to reduce the time surgeons need for MIS procedures, which, in turn, reduces the associated costs for running an operating room, as well as potentially improves patient outcomes. This section is directed towards determining the most intuitive methods for displaying virtual information in a surgical AR environment, as well as the ideal ergonomics that would inform the design of such a system.
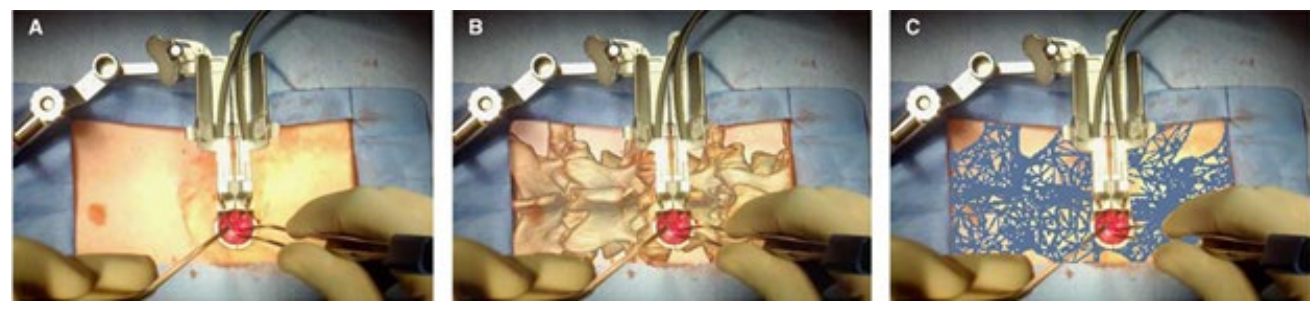

Figure 17: A) A standard surgical site for MIS. We can see that the site is very small and there are very few visual cues informing the surgeon where they are in relation to patient anatomy. B) Overlaying the virtual information from a CT scan allows surgeons to see where in the spine they are operating on. The mixed reality view allows surgeons to see within the patient body without a large surgical site C) There are numerous rendering methods available today to determine how best to show the virtual information without risking inattentional blindness, including wireframe methods visualized here.

Outside of the operating room, using AR or VR (Virtual Reality) as a tool for teaching and explaining has been both researched and commercialized to varying degrees. In comparison to standard medical imaging, patients who have neither a medical or scientific background appreciate AR and VR tools to more intuitively understand the various scenarios. Medical imaging can be difficult to interpret without the necessary training and practice that others (radiologists, surgeons, etc.) have from experience. Surgical Theatre LLC. is a company which has a pipeline of displaying patient specific anatomy onto a VR-HMD (Virtual Reality Head Mounted Display) for physician use including explaining surgical procedures to patients and trainees, as well as general patient education. Using multiple VR-HMDs also allows for a collaborative effort between surgeons of various skill level and surgical planning [42]. The ability to 
display information such that both highly trained individuals as well as untrained individuals can understand what they are viewing provides an interesting opportunity and can potentially expand the anticipated use case.

Several research groups have considered the same question regarding further understanding surgeons and the way that they process visual information. The most commonly used platform for usability tests is the Microsoft HoloLens due to its ability for rapid prototyping and spatial mapping [43]. In many cases, displaying the virtual objects such that they mimic real world objects has shown to be effective in interpreting a variety of concepts. One challenge seen in many AR environments is depth on a 2D screen through the addition of shading the virtual object [17][44]. This proposition of drawing from real world analogies and past experiences comes as no surprise as it is commonly employed in the field of human computer interaction [14].

An interesting phenomenon regarding the shading of the virtual objects is that too much shading is undesirable. There are two types of shading described - volumetric cel-shading and volumetric gradient shading. Volumetric cel-shading is described as a non-realistic form of shading where the edges of the anatomy are outlined in a dark color while volumetric gradient-shading is a more realistic form where the edges are gradually darkened. Abhari et al. found that $75 \%$ of the maximum value of the gradient-shaded and $50 \%$ of the maximum value is cel-shaded was the most effective ratio and combination for delineating edges of complex structures (specifically, an arteriovenous malformation- an abnormal blood vessel structure which has a higher likelihood to rupture compared to normal networks). Greater than 50\% cel-shading produced an emphasis on noisy portions of the complex anatomy which was undesirable [44][17]. Both qualitative and quantitative data showed that the participants found the shading to be helpful when asked if two blood vessel segments were continuous or not. These works also found that the stereoscopic view of the anatomy further increased depth perception compared to viewing a regular $2 \mathrm{D}$ image of the arteriovenous malformation.

Transparency is another property which can change the AR experience. Transparency is highly linked to inattentional blindness in many studies and is often a major concern with anatomical overlays during surgery. Inattentional blindness refers to the inability to find an unexpected object when the user is preoccupied by the current task at hand [45]. The main concern with AR during surgery is that the overlay can potentially prevent surgeons from locating unexpected findings which may not have been discernable during the presurgical planning stage. When observing the effects of inattentional blindness, the transparency is usually shown in these studies as three different manifestations being fully opaque, using no overlay at all, and a wireframe rendition of the virtual object to serve as a middle ground [46]. The wire frame is a version of the virtual model where the triangles that make the surfaces of the 3D object are 
outlined. The area within these triangles is transparent, making the real world visible through the triangles.

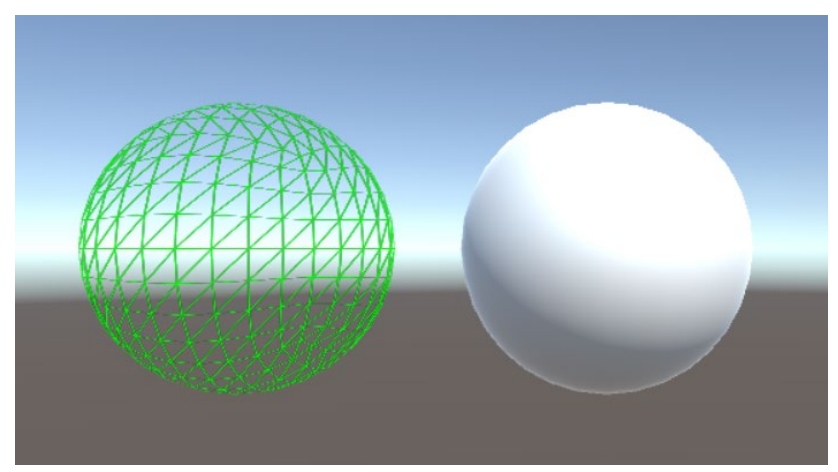

Figure 18: The sphere on the left is an example of a wireframe model. Notice that the triangles composing the mesh are outlined in green, but the internal area of the triangles are transparent. The sphere on the right is opaque, no triangles are visible and there is no transparency effect.

Experiments performed by Hallett et. al were aimed at determining if surgeons can locate the unexpected findings (either a suture or swab in the surgical site) in the patient anatomy. Inattentional blindness was measured by their ability to find these artifacts during the main task. To induce a direct increase of cognitive load, surgeons may have been given an additional task to complete in addition to the main task. Overall, surgeons performed better with the wire frame overlay in terms of inattentional blindness in contrast to an opaque overlay. There was no statistical difference between the wireframe and the control groups (the control in this case was no overlay used at all). The wireframe shows information regarding structures beneath the surface of the surgical site while allowing them to maintain focus on the primary task [45][47]. Understanding that a wireframe model can display both the virtual component and realworld components clearly is beneficial in terms of inattentional blindness. This study is further aligned with the results found in a meta-analysis regarding pilots and the use of a HUD (Heads Up Display) during flight. In the context of aviation, the display shows minimal information, with most of the display being transparent to allow the pilot to maintain view of the path. The HUD was shown to have little or no effect on inattentional blindness with the exception of a runway incursion event (i.e. an unauthorized aircraft, vehicle, or person is on the runway) during final approach (where the plane is descending for landing) [48][46]. 


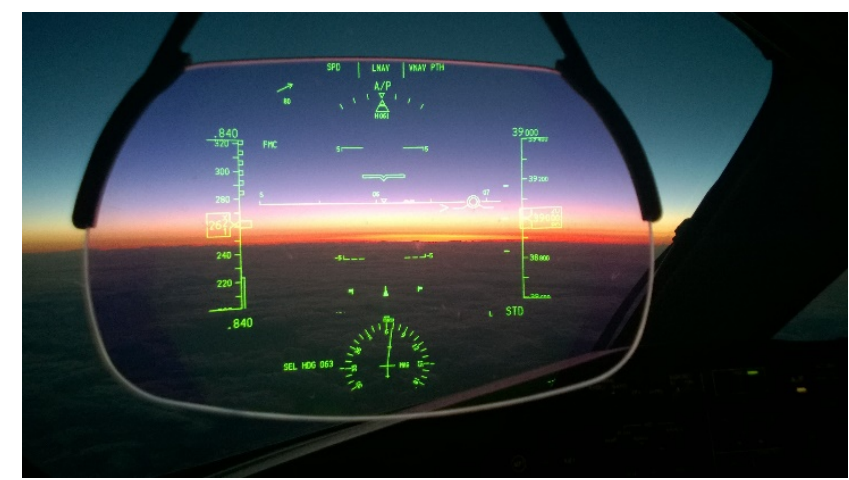

Figure 19: An example of a HUD for a pilot where minimal information is displayed while maintaining a clear view of the skies.

It is unclear at what the exact level of transparency is the most optimal for surgeons considering transparency is a spectrum and not simply three categories. When considering the design guidelines in $\mathrm{HCI}$, it does not make sense to provide a minimal representation of the patient anatomy onto the surgical site. Even though a minimalistic HUD is shown to be helpful for pilots, the same cannot necessarily be said for surgeons when they have already been heavily trained to understand information in a completely different process. Understanding numbers and lines during flight does not directly correspond to simplistic line drawings of the patient anatomy of the surgical site. Although this study is an important beginning to understanding the effect of two types of overlays during surgery, there are further nuances to be investigated - especially now that a variety of possible rendering methods are available to developers and should be further explored. Transparencies vary from levels of opacity and are not fully fixed as a wireframe model. Further work must be done to fully identify the ideal level of transparency that assists surgeons and enhances available information without obstructing their view.

In addition to work completed by Hallet et. al, work by Dixon et. al. also studied inattentional blindness in laparoscopic surgery - where surgeons complete tasks viewed through an endoscope (a camera in the shape of a long, thin tube, commonly used in MIS procedures). The view from the endoscope is viewed on a nearby computer screen. An AR Overlay was superimposed on top of the surgical view seen in the laparoscope. Users were required to complete a navigation task involving an endoscopic navigation exercise in a cadaver. Two hidden findings were embedded into the cadaver, one resulting in major complications and the other a foreign body (an unexpected screw). The selected participants were all otolaryngology surgeons, trainees, fellows, etc. of different skill levels. Participants were unaware of the two hidden findings upon the start. Users completed the navigation task successfully and were asked afterwards if they noticed anything out of the ordinary that did not pertain to the navigation task they were asked to accomplish (i.e. if they noticed any of the hidden findings). In the control group (no overlay), 
7/17 participants detected a complication and none of the participants who used the AR overlays found the complication. In terms of the foreign body, it was detected by $7 / 17$ of the control group and 1/15 of the AR overlay group.

The results of the experiment display that neither the control or the experimental groups were able to accomplish the task well. In all cases, majority of the participants were unable to detect the findings successfully. It is not mentioned why the control group did not perform very well which can be a concern given there was no difference between normal surgery and this experimental set up. It appears that finding the hidden artifacts may have been difficult for many surgeons regardless of AR overlay or not which is questionable regarding the overall integrity of the experiment. However, objectively, the AR group did much more poorly than the control group. More importantly, the experiment used no variation in transparency. Using only a fully opaque virtual overlay further reinforced this concept that a fully opaque AR overlay may not be useful for surgeons while completing a task and increase inattentional blindness and generally obstruct the view of the patient anatomy [49][50]. In addition to using the AR overlay, it is also not mentioned whether any of the surgeons had any prior experience with AR technologies which is important when studying inattentional blindness.

Interestingly, literature over time consistently mentions that an increase in cognitive load also increases the probability of inattentional blindness. Using an AR system with an audience that may have never had the opportunity to experience AR before causes a large increase in cognitive load and directly affects the level of inattentional blindness [46]. This phenomenon further reinforces the notion put forth in the previous chapter regarding the slow, evolutionary introduction of AR integration within an existing, already deployed system for better user transition. Users should be eased into the idea of a fully AR system that can provide anatomical patient overlays lest increased cognitive load lead to poorer outcomes despite improvements to workflow or accuracy.

Another barrier that AR faces is the physical ergonomics of the devices themselves. The issue of the vergence accommodation conflict refers to the confusion the human brain experiences when wearing these head mounted devices - a problem also faced in virtual reality. Vergence is the length which describes the distance to the object, and the accommodation is the distance where the eyes focus. In the real world, the vergence and accommodation distances are matched. Introducing an AR display shifts this relationship between these distances and causes what is known as vergence-accommodation mismatch [51]. The mismatch influences the quality of the fusion of stereoscopic images that our brains perform. If the mismatch is too great, users can potentially see doubles and experience a degradation in stereoacuity. Stereoacuity refers to the smallest detectable depth difference seen in binocular vision [51]. Multiple works looking to resolve this issue have been done, but a generally acceptable solution has yet to be 
realized [51]. The mismatch is also one of the major causes of eyestrain and headaches for users while experiencing AR and VR environments which is especially apparent after long sessions. For recreational use, users can simply remove the HMD, and endure the consequences afterwards; unfortunately, in surgery, the consequences have larger implications such as patient outcomes.

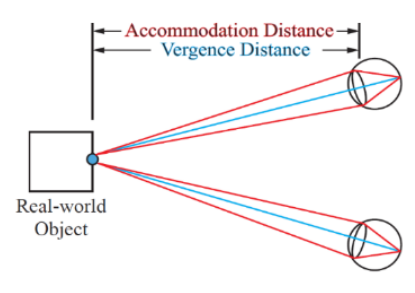

(A)

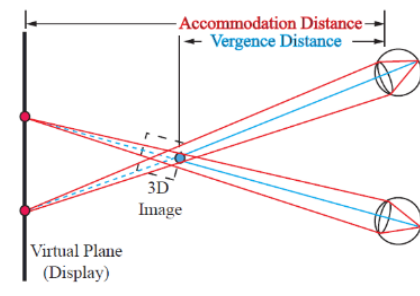

(B)

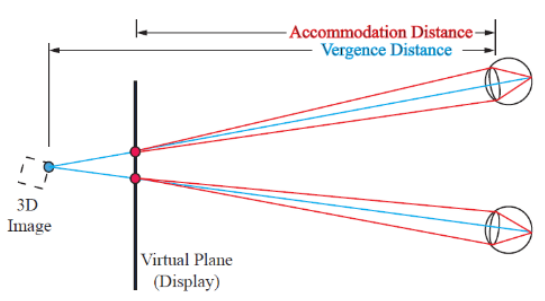

(C)

Figure 20: A) Viewing a real-world object results in matching accommodation and vergence distances. B) A virtual display shows an object close to the observer which results in mismatch as the eyes converge on the display. C) A virtual display showing a far object also results in mismatch. Both forms of mismatch shown in panels B and C result in poor stereoacuity. Figures are adapted from [51].

It is important to note that the AR systems used in existing works are all video see-through devices and some are not using head mounted displays. These studies also take place outside of operating room conditions. Since our work is using a head mounted optical see-through display, the variable lighting conditions of the operating room play a larger role in usability. The bright surgical lights interfere with the way virtual object are presented in an optical see-through display. Virtual objects appear to be less vivid due to the additional light source. An optical see-through display offers some benefits in the operating room, including allowing the user to leverage existing techniques when studying anatomy with minimal modification, and increased comfort in being able to visualize the real-world and the surgical site at all times even when using AR overlays. Using a see-through display also maintains the level of display inertia such that it is less overwhelming for the user to transition from a computer screen to the surgical site. Here, we qualitatively determine the preferred combination of multiple rendering factors, including level of transparency, colour combinations, and number of different layers of the patient anatomy to best visualize hard and soft tissues for medical personnel. 


\subsection{Methodology}

Participants of all skills levels were considered for the study. In total, 9 participants were recruited consisting of 5 surgeons/fellows (1 interventional neuroradiology (INR) Fellow, 4 neurosurgeon attendings/fellows), 2 residents, and 2 medical students. Neurosurgery residency is 6 years long; the year of enrollment for the resident is denoted by "PGY" (which stands for postgraduate year) where PGY-1 indicates the first year of their residency.

Participants were asked to wear the Microsoft HoloLens during non-operative portions of the surgery for up to 10 minutes. It was critical to only ask participants questions during non-operative times of the procedure to reduce the impact on the operating room workflow and maintain both patient safety and the standard-of-care. An application was pre-loaded onto the HMD which displays the patient specific anatomy. The layers displayed were separated by soft (skin, muscle tissue, vasculature, etc.) and hard tissues (bone, tumours, lesions, etc.). The properties - colour, number of layers, and opacity, were changed in real time with the help of a researcher controlling display variations using a Bluetooth keyboard. Since the variable lighting conditions of the operating room are known to affect the optical seethrough display, participants were asked what they thought the desired opacity was so that the external lighting conditions would not skew their preferences. Completely invisible was $0 \%$ and completely opaque was considered $100 \%$. The wire-frame option of rendering the virtual objects was not desirable in this context because of the level of detail seen in CT and MRI images. If the skull is rendered in wire frame, with about 180,000 vertices rendered after down sampling, the entire skull would look opaque at a distance because of the high resolution. To reduce the resolution to the point where the anatomy would appear more see-through would severely degrade the reconstruction quality. At the end of the questionnaire, users would be seeing what their ideal visualization combination was based on their individual preference for an augmented reality overlay system.

Other variables restrained by the physical design of the Microsoft HoloLens including model resolution, ergonomic fit, and lag were rated to further inform future designs of a surgeon-centred HMD. Surgeons were also given the option of wearing the Osterhout Design Group R7s to provide feedback regarding the physical ergonomics of a comparative headset, though no structures were rendered on this headset. Participants were also asked what other structures would assist them in their visualization and other key structures they would be interested in seeing for assistance during surgery. 


\subsection{Results and Discussion}

\begin{tabular}{|c|c|c|c|c|c|c|}
\hline \multirow{2}{*}{$\begin{array}{c}\text { User } \\
\text { Number }\end{array}$} & \multirow{2}{*}{$\begin{array}{c}\text { Experience } \\
\text { Level }\end{array}$} & \multirow{2}{*}{$\begin{array}{l}\text { Displayed } \\
\text { Anatomy } \\
\text { (2 layers) }\end{array}$} & \multicolumn{2}{|c|}{ Transparency } & \multicolumn{2}{|c|}{ Colours } \\
\hline & & & $\begin{array}{c}\text { Hard } \\
\text { Tissue (\%) }\end{array}$ & $\begin{array}{c}\text { Soft } \\
\text { Tissue (\%) }\end{array}$ & $\begin{array}{l}\text { Hard } \\
\text { Tissue }\end{array}$ & $\begin{array}{l}\text { Soft } \\
\text { Tissue }\end{array}$ \\
\hline 1 & Neurosurgeon & Spine and Skin & 100 & 0 & White & $\mathrm{n} / \mathrm{a}$ \\
\hline 2 & Spine Fellow & Spine and Skin & 75 & 30 & White & White \\
\hline 3 & $\begin{array}{c}\text { Interventional } \\
\text { Neuroradiologist } \\
\text { Fellow }\end{array}$ & $\begin{array}{l}\text { Skull, Brain and } \\
\text { Skin }\end{array}$ & 100 & 75 & White & White \\
\hline 4 & $\begin{array}{l}\text { Neurosurgery } \\
\text { Resident }\end{array}$ & Spine and Skin & 100 & 0 & $\begin{array}{c}\text { Yellow or } \\
\text { Orange }\end{array}$ & $\mathrm{n} / \mathrm{a}$ \\
\hline 5 & $\begin{array}{c}\text { Neurosurgery } \\
\text { Resident (PGY4) }\end{array}$ & $\begin{array}{c}\text { Aneurysm and } \\
\text { Surrounding } \\
\text { tissues }\end{array}$ & 100 & 0 & White & $\mathrm{n} / \mathrm{a}$ \\
\hline 6 & $\begin{array}{c}\text { Neurosurgery } \\
\text { Resident (PGY4) }\end{array}$ & $\begin{array}{c}\text { Spine and } \\
\text { Surrounding } \\
\text { Skin/Soft Tissue }\end{array}$ & 100 & 47 & White & Purple \\
\hline 7 & $\begin{array}{c}\text { Orthopedics } \\
\text { Resident (PGY1) }\end{array}$ & Spine and Skin & 100 & 0 & $\begin{array}{c}\text { Yellow or } \\
\text { Orange }\end{array}$ & $n / a$ \\
\hline 8 & Medical Student & $\begin{array}{c}\text { Brain Tumour } \\
\text { and Skull }\end{array}$ & 100 & 40 & Red & Purple \\
\hline 9 & Medical Student & Spine and Skin & 80 & 50 & Green & Red \\
\hline
\end{tabular}

Table 3: Preferred transparency values and colours for each participant.

Regarding feedback regarding the physical HoloLens HMD, users generally felt that it could be comfortably worn for 10 minutes or less at a time. There was little noticeable delay that the users could see with an acceptable resolution compared to a conventional computer screen. Many users have felt some fatigue wearing the HMD for long periods of time because of the way that the weight is distributed on the glasses. Much of the weight is on the placed outwards on the user's forehead and nose. Considering the whole HMD weighs 576g, it can be quite uncomfortable. This awkward placement of majority of the weight also causes users to progressively shift their gaze downwards over time.

Visual fatigue was generally considered acceptable for the small amount of time that it was worn during the surgery. However, visual fatigue and eyestrain in those who already require glasses should theoretically be greater than those who do not require glasses. Since some prescription glasses did not fit underneath the HoloLens, poor vision in combination with the existing discomfort from the vergence accommodation conflict can create greater discomfort.

Other structures that surgeons considered important to view through AR would be key vital structures that must be avoided, such as the vertebral artery going through the foramen transversarium during spine surgery. This artery is very important as it connects to other major vasculature in the body and should 
always remain intact during surgery. Novice surgeons can sometimes accidently shear this vessel and cause further injury to the patient.

\subsubsection{Practical Interpretation and Limitations of the Study}

The results show that the average desired transparency for hard tissue is $95 \%$ and for soft tissue is $27 \%$. Interestingly, the levels of transparency show similar trends across all participants; however, participants which have greater experience (i.e PGY3 resident or above) prefer levels of transparency closer to $0 \%$ for the soft tissue except for the INR fellow with soft tissue transparency at $75 \%$. A possible reason for this exception could be because interventional neuroradiologists observe similar transparency between soft and hard tissue comparatively when using fluoroscopy. Analogously, other surgeons which often depend on bony landmarks seen in CT imaging prefer little or no soft tissue to be visible while the hard tissue would be highly or almost entirely visible.

It is also interesting to note that white is most useful for participants with experience equal or greater than PGY-3 prefer only white for their renderings. For those who are below PGY3, they tend to prefer using contrasting colours that make unrealistic renderings to denote soft and hard tissue. A possible explanation for this could be that by PGY-3, residents have become accustomed to studying computed tomography (CT) and Magnetic Resonance (MR) images which are normally grey-scaled with intensities representing various densities, structures, etc. Similarly, junior trainees who may be less accustomed to looking at these images may be more open to varying colours which give more contrast to tissue layers.

These qualitative results further inform future initiatives in design choices for developing a surgeoncentred augmented reality system for the operating room. Although more usability tests are required to fully understand the surgeon's perspective on an AR-HMD, this test further informs users of the difficulties involved with using an optical see-through HMD for use in an operating room with variable lighting conditions. This work also confirms that information should be displayed like other imaging modalities (i.e. CT or fluoroscopy) for effectively displaying information to surgeons.

With regards to the previous chapter, where a slow integration of AR is important, an HMD for surgery may sound like a large jump from a simple line overlayed on a computer screen. The experience of using a computer screen is very different and much less immersive compared to an AR HMD. However, glasses offering multiple views - one normal, and one at a fixed magnification, are already common-place in the OR, and are known as surgical loupes. Surgeons interchange the two views (normal and magnified) by peering up or down through the surgical loupes. The availability of information displayed through a pair of glasses changing through shifting their gaze is very common in the operating room already. In this, 
creating an iterative design that acts as "augmented" loupes can allow for a sort of "evolutionary change". Although many technology reviewers of the Microsoft HoloLens may say that the limited field of view of the HoloLens is a disappointment because the viewing window is so small, it can potentially be beneficial during surgery. Surgeons would have the ability to experience a normal surgical site as well as an AR surgical site through changing their gaze. Shifting the information being displayed without requiring the surgeon's hands is also advantageous to maintain a sterile surgical site.

An important factor to address regarding AR HMDs is the potential introduction of lag. Numerous systems in the operating room such as a microscope, ultrasound systems, etc. have very little latencyespecially optical dependent systems. Introducing latency decreases the accuracy of specific tasks and increases frustration in users [52]. From our own experiences, we have seen users adapt to the change in lag by attempting to complete the task at a slower rate. Although it is possible for users to adjust to a speed according to the lag, it is inconvenient and highly undesirable.

Weight distribution is the ultimate drawback of a system like the Microsoft HoloLens. There is an unfortunate trade-off between many HMD designs where they must choose between comfort or computing power in their final designs. Majority of the weight of the HMD is situated at the front of the user's face on top of the nose bridge. Considering the glasses weigh $576 \mathrm{~g}$, one can understand why wearing the headset for a long time can be difficult - especially when considering the length of many surgeries is often several hours. Given that the posture of a surgeon is more upright while operating, it may be more beneficial to place the weight towards the back of the head closer to the base of the skull or neck area. Other systems are tethered to a computer to offload these components leaving the only the lenses on the user's head for better long-term wear. Although more comfortable, users have a smaller range of motion as they must remain connected to their computers. An increasingly popular method of decreasing the overall weight of HMDs is to provide a secondary attachment which fits into a pocket. This attachment usually contains computing components or batteries. Not only does using this secondary attachment decrease general user discomfort and overall weight while improving battery life and computing power, but also maintains the mobility and functionality of the device.

This study could see improvements through studying the impact of the selected rendering properties that the participants decided was optimal for visualization. Although surgeons can choose in real time how the renderings are displayed, it does not necessarily mean that under operative conditions the information displayed would be effective to accomplish a specific task. With no task at hand for the use case, there also is no quantification for inattentional blindness and overall helpfulness as seen in previous studies. 
However, considering this test is based on an inaccurate registration (as the focus of this test was to determine optimal visualization settings), the experimental design does not provide an accurate framework to measure inattentional blindness. If surgeons were asked to complete testing focused on inattentional blindness using this experimental design, the results would be skewed due to the inherent error in the registration accuracy of the Microsoft HoloLens, as is discussed in the next chapter. Previous studies which did recreate a perfect registration were not using optical see-through head mounted displays and did not provide a true augmented reality experience. These works were all using an AR environment established by a video see-through set up (either using a video see-through HMD or a camera feed with the patient anatomy superimposed onto the video feed displayed on a computer screen).

A further limit to the study can be seen in the total number of participants. While a varied cross-section of a neurosurgical program was surveyed, the study was limited by access to participants, and only sampled from a single institution. Further, with only a handful of participants from each group being surveyed, rigorous conclusions cannot be drawn, and further studies from multiple participants in each level of training, and from multiple institutions where practices may vary, should be carried out for firm conclusions to be drawn. However, the above does represent a sufficient cross-section for a single centre, and upon which some direction for future design can be gleaned.

Overall, an understanding the ways in which surgeons visualize information best is imperative in the design of an HMD built for the operating room. Determining common trends and running various usability tests is important for reflection on future AR designs which improve cognitive performance under stressful situations. Considerations such as inattentional blindness and cognitive overload are very real concerns in a medical setting, but can be navigated and avoided through careful and deliberate design choices. The difference in rendering virtual content in varying transparencies may sound trivial, but has been shown to produce very different outcomes. By showing that surgeons express a high level of cognitive directness, UI (User Interface) designers have the capability to display information that is incredibly intuitive for the main user audience. Rendering the AR overlay which appears like commonly used medical imaging modalities poses a method for showing information without the need for explanation or learning what they are viewing through the HMD. Additional factors such as ergonomics and physical design are vital for a well-integrated operating room system. Without ensuring long term wear, practicality, weight distribution, and other user-centred design choices, the technology loses likelihood for adoption into the operating room workflow. While a better understanding of ergonomic form factor and how surgeons may best visualize augmented reality overlays has been gained, the inherent accuracy of the system must be quantified - as is discussed in the next chapter. 


\section{AUGMENTED REALITY PLACEMENT ACCURACY}

\subsection{Preface}

Although the human computer interaction portion of device development is incredibly important for adoption, the utmost importance for medical devices is to be accurate. If a system is not accurate enough, it should not even be considered for use in an operating room. As such, various systems have an extensive testing program to ensure that they can be certified for clinical use and have demonstrated a capability to be reliable and repeatable. Regarding AR surgical navigation systems, this testing process is not concretely defined considering there is no existing stand-alone AR device for navigation. All existing AR based navigation systems are dependent on the same technology as previous iterations with the IR tool tracking system. The purpose of this specific study is to determine how precisely existing commercially available AR HMD systems can place virtual patient anatomy to the real physical patient anatomy.

Currently, several research groups have investigated placement accuracy of the Microsoft HoloLens in the interest of furthering the agenda for fully AR guided neurosurgery. Each of these measurement processes have yielded different results and are not convergent despite being from the same system. The Microsoft HoloLens has been commonly considered for research in surgical AR because of its inherent capability to map the physical world around it. Simultaneous localization and mapping (SLAM) capability was unique to the HoloLens at first as it was one of the only HMDs at the time of release to have this ability. SLAM is important for AR HMDs because it greatly improves the AR experience for the user. Without SLAM, AR systems would not be able to place virtual content into the real-world space if at all. If SLAM is implemented poorly on an AR system, the virtual content tends to "drift" or "jitter". These phenomena are highly undesirable for an AR system as it creates an unrealistic illusion of a true AR environment. It is important to understand SLAM and its significance to AR to further understand the purpose of determining the placement accuracy of an AR system.

SLAM is a problem which originates from an issue in the field of autonomous robotics. The purpose of autonomous robots is to further exploration without the use of physical humans. At times, spaces to explore may be too dangerous for humans or too narrow for an adult to physically enter. The goal of SLAM is the ability to create a map of an unknown environment. The issue is analogous to the phrase "what came first, the chicken or the egg?". In terms of robotic mapping, the question is "How do I know where I am if I do not have a map? But how do I create a map if I do not know where I am?” [53].

SLAM has been attempted to be solved in different methods. One of the first methods is through "dead reckoning" which is purely basing location and mapping on sensor data. An example would be 
implementing an autonomous robot which uses wheels to explore the unknown space. Hypothetically, the circumference of the wheels, the number of turns that wheels have turned, and the direction which the robot is moving (based on accelerometer data) is all known information. Based on this known a priori, we can infer the new location of the robot. However, this method of SLAM is unreliable over time because of sensor drift [53]. Dead reckoning is not an effective method of SLAM for autonomous robotics but was an important start for this field.

Later, the Kalman filter was implemented developed from control theory and heavily influenced the field of autonomous robotics. The Kalman filter was initially introduced as an optimal recursive solution to filter discrete linear data. It has been shown to be able to predict past, present, and even future states based on existing known measurements. The Kalman filter is considered "optimal" because all knowledge of the system is taken into consideration, regardless of precision of previous values, to predict the next value [54]. The model consists of two parts: the prediction and the update phase [55][56]. The Kalman filter is based on state space models where the "states" are dependent on the parameters being observed. It is important to note that the Kalman filter is biased towards linear models. To adapt the Kalman filter for SLAM, an Extended Kalman Filter is used (EKF). The EKF is an extension of the Kalman filter but is used for non-linear data (within reason as the foundation is still built upon a linear model) [55]. The prediction and update phases of the EKF are replaced with two new states: localization and mapping phases of SLAM. Because of the recursive pattern of the Kalman filter, the localization and mapping can happen simultaneously as it continues to add to the existing data as it creates the next prediction.

Since the EKF is still seen as an extension of the Kalman filter, there is still some inherent bias to linear systems in comparison to the particle filter. To use the EKF, the non-linear and non-Gaussian incoming data must be linearized through a first-order Taylor Series Expansion. The EKF has the potential to be very computationally expensive, especially for higher dimensional data (i.e. the heights of walls would be 3D SLAM. The floor topology of an unknown space would be 2D SLAM) [57]. The particle filter is a more flexible system for SLAM as it is more heavily based on statistics and Bayesian probability [57]. The particle filter is quite appealing for developers because of its robustness towards non-linear incoming data (such as an unknown and unexplored environment). However, as the mapping capability increases in dimension (from 2D SLAM to 3D SLAM), the number of points required increases.

In terms of AR, SLAM is becoming incredibly more important in the field for enabling a further convincing AR experience for the user [58]. SLAM allows for the AR system to understand the surrounding world in its own way, enabling it to superimpose virtual content that can interact with the real world directly. Without SLAM for the HoloLens, virtual objects would not be able to be placed on top of a desk or a table. Although it would sound simple for a human to place an object onto a table, the same 
kind of understanding and comprehension of what a table top is and how to manipulate an object is a struggle for machines to understand. When using the HoloLens, users can directly see what the HoloLens understands as a surface in a very vague representation of flat surfaces. The maximum density of spatial mapping of the HoloLens is 1400 triangles per cubic metre [59]. Each of the triangles vary in sizes while creating a spatial map, but one can visibly see that the sides of the triangles are in the "cm" range which is suitable and realistic for mapping an entire room and given the limited computing resources of the headset.

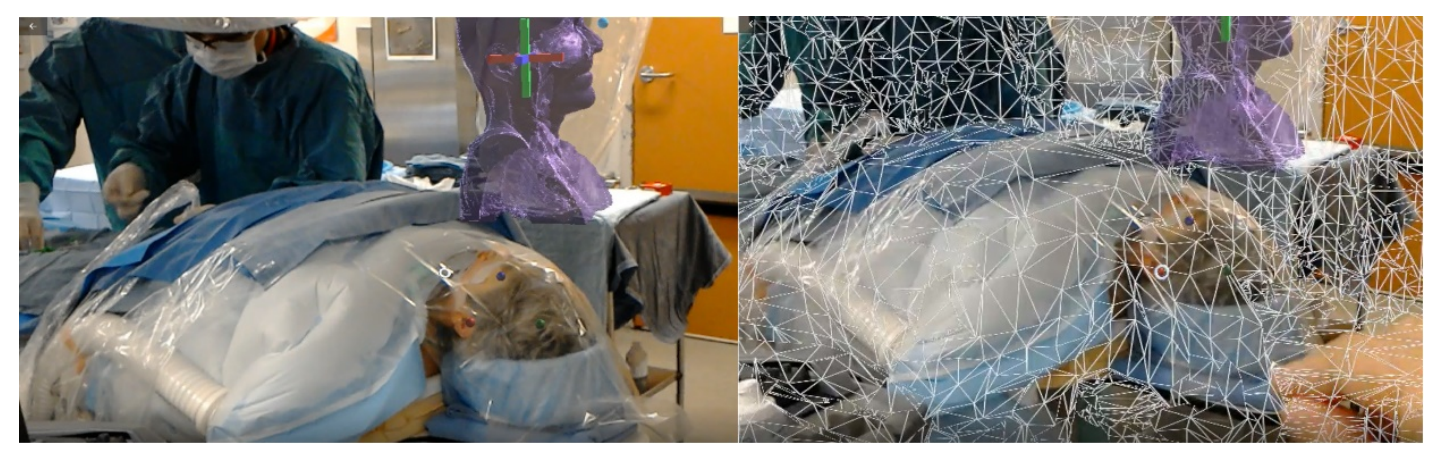

Figure 21: An example of the spatial mapping capabilities in the angiography suite during an interventional neuroradiology case. The patient anatomy shown in purple is in the process of being placed onto the physical patient. The left panel is the normal mixed reality view while the right panel is the spatial mapping capability of the HoloLens used to better understand the surfaces in the room.

Recognizing this need for SLAM in AR, Microsoft developed a Mixed Reality Toolkit which was simple for developers to implement. This mapping capability makes it quite appealing for surgical AR research. One of the overarching motivations for surgical navigation is to make "pseudo x-ray vision" where surgeons can see through the surface of the patient while operating. Ideally, the resulting view would be helpful, especially for MIS neurosurgery, to potentially shorten operating times and lessen associated costs while maintaining a high level of patient care. In MIS procedures, the surgical site is significantly smaller as compared to with open surgery, creating less landmarks for surgeons to visualize and work from. The result is that traditional OR training where surgeons localize themselves based on landmarks is no longer applicable and having a system which allows for landmark visualization such as in an AR system would help in overcoming this hinderance.

Several groups have tested various placement methods and placement characterization techniques. Groups have mostly tested one method of placement in their works. Most groups have characterized the placement accuracy of the HoloLens through the "tap to place" method. Tap to place is a script found in 
the mixed reality toolbox made by Microsoft. There are two main input methods required from the user which are "Gaze" and "air tap" [60][61]. Gaze refers to what is known as the ray cast. This input refers to the direction which the user is facing. The HoloLens uses Gaze input to orient the cursor of the system. The cursor is analogous to the common computer cursor except for the $3 \mathrm{D}$ interaction. Interestingly, this cursor also serves as a joint between the virtual and real aspects of the augmented reality experience. The HoloLens cursor can rest on surfaces such as walls and tables and even other virtual objects. The ability to rest the cursor on a physical object will depend on the HoloLens' SLAM ability and if it is able to locate the target in the virtual map of the space [62].

The other required input is the air tap which is a distinct hand gesture which resembles clicking a mouse [61]. Using gaze to place the cursor on the 3D virtual object followed by an air tap, the virtual object will then follow the direction of the gaze until the user does a second air tap to release the object from the ray cast. Rotation is handled through physically walking from one point of view to another. The object stays in the same default orientation and location regardless of how the user's head moves and regardless of the location in the room because of the SLAM capability. The headset is constantly aware of its position within the room because of the salient features it finds.

Vassallo et al. also use the Microsoft HoloLens to address the placement error caused by drift when using the SLAM capabilities and the tap to place function [62]. The disruptions to SLAM addressed here are walking, sudden head acceleration, occlusion and insertion. The displacement was measured using a tracked IR camera system which is commonly found in many navigation systems. Using an IR tracked stylus, the user placed the tool tip to salient features in the hologram seen through the HoloLens. After each of the disruptions were completed, the user then re-registered the tool tip to the new location of the hologram. The mean displacement error in all directions was found to be $5.83 \pm 0.51 \mathrm{~mm}$ [62].

Rae et al. also used the HoloLens to measure the placement accuracy using hand gestures [63]. The work proposed was determining the placement accuracy of the "tap to place" function from the HoloToolKit repository made by Microsoft. In the proposed system, the virtual object was generated from a CT scan of a phantom head to simulate data from a real patient. The CT scan was used to develop a virtual model of the phantom head and was then used for virtual placement on the HoloLens. Measurements were taken in a single direction where the user was standing after the tap to place was completed. The difference in placement was repeated by users of different skill levels and measured in the same area depicted by three markers on the physical phantom head. The results of this study showed to be accurate enough for surgical procedures such as neurosurgical burr hole placement [63]. Although the results appear to be promising for current state of the art systems, the authors also mention that a full 3D measurement system 
to determine displacement and rotational errors in all dimensions should be developed for a better representation of placement accuracy.

Similarly, another work by Fatih et al. also uses a qualitative method for determining placement accuracy. This group places a patient specific overlay directly on top of the surgical site of real patients showing the tumour as well as the skin. Using the outlined boundaries of the tumour as a reference to infer registration accuracy, surgeons would describe the placement to be acceptable or unacceptable from their point of view through the HoloLens [64]. The alignment was compared to standard navigation, where the tracked tool was used to outline the boundaries of the tumour seen through the HoloLens. By tracking the path of the tracked tool outlining the AR tumour, this information can be compared to the true boundaries of the tumour seen on the CT scans on the navigation sytem. This process was completed for 25 patients and it was found that 9 of 25 cases displayed no difference between the CT and the AR overlay. Considering the process is completed from a single view, which is that of which the surgeon can see, this method of comparison to $\mathrm{CT}$ is also seen as a $2 \mathrm{D}$ characterization [64].

Gibby et al. later proposed another system for placement accuracy quantification using the NOVARAD system which is commercially available and fundamentally runs on the Microsoft HoloLens [65]. This system is proposing a visualization aid for surgeons in terms of presurgical planning. However, this group uses the NOVARAD to insert needles into pedicles of the vertebrae. The NOVARAD system uses an algorithm to best fit the spatial map of the room to the $3 \mathrm{D}$ virtual patient anatomy as best as possible, but it does not always seem to be a perfect fit. Users are also given the option to manually adjust the position of the virtual object using an interface until the user is pleased with the placement. Considering that the users have many liberties in deciding if the placement meets their needs, it is evident that the placement accuracy is dependent on what the user believes is accurate enough. The users were told to view the phantom in different views to obtain a more accurate representation of the surface prior to the placement. Their measurement method includes planning on the virtual phantom where the intended trajectories are to go and compared them to the resulting trajectories after the insertion through a secondary CT scan [65]. The results of this placement study also appeared to be suitable for needle insertion into the spine pedicle. Although each these methods were developed to measure the placement accuracy of a virtual object, they do not measure the accuracy between different methods of placement available. All the placement methods investigated in previous studies are also heavily dependent on the spatial mapping capability of the HoloLens to varying extent. The method proposed in this section builds on top of the progress of these contributions to further quantify the placement accuracy of the AR system through measuring displacement and rotational error in all directions. There are three main placement methods to be tested which are "tap to place", 3-point correspondence, and finally manually using a keyboard. Although there 
are other methods such as using an Xbox controller, marker tracking, and hand tracking, they were not included for this study.

The Xbox controller was very similar to the keyboard controller as the number of key strokes is directly correlated to how the virtual object will move. Some commands of the xbox controller have variable sensitivity or acceleration, but these can also similarly be programmed into the keyboard commands.

Marker tracking is a well-known method for interacting with augmented reality and is among one of the most popular. A marker is a printed pattern which contains many easily distinguishable features. These features are characterized in a way such that the marker is easily trackable. After the marker is defined, it can be identified in a scene given that it is visible to the tracking camera. When the location and orientation of the marker is determined, a virtual object can reflect the same location and orientation of the marker. Users have a physical object to manipulate, like the real world, making it very intuitive.

Some groups such as Tristan et. al. and Florentin et. al. use marker tracking in order to track a tool within the initial HoloLens coordinate system. Tristan et. al. uses the marker to track a stylus tool in order to describe 7 points on the physical patient to the HoloLens. A best fit was performed between the 7 points and the CT scan. Their evaluation methods were based on both target registration error (registration error attributed to the process) and the fiducial registration error (registration error due to the marker tracking). The combination of these two errors was found to be $7.2 \pm 1.8 \mathrm{~mm}$ which is relatively high for a neurosurgical setting [66]. Florentin et. al. also uses the marker to track a stylus. However, this group uses the stylus to outline the general visible region of a spine pedicle (which is the coronal view of the spine). They outline or shade the entire surface of the visible pedicle. The indicated surface is then best matched to the surface of the specified pedicle. This alternate description of a surface is like that which is done in brain surgery for some cranial navigation systems such as the one offered by Medtronic. Error was categorized through the comparison between planned and executed angles for pedicle screw insertion, which is considered acceptable by the surgeons involved in the study. The primary mean error from this method was found to be $3.38^{\circ} \pm 1.73^{\circ}$. The AR placement accuracy here is not only dependent on the surgeon's discretion, but also only regarding the rotational error with respect to the screw entry point [67]. Considering that the HoloLens is inherently some kind of PTAM (Parallel Tracking And Mapping) tracking system, it is also known that this system is inherently favourable towards rotational error compared to translational error [68].

Frantz et. al. is another group that use fiducial markers, but for registration purposes. The target skull was placed on a grid such that the distance between the marker and the skull was explicitly defined and easy to implement in the Vuforia marker tracking Software Development Kit (SDK). Since marker tracking is 
only measurable in one direction (enface to the marker), measurements were taken at angles with respect to the perpendicular axis to the marker (which would be the optimal view for the marker at $0^{\circ}$ ). Angles were measured from $-90^{\circ},-45^{\circ}, 0^{\circ}, 45^{\circ}$, and $90^{\circ}$. Overall error was found to be $1.29 \mathrm{~mm}$ [69]. Although given a high accuracy, marker tracking is still only a 2D solution given that the marker can only be viewed from a single direction. Theoretically, three markers would then provide a 3D solution, but may not necessarily be helpful in a neurosurgical setting where it could potentially be difficult to implement.

Although this method can be reliable for most AR applications, it is unsuitable for surgery for multiple reasons. When observing the virtual object with respect to the marker, the marker must always be in view of the camera. Measuring the displacement and the rotational error in all axes is not possible as two of the three axes involve the marker to be out of view. This can be remedied by implementing three markers in each direction perpendicular to one another, which is impractical and unrealistic in surgery. Surgeons would be required to ensure that the markers are always in view of the camera to render the virtual patient anatomy. It is also difficult to ensure that the marker is always flat; especially in brain surgery where the human head is curved. Placing three perpendicular markers onto the surgical site can also potentially be too time consuming and disruptive to the OR workflow.

Hand tracking is an input method which is also a Unity C\# script found in the Mixed Reality Toolkit. After air tapping (as described in the tap to place method), the user maintains the "tapped" position and the virtual object will move with the user's hand motions. Releasing the tapped position will allow the user to put the object down in the surrounding world space. Hand tracking is analogous to "drag and drop" motions on a normal computer. Although this input method is intuitive for most users, it is very clumsy; especially for refined placement.

Another interesting medical AR group by Molina et. al. proposed the development of a new system which does not use the Microsoft HoloLens but uses their own custom hardware and software to create a new device. Molina et. al. use a system from Augmedics Ltd. For this study, researchers use a cadaveric spine with an embedded metal marker which can be detected on the CT scan. The alignment was completed using the visible marker in the $\mathrm{CT}$ as the virtual reference and aligning it to the physical reference marker on the surgical site. Regarding their registration accuracy, there was no concrete measurement other than the considerations of the surgeons engaging in the study, deeming it to be acceptable or unacceptable. Although it is a method of AR registration which has not been completed before, this process of embedding a marker into the patient pre-surgery is incredibly invasive and increases the potential for infection. Not only is the surgical site exposed for a longer amount of time, but the implantation of the metal marker itself is almost a low-level surgery on its own prior to the correctional surgery. Especially in 
life-threatening, urgent cases, it may not be feasible to perform the implantation prior to the actual correctional surgery and indubitably does not work with the operating room workflow for these cases.

\subsection{Methodology}

As previously mentioned, the three placement methods are:

Tap to Place

3-Point Correspondence, and

Keyboard Placement.

\section{1) Tap to Place}

Tap to place is a script found in the mixed reality toolbox made by Microsoft. This placement method was described in the Preface of this chapter, and as previously mentioned this placement method is highly dependent on the SLAM capability of the HoloLens. The HMD maps the room, identifying where large surfaces such as walls, tables, and the floor are. Required input is the air tap as done through a hand gesture. Using gaze to place the cursor on the 3D virtual object followed by an air tap, the virtual object will then follow the direction of the gaze until the user does a second air tap to release the object from the ray cast. When the object is released, the virtual object is left resting on a mapped surface. Rotation is handled through physically walking from one point of view to another. The object stays in the same default orientation regardless of how the user's head moves.

\section{2) 3-Point Correspondence}

3-point correspondence is a matching algorithm based on best fit between two sets of three points. 3 dots are readily placed onto salient features of the virtual object when it is rendered on the HoloLens. The user is directed to place another set of colour-correspondent dots onto the real object as closely as possible to mimic the placement on the virtual object. The moveable set of points which are placed on the real-world object are moved using the tap to place method mentioned prior. After both sets of dots are in the correct place, the user presses a key on the Bluetooth enabled keyboard to calculate the rotation and translation between the two sets to best match them. 
To accomplish this, we must think of 3 dots on the virtual object as a set and the moveable dots to be placed on the real object as a separate set. We will call the dots $V P_{1}, V P_{2}, V P_{3}$ (Virtual Points 1 to 3 ), and $R P_{1}, R P_{2}, R P_{3}$ (Real Points 1 to 3 ). The centroid $C$ is found in each of these sets of points creating $C_{\text {real }}$ and $C_{\text {virtual }}$.

$C_{\text {real }}=\left(C_{\text {real }, x}, C_{\text {real }, y}, C_{\text {real }, z}\right)=\left(\frac{R P_{1, x}+R P_{2, x}+R P_{3, x}}{3}, \frac{R P_{1, y}+R P_{2, y}+R P_{3, y}}{3}, \frac{R P_{1, z}+R P_{2, z}+R P_{3, z}}{3}\right)$

and

$C_{\text {virtual }}=\left(C_{\text {virtual }, x}, C_{\text {virtual }, y}, C_{\text {virtual }, z}\right)=\left(\frac{V P_{1, x}+V P_{2, x}+V P_{3, x}}{3}, \frac{V P_{1, y}+V P_{2, y}+V P_{3, y}}{3}, \frac{V P_{1, z}+V P_{2, z}+V P_{3, z}}{3}\right)$

To determine the translation, each of the points are made to be children with respect to the centroid location. The location of $\mathrm{C}_{-}$virtualis then moved to match the centroid $\mathrm{C}_{-}$real found by determining the difference in location $\mathrm{T}$ between the two centroids.

$T=\left(T_{x}, T_{y}, T_{z}\right)=\left(C_{\text {real }, x}-C_{\text {virtual }, x}, C_{\text {real }, y}-C_{\text {virtual }, y}, C_{\text {real }, z}-C_{\text {virtual }, z}\right)$

After the centroid locations are finalized by the user, the spatial mapping capabilities of the HoloLens are then disabled from this point on. If the spatial mapping were to remain enabled, it would create a virtual barrier for the virtual object to cross, preventing it from aligning as close as possible.

Next is to describe the Rotation to match the virtual set to the real set. Each set of points can also be described in relation to each other by creating a plane. As we know from linear algebra, a plane is described by two vectors which is established from three points.

$$
\begin{aligned}
& \overrightarrow{R_{1}}=R P_{1}-R P_{2}=\left(R P_{1, x}-R P_{2, x}, R P_{1, y}-R P_{2, y}, R P_{1, z}-R P_{2, z}\right) \\
& \overrightarrow{R_{2}}=R P_{1}-R P_{3}=\left(R P_{1, x}-R P_{3, x}, R P_{1, y}-R P_{3, y}, R P_{1, z}-R P_{3, z}\right)
\end{aligned}
$$

and

$$
\begin{aligned}
& \overrightarrow{V_{1}}=V P_{1}-V P_{2}=\left(V P_{1, x}-V P_{2, x}, V P_{1, y}-V P_{2, y}, V P_{1, z}-V P_{2, z}\right) \\
& \overrightarrow{V_{2}}=V P_{1}-V P_{3}=\left(V P_{1, x}-V P_{3, x}, V P_{1, y}-V P_{3, y}, V P_{1, z}-V P_{3, z}\right)
\end{aligned}
$$


The vectors $\underset{R_{1}}{\rightarrow} \underset{R_{2}}{\prime} \underset{V_{1}}{\rightarrow}$, and $\underset{V_{2}}{\rightarrow}$ are then used to determine two normal vectors $N_{\text {real }}$ and $N_{\text {virtual }}$ through a cross product which are then used to create two planes $P_{\text {real }}$ and $P_{\text {virtual }}$.

The angle between the $N_{\text {real }}$ and $N_{\text {virtual }}$ is found through a dot product. $N_{\text {virtual }}$ is then rotated by this angle to match the direction of $N_{\text {real }} . P_{\text {real }}$ and $P_{\text {virtual }}$ now lie on the same plane. Rotation about the normal is then performed to determine the best match of VP1 to RP1, VP2 to RP2, and VP3 to RP3 respectively. The rotation is determined by the best fit between the two planes.

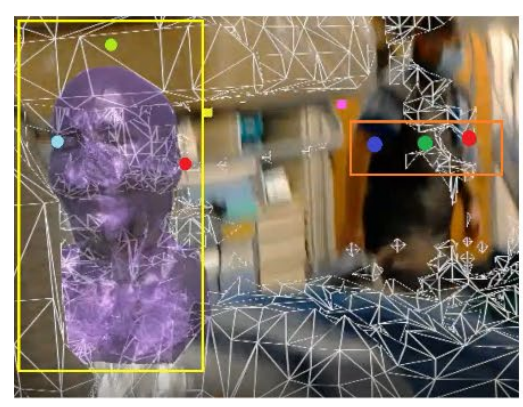

(A)

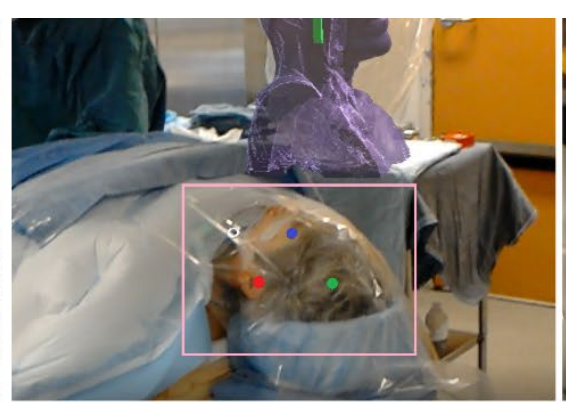

(B)

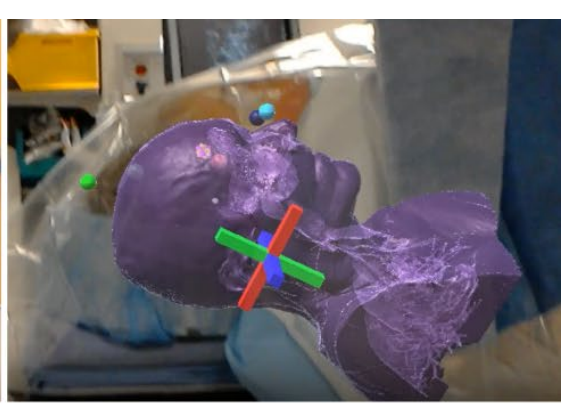

(C)

Figure 22: Registration process for 3-point correspondence. A) upon start up, the application activates spatial mapping at the highest possible resolution. The yellow box displays the virtual patient anatomy with virtual points pre-decided. The orange box shows the three real world points. B) The user places the real points onto the physical patient corresponding to the pre-decided points shown in the pink box C) Best fit is determined based on the centroids and normals of the virtual and real coordinates.

\section{3) Keyboard Placement}

This method is not dependent on the spatial mapping capabilities of the HoloLens other than the location of the origin upon startup. The placement of the virtual object was completed manually using a Bluetooth keyboard which connected to the Microsoft HoloLens. The controls were set in such a way where W, A, S, D keys were used to move forwards, backwards, left, and right (to mimic computer game keyboard control), and the rotation was controlled using J, K, L keys. These controlled rotations about the X, Y, and $\mathrm{Z}$ axes respectively. The XYZ axes are initialized upon start of the application. The WASD keys move the virtual object in increments of $0.5 \mathrm{~cm}$. Users were asked to align the virtual phantom as directly onto the real phantom as closely as possible using only the keyboard to manipulate the object.

Each of these methods were completed by three people of varying experience with AR technologies; one person used it on daily to weekly basis, another on a monthly to bi-monthly basis and finally, one person 
who has never used it before aside from the basic introductory user tutorial. Each person of a different skill type completed each method three times per phantom to ensure interrater error was taken into consideration. Multiple operators with varying skill level participating in the study was necessary for obtaining a realistic comparison of the different end users in the operating room. It would be unrealistic to only have one person complete every trial in in every surgery.

Four different phantoms were used in this study; a head, spine, percutaneous phantom, and a calibration block. A variety of targets for placement accuracy were used to give an idea of different possible applications which can be done in the operating room. Phantoms of different sizes also allows each method to be given an equal evaluation. Each phantom also contains varying levels of detail which consequently, require different levels of sensitivity for placement. Different methods of placement mentioned here may also have a bias depending on the real-world target and which functions are used to develop the application. Due to the spatial mapping capabilities of the HoloLens, there is an affinity for mapping objects which have large flat surfaces (i.e. walls, floors, and tables).

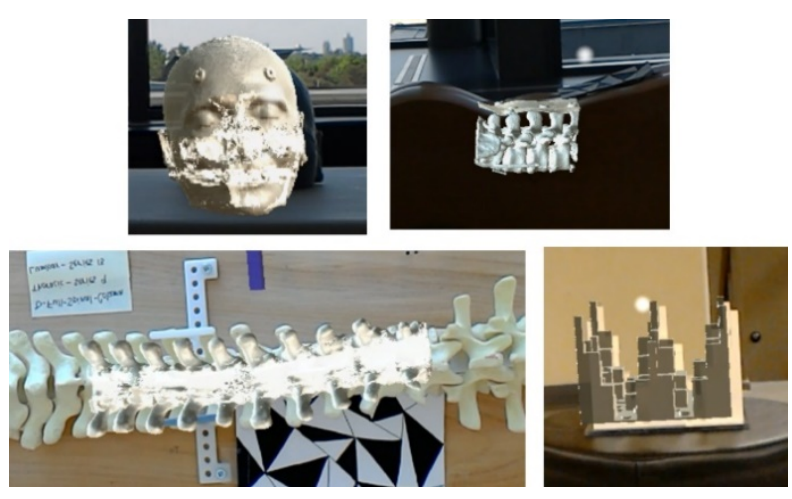

Figure 23: Various phantoms used for placement testing of all methods. First row from left to right are the head phantom and a percutaneous phantom. These two phantoms are the relatively smaller level of detail. The bottom row, a phantom spine and a calibration block have a greater level of detail.

In addition to the phantom data, the experiment was repeated in a clinical setting to determine if the same accuracy could be reflected in a real-world environment. Placement methods were tested in the operating room during non-operative times such as during anesthesiology prior to the first cut during surgery. After the patient is draped, there are no salient features visible for the measurement process. Incorporating elements in a real functional operating room also ties into earlier chapters discussing the operating room workflow and what factors can be attributed to the change in accuracy between the placement methods in the model and the operating room. 


\subsubsection{The Measurement Process}

Each phantom was placed in a CT scanner from which virtual 3D reconstructions of each phantom were created. From here, the models were used for each of the placement methods. The main objective was to use each of these methods to align the virtual object with the real object as closely as possible. Each placement method was repeated three times per user and per phantom to the best of their ability. During each trial, users were recording views perpendicular to the phantoms to obtain a clear view in XY, YZ and XZ planes. A clear view of each plane then yields various displacement and rotational errors in registration during post processing. Each view provided three displacement errors. These measurements were taken at the most extreme, minimum and average rotational and displacement errors to ensure an average value. Initially, all measurements were quantified in pixels from the recordings. Each view also contained a $100 \mathrm{~mm}$ scale bar which was aligned on the same plane as the virtual models. After measuring the number of pixels in the scale bar, a scaling ratio was created for each view of each trial. The errors measured in pixels were converted to mm. For rotational error, two vectors which were known to lay on the same plane were chosen. One vector was outlined on the virtual phantom model and the other on the real model. A dot product was done between these two vectors to determine the angle between them. The dot product yields the angle between the two vectors. Similarly, to calculate the displacement error, a difference between salient virtual and phantom features were measured.

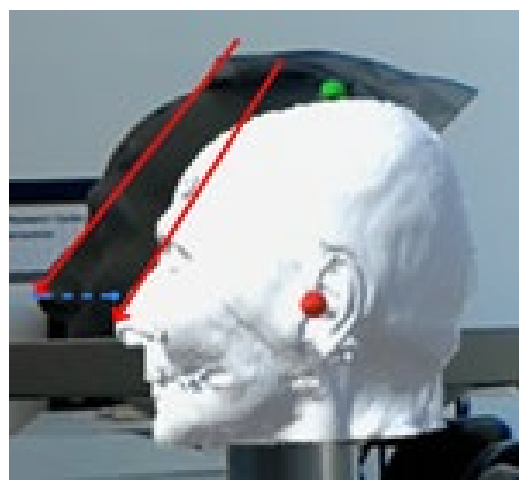

Fig. 24. Virtual patient anatomy is overlaid in white and the real patient anatomy is shown in dark grey. The vectors outlined in red show the rotational error between the real and virtual models (determined by the dot product). The blue line shows the displacement error between salient features (in this case, the nose tips).

A total of 27 trials were completed with 2049 measurements taken in total for displacement and rotation. All measurements were completed by one person which also took a click accuracy test. This test determined the amount of error which was inherent during the measurement process. For this test, multiple circles were generated at random sizes and locations on the same computer screen which would 
further be used to take measurements. The user was required to click the centre of the circles as best as possible. The straight-line distance from the real centre of the circles and the clicked centre was determined each time. The average error for the user was taken into consideration when the images were post processed. The results of the click accuracy of the designate evaluator is reflected in the results section found below.

Following the phantom experiments, a power analysis was completed to determine the number of patients was required for statistical significance. The power analysis is based on five main factors: power, significance level, effect size, number of groups, and sample size. Power and significance level are generally kept at 0.8 and 0.05 respectively- most power analyses will not change these values. There are three groups (which are the three placement methods), effect size was found from the ANOVA completed earlier, and the power is what will be determined based on the other four parameters. As indicated from the power analysis, 18 patients were required for statistical significance.

\subsection{Results and Discussion}

\begin{tabular}{|c|c|c|c|}
\hline & XY Plane (mm) & YZ Plane (mm) & XZ Plane (mm) \\
\hline Tap to Place & $3 \pm 2$ & $3 \pm 2$ & $4 \pm 3$ \\
\hline $\begin{array}{c}\text { 3-Point } \\
\text { Correspondence }\end{array}$ & $7 \pm 4$ & $5 \pm 4$ & $6 \pm 4$ \\
\hline Keyboard Placement & $3 \pm 2$ & $2 \pm 1$ & $2 \pm 1$ \\
\hline
\end{tabular}

Table 4: Phantom Experiment Displacement Error of Virtual Object Placement with Respect to Real Object

\begin{tabular}{|c|c|c|c|}
\hline & $\mathrm{X}$ Axis $\left(^{\circ}\right)$ & Y Axis $\left(^{\circ}\right)$ & $\mathrm{Z}$ Axis $\left(^{\circ}\right)$ \\
\hline Tap to Place & $5 \pm 5$ & $6 \pm 6$ & $7 \pm 6$ \\
\hline $\begin{array}{c}\text { 3-Point } \\
\text { Correspondence }\end{array}$ & $14 \pm 13$ & $17 \pm 20$ & $19 \pm 12$ \\
\hline Keyboard Placement & $7 \pm 5$ & $5 \pm 4$ & $5 \pm 4$ \\
\hline
\end{tabular}

Table 5: Phantom Experiment Rotational Error of Virtual Object Placement with Respect to Real Object

\begin{tabular}{|c|c|c|c|}
\hline & XY Plane (mm) & YZ Plane (mm) & XZ Plane (mm) \\
\hline Tap to Place & $13 \pm 8$ & $12 \pm 9$ & $11 \pm 9$ \\
\hline $\begin{array}{c}\text { 3-Point } \\
\text { Correspondence }\end{array}$ & $8 \pm 5$ & $11 \pm 8$ & $8 \pm 5$ \\
\hline Keyboard Placement & $7 \pm 5$ & $4 \pm 4$ & $5 \pm 3$ \\
\hline
\end{tabular}

Table 6: Clinical Experiment Displacement Error of Virtual Object Placement with Respect to Real Object 


\begin{tabular}{|c|c|c|c|}
\hline & $\mathbf{X ~ A x i s ~}^{\left({ }^{\circ}\right.}$ & Y Axis $\left.\mathbf{(}^{\circ}\right)$ & Z Axis $\mathbf{(}^{\circ}$ ) \\
\hline Tap to Place & $14 \pm 12$ & $12 \pm 11$ & $11 \pm 7$ \\
\hline $\begin{array}{c}\text { 3-Point } \\
\text { Correspondence }\end{array}$ & $23 \pm 23$ & $19 \pm 17$ & \\
\hline Keyboard Placement & $8 \pm 6$ & $9 \pm 7$ & $10 \pm 8$ \\
\hline
\end{tabular}

Table 7: Clinical Experiment Rotational Error of Virtual Object Placement with Respect to Real Object

\subsubsection{Statistical Analysis}

Prior to any statistical tests, we filter the data based on normalized Z scores. Z scores assign a number of each measurement based on how different the specific number is based on standard deviation and the mean of the entire group. Numbers that obtain a $\mathrm{Z}$ score which is greater than 2.68 or less than -2.68 are removed as they are considered the outliers of the data.

Using two statistical tests, we aim to determine which of the methods is most accurate and least accurate for virtual object placement. Initially, we carry out six single-factor ANalysis Of Variance (ANOVA) tests. One for each of the different degrees of freedom (displacement error in the XY, YZ, and XZ planes as well as rotational error about the $\mathrm{X}, \mathrm{Y}$ and $\mathrm{Z}$ axes). In each ANOVA, there are three groups being compared which are the types of placement methods (tap to place, 3-point correspondence, and keyboard placements). The ANOVA test confirms that the average between the three groups is different. From here, we can perform a Tukey test which determines where the difference in means between the three groups is specifically.

After these tests, it was found that the Keyboard method was the most effective, followed by the tap to place, and finally, the 3-point correspondence in the phantom data for both the displacement and rotational errors. Interestingly, for the phantom data, the same trend appears in clinical data regarding the rotational error, but not necessarily for the displacement error. In the latter set of displacement data, the keyboard method is still the best virtual object placement strategy, followed by 3-point correspondence and finally the tap to place method.

\subsubsection{Practical Interpretation and Limitations of the Study}

One of the important things to note about the differences between methods is inherently regarding the algorithms themselves. The way in which these methods manipulate objects is important to determine why some methods for virtual object placement may be more accurate than others.

Tap to place is highly dependent on the resolution of the spatial mapping capabilities of the HoloLens. The highest possible density of mapping triangles is 1300 triangles per cubic meter [59]. Another 
limitation of the tap to place method is that the rotation of the virtual head was fixed during placement. In the event of a real surgery, the patient's head/spine may be at an angle during set up. The inability to change the rotation of the virtual object in real time can be impractical because the orientation of the patient is not necessarily always known well ahead of time. When the user air taps on the virtual object to attach it to the user's world cursor position, the virtual object automatically adjusts to the default rotation of the object's coordinate systems. This inability to rotate the object upon air tapping can be impractical if the orientation of the real-world object is unknown.

3-point placement is also dependent on the resolution of spatial mapping when the points are being chosen on the real object. 3-point correspondence is also heavily based on the placement ability found in the tap to place method as this is how the points are chosen in the real world. The limitations in tap to place based on the mapping resolution are also found in the 3-point placement method. One benefit of this method is that the rotation is automatically handled after the points are chosen by the user. After the three real points are determined manually, the rotation of the virtual plane is then made to match the rotation of the real plane. The rotation of each plane is defined by the normal vectors as explained previously.

The keyboard method was created such that it would only be minimally dependent on the spatial mapping capabilities. Rather than using the entire mesh to place the virtual object, there was only one data point which was available for the spatial understanding of this application. As mentioned previously, when an application is launched, the origin of the room is the location of the HoloLens upon start up. Since the keyboard method does not initialize spatial mapping, only the start-up location is used to establish the axes for the virtual object to move upon. Rather than spatial resolution, the limitation for placement is the sensitivity of the keyboard clicking (in this case, it was $5 \mathrm{~mm}$ ). However, lack of a spatial mesh does pose some caveats. Without a full understanding of the physical room, the holograms are more prone to drift and jitter as they are moved and placed within the room. The mesh provides additional spatial landmarks so that the holograms have anchors to lock to. The drift and jitter were especially apparent in objects which have a great amount of detail. For this reason, the 3D models required down sampling to mitigate the GPU usage and lessen the possibility of crashing the application

Regarding the clinical data we see that there is a large change in accuracy in all the placement methods in all directions. The main difficulty involved with the operating room environment is once again, the operating room workflow. During the phantom experiments, users could take as much time as they would need to place the virtual object on the phantom. In the operating room, it is important that the researchers do not alter the operating room workflow or do anything that could potentially affect patient outcomes during surgery. Users in the operating room would only have about 5 minutes to complete the placementthe only time slot available to take measurements would be after the patient is put to sleep (to ensure that 
the patient is no longer moving), and prior to the draping of the patient. Surgical drapes are required to ensure a sterile environment and cover the remaining areas of the patient that are not required for surgery. After the drapes are placed onto the patient, users can no longer take measurements as the physical patient is no longer visible and no images can be taken.

Another cause for error, specifically for the tap to place and 3-point correspondence, is the constantly changing operating room environment. Operating room personnel walking and moving prior to the surgery to set up all appropriate equipment, moving sterile tools, and other large equipment all contribute to a changing spatial map which is interpreted by the HoloLens. Since the HoloLens is constantly updating its map, it believes that the operating room landscape is constantly shifting- including the main target of the placement interpreted through human input.

It is known that the registration accuracy for neurosurgery must be accurate to the submillimeter level. This level of registration accuracy is seen in all commercially available surgical navigation systems [70]. From these experiments, we see that this is not feasible given many methods of virtual object placement. However, there is still the possibility of exploring the use in presurgical planning for brain surgery. The gold standard for cranial navigation is frame-based stereotactic localization. Stereotactic surgery is a minimally invasive form of surgery used to localize subsurface structures based on a coordinate system. This practice is commonly used for brain surgery. The 3D error for this surgery ranges from 2.5-3.5 mm for both phantom and in-vivo experiments [37][71][72]. The 3D error for the keyboard method proposed here for the phantom experiment is $3.8 \mathrm{~mm}$, which is too high for cranial navigation. However, since the error is quite close to the upper limit of the acceptable limit, there is some potential for use elsewhere in the operating room workflow. Although the mixed reality view is not reliable enough for surgical guidance, surgeons may be able to use it to determine other information such as the location of the first cut and how to best proceed with the surgery given the known information provided by the medical imaging represented in the AR view. The operating room workflow is generally an unforgiving place regarding the development of novel medical devices, especially when considering the human factors guidelines mentioned earlier. In high stakes scenarios, it is common for many devices to be seldomly used despite the potential benefits they have to offer. Although AR does not have a place in the OR for guidance, the ability to display the overlays for potential surgical guidance does show substantial promise for future initiatives regarding augmented reality operating room technologies. 


\section{CONCLUSION}

\subsection{Goals and Findings}

Medical Augmented Reality (AR) is often visualized in movies as "x-ray vision", and other extraordinary capabilities that augment the current state of human vision. As interesting as $\mathrm{x}$-ray vision would be, the goal is to determine proper use cases and methods in which this technology can assist medical personnel. The focus of this thesis is the usability of in the operating room. The question of "is augmented reality a tool or toy?" is a frequent thought. Determining usefulness is directly linked to the field of human computer interaction (HCI). Usability is a large problem that augmented reality faces in all aspects whether as an entertainment system in your living room, or as an assistant to surgeons in the operating room. By simplifying our use cases to neurosurgery, the user base becomes a specific group of people with similar experience and expertise. Neurosurgeons are the main user group and are the target audience for the user-centred design of an AR head mounted display (HMD).

Many concepts from the field of HCI are not only applicable in the practice of designing visual interfaces of common technologies, but also extend to newer technologies such as augmented reality. These HCI practices for developing existing interfaces should also be carried over through the progression of new technologies to maintain continuity for users. Human cognitive behavior is largely the motivation for much of the design choices of modern AR technologies, as well as the various tests carried out in this thesis. In this thesis, we aim to understand the progressive pathway to introducing AR in the operating room, how it can benefit surgeons during stressful scenarios, and determine the level of accuracy delivered by current AR HMDs.

A gradual path to introduce new technologies is crucial for acceptance into the operating room workflow. As such, existing surgical navigation systems which are already incorporated into the operating room workflow are an interesting place to begin. Although not widely accepted for spine surgery, they are frequently used during brain surgery. The workflow for surgical navigation systems, specifically for spine surgery, is considered sub-optimal to many surgeons because of the time consumption and unintuitive interface. One large disconnect in the workflow is when surgeons are required to remember the correct trajectory without the help of surgical guidance for some procedures such as pedicle screw insertion. By superimposing a line directly onto the surgical site, the user is no longer required to remember and recreate the position of the tool during guidance. Surgeons would then be able to trace the trajectory outlined on the surgical field and prevent incorrect screw insertions. In addition, creating a "road map" indicating the safe zones can serve as additional guidance. 
By comparing the angles made by the desired trajectory on the CT scans and the angles made by the surgeon holding the tool directly in the surgical site, there is a magnification effect. Angles created on the surgical site seen through the overhead cameras are statistically larger than the angles seen in the CT images of the patient. This magnification effect also notifies us that the safe zones perceived on the surgical site can appear larger than those on the patient imaging. The magnification effect also produces a visual disconnect while using surgical navigation and creates a large amount of display inertia. The second finding of the angle study confirms that the size of the safe zones increases with the size of the pedicle and further reinforces what we know about C-spine surgery. The tiny pedicles can add difficulty compared to L-spine surgery as there is a smaller margin of safety. This low-level AR integration produces improvement to OR workflow as well as a small introduction to AR in the operating room.

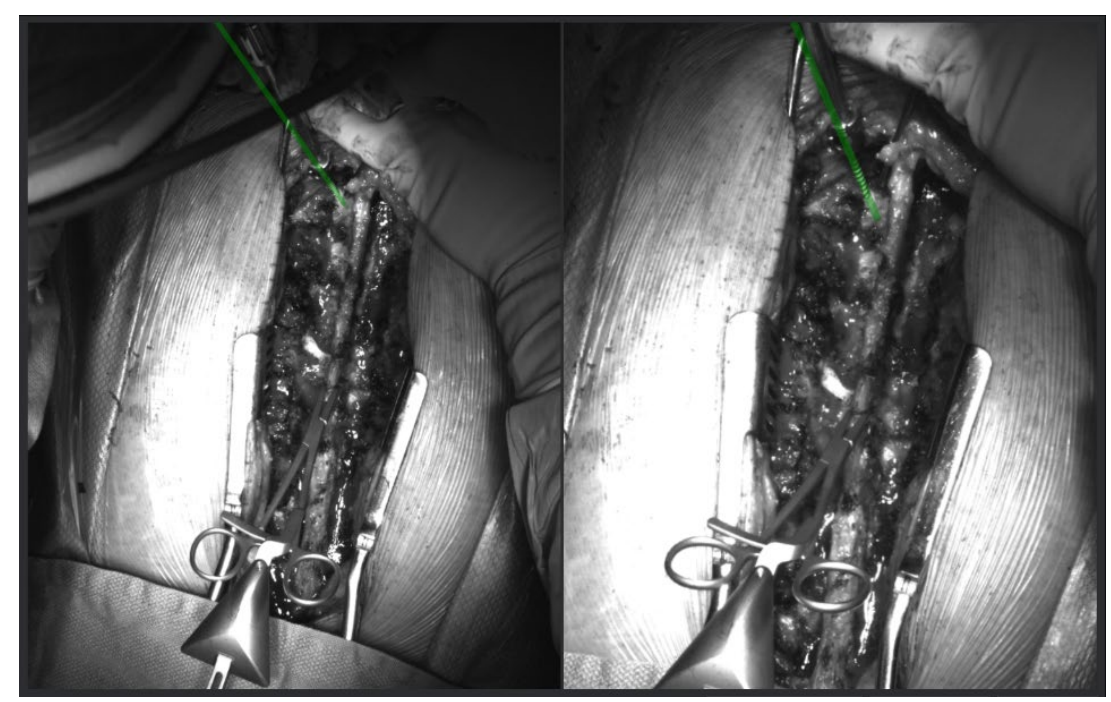

Figure 25: Left and Right camera overhead view of the surgical site during T6 and T7 fusion. The green line imposed onto the surgical site is the ideal pedicle screw trajectory. The screwdriver being used here is untracked and following the pedicle screw.

User-centred design is a crucial component for most HCI practices. Over time, this concept is widely accepted when analyzing previous technologies such as the progression of the Windows operating systems. Large changes between versions can deter user adoption even if the changes are beneficial to the user. In both AR interfaces and conventional interface design, multiple usability tests are required to determine what the main user group requires while completing various tasks. We have seen in other industries such as aviation where AR was implemented effectively for better performance under high risk operations which lead to an overall decrease in cognitive load when the pilots became further accustomed to using the AR HUD (Heads Up Display). To understand what would be useful for a surgeon to view 
during surgery, a usability test was completed with participants of varying skill level. The results were aligned with our hypothesis as users preferred the colours and opacities seen in imaging modalities such as CT. Users with greater skill levels that were used to reading CT images for presurgical planning preferred bright white colours for bone and white for soft tissue.

Another contribution of this thesis was to determine the opacity levels that were considered helpful for the participants. Most studies did not account for the varying levels of opacity and only considered three options: no overlay, solid overlay, and a wireframe model. Here, we aim to evaluate a spectrum of opacities as they change in front of the participants' eyes in real time. Overall, users preferred $95 \%$ opacity for hard tissue and $27 \%$ opacity for surrounding soft tissues.

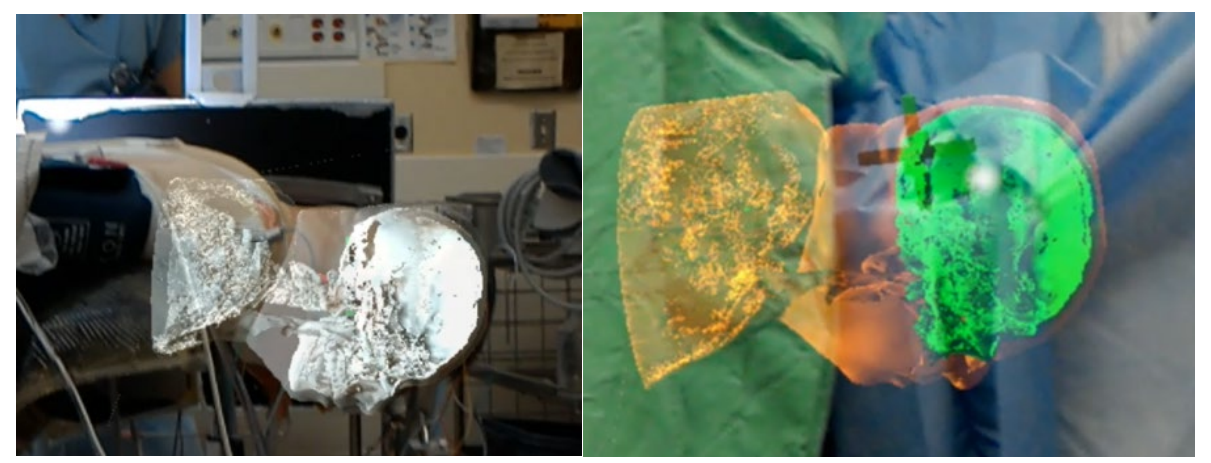

Figures 26: Left image - Ideal representation for surgeons with experience greater than PGY3. Right image Example of contrasting colours for individuals with experience less than PGY3.

Although HCI is incredibly important during the device development stage, the most important factor is accuracy. Using the Microsoft HoloLens, users have the capability to place virtual objects within the room because of the use of spatial mapping. Many research groups have been searching for a method to test the registration accuracy of virtual anatomy to the real patient anatomy to determine whether it can be used for surgical guidance. Many of these studies provide measures for specific placement methods under specific conditions. Our contribution to this body of work is to measure the capability for multiple placement methods using a new method to characterize accuracy between the real and virtual world. The results of phantom experiments were compared to the same tests completed in a clinical environment. During the phantom experiments, it was found that the methods relying on spatial mapping were the less accurate than the manual method of placement using a keyboard at a set sensitivity. During clinical tests, the same accuracy could not be replicated because of the operating room workflow and time constraints. It is important to note that the operating room workflow does not allow for additional time to complete the placement tests as it may interfere with patient safety. Not only does this work confirm that the 
accuracy achieved through the HoloLens is only capable to the extent of presurgical planning, but also displays the importance of the operating room workflow. Due to the fast-paced nature of the operating room, there are incredibly high expectations placed on medical devices. Not only are they expected to be accurate, but must also be capable of seamless integration into the existing operating room and its current technologies.

Our overall hypothesis mentioned earlier is the following: Augmented reality can be deployed in the operating room through human factors considerations seen above in order to avoid the pitfall of slow adoption seen in neurosurgical navigation systems for spine surgery. For a higher likelihood for widespread adoption, we propose an evolutionary pathway for adoption seen below.

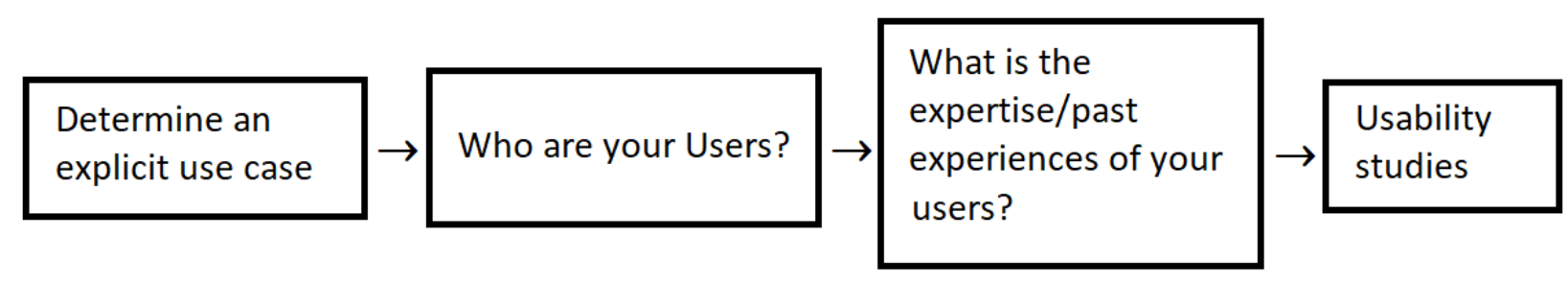

Based on the results of each of the components of the thesis, we see that our hypothesis is confirmed. In the first part of the thesis, we create an AR experience for users of all different expertise for pedicle screw insertion. Users who may not have experience with AR or navigation are more compatible with this hybrid system in comparison to an abrupt introduction to AR in the OR. We demonstrate the difficulty that users experience with conventional navigation and how AR can be used to assist with the workflow for pedicle screw insertion. AR in the context of pedicle screw insertion assists with the workflow by improving cognitive directness, lessening the dependence of human memory, as well as increase the level of display inertia.

For the second and third parts of the thesis, we see deploy usability tests for both the surgeons and the operating room workflow when AR is introduced. Based on the idea of cognitive directness, we see that users prefer viewing AR anatomy which is most analogous to their most commonly used imaging modality. In the final part of the thesis, we test if the Microsoft HoloLens has the necessary accuracy for surgical guidance. This overall registration accuracy ultimately determines how useful of a tool this system can be in the context of surgical guidance. Although the HoloLens is not capable of surgical guidance, it has potential in presurgical planning. Augmented reality technologies can be deployed in the operating room in many ways and have been seen to be effective from many human factors' perspectives. 


\subsection{Future Work}

Existing literature and current events show that there has been extensive work with regards to incorporating AR into the medical field. The field of medical AR is very broad and can have numerous applications in various subspecialties such as otolaryngology, orthopedics, neurosurgery, interventional radiology, sonography, etc. There is a large ambition in the research and industrial communities to develop fully integrated AR HMDs into medicine. Accuracy is among one of the most important goals for medical device development in general. With lives at stake during surgery, or other forms of intervention, accuracy is needed for maintaining a high standard for overall patient care. Although there are many systems which satisfy the accuracy criteria, these new groundbreaking technologies are at risk of poor adoption rates in the operating room. One of the most interesting examples of these circumstances is neurosurgical navigation specifically for spine surgery. As outlined in earlier chapters, there are a number of reasons why surgeons may opt to not use surgical navigation for spine surgery when taking the user interface guidelines and general rules for human factors into consideration.

With the findings presented in this thesis, there is a very distinct future direction. The design of a surgeoncentred AR HMD for neurosurgery is the next step for this research. By creating a pathway to foster the adoption of medical AR technologies into the operating room, a custom surgeon-centred design that integrates well into the operating room workflow has greater promise for widespread use. This overall pathway for the development of an AR HMD consists of the introduction of AR in existing technologies, followed by specific use case studies and usability testing, and finally the implementation of a system which is accurate and precise enough for surgery. Before this can begin, a more rigourous study of surgeon practice and thought must be examined with thorough studies similar to those completed historically in aviation, including eye tracking, tactile feedback testing, hand placement and tracking, and ergonomic studies, among others. These steps lay the foundation for building a completely new headset with a specific purpose rather than attempting to readapt commercially available yet unoptimized systems for the operating room.

Augmented reality is an exciting technology which has an unprecedented potential. With the use of augmented reality, we can elevate patient care to an entirely new level. Patients would see the benefits presented in minimally invasive surgeries without the complications of increased operating times and additional anesthesia. As the technology begins to mature and improve with new advances in the field, the future for a surgeon-centred head mounted display in the operating room looks bright. 


\section{RESEARCH STUDY PROTOCOL}

\section{Title:}

User evaluation and placement accuracy quantification for the visualization of virtual intraoperative anatomy in cranial and spinal neurosurgical procedures

\section{Principal Investigator:}

Dr. Victor Yang Brain Sciences Program, Neurosurgery, Sunnybrook Health Sciences Centre, University of Toronto

\section{Hypotheses and Research Questions:}

We hypothesize that augmented reality headsets - that is, head mounted systems with see-through displays, have the potential to allow for better visualization of anatomy for pre-surgical planning and intraoperative guidance, than traditional screen-based systems, as well as improve surgical workflow and ergonomics while lowering risk. With this study specifically, we wish to examine how users perceive the appearance of 3-Dimensional anatomical projections in an intraoperative setting, and to quantify the placement accuracy of these projections with respect to patient anatomy. In this, our specific research questions are:

1. What are the best methods for visualization of 3-Dimensional anatomical models on patient anatomy according to knowledge users, with respect to model colour, opacity, displayed anatomy, and time for placement.

2. What is the placement accuracy and error when placing these models on patient anatomy in an intraoperative setting.

Question 1 is a qualitative study that relies on user feedback, while question 2 will provide a quantitative measure for placement accuracy.

\section{Background:}

Surgical navigation has become the standard-of-care in cranial neurosurgery for the localization of subsurface structures, including neoplasms and vascular lesions, and for targeting of electrical implants to specific nuclei. A similar evolution has occurred in navigation for spinal surgery in the past decade driven mainly by the number of procedures performed, with 410,000 spinal fusions performed in the United States in 2008 and this number expected to rise significantly in the coming decades due to the aging population. Moreover, Minimally invasive surgeries (MIS) are more often being employed as they have shown to reduce blood loss, operative times, and hospital length-of-stay for patients, relative to open surgery. ${ }^{1-7}$

As MIS procedures become standard, visualization of underlying anatomy becomes more difficult. While navigation systems do allow for the visualization of underlying 
structures, current systems employ screens located away from the surgical exposure, allowing for potential instrumentation errors when looking at the navigation screen based on tool tip placement, and deviations from the navigated trajectory when the surgeon returns to the exposure. Moreover, the lack of exposed anatomical landmarks can make it difficult for surgeons to visualize underlying anatomy.

A potential solution that improves both workflow and visualization of underlying anatomy is to use 3-Dimensional anatomical overlays on augmented reality head-mounted displays (AR-HMDs) to allow for visualization of subsurface structures (Fig. 1). Augmented Reality (AR) systems for pre-surgical planning and intraoperative guidance have been study to some degree in the past, but have done little to quantify the absolute placement error, and often worked using a separate screen or tablet device, rendering them of little use in the operating room. Moreover, any AR-HMD must overcome other potential problems related to user interaction, including visual fatigue, relative depth perception of models, occlusion, delays and lag in displayed models, and inattentional blindness caused by increased cognitive load..$^{8-16}$

\section{Purpose}

Our research group has developed a pipeline for the creation of virtual patientspecific anatomies with varied methods for visualization and matching to real anatomy using off-the-shelf AR Headsets. The purpose of this study is to assess the quality of the matched virtual anatomy through user feedback, and to assess the accuracy of various matching techniques in the operating room setting.

\section{Review of Work to Date:}

Our group has developed a pipeline for semi-automatic segmentation of CT and MR data to allow for models of the full head/body, skull/bony anatomy, and underlying large tissue structures (i.e. tumours) to be visualized (Fig. 2). These models can be displayed with varying colours and transparencies, and uploaded to an AR headset for deployment. The models, when deployed, are then matched using one of 4 current matching algorithms.

To date, the model matching algorithm and method has been tested on a spine and cranial phantom (Fig. 3), and appeared to allow for adequate visualization at a level of accuracy sufficient for pre-surgical planning. 

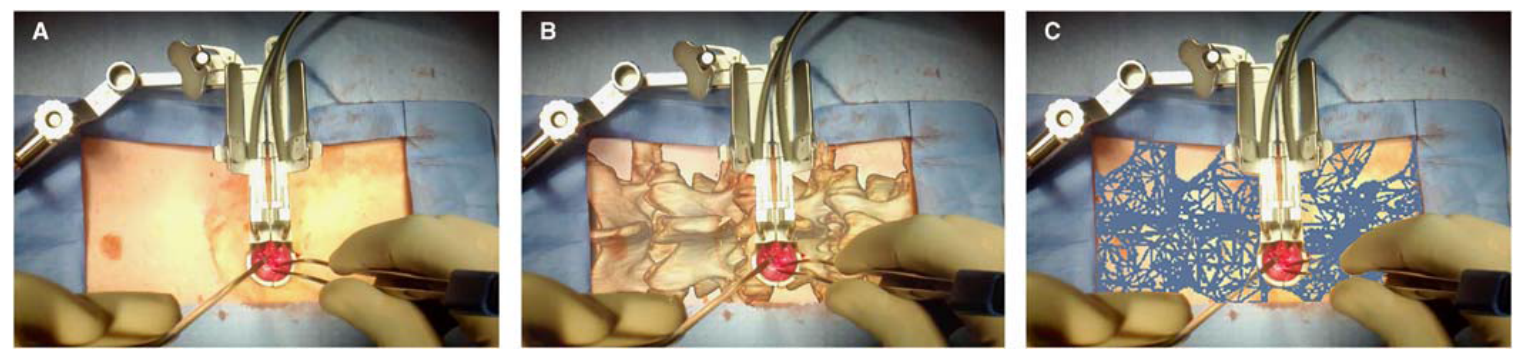

Fig. 1: A rendering of an augmented reality overlay displaying spine anatomy below the surface during a MIS spine procedure.

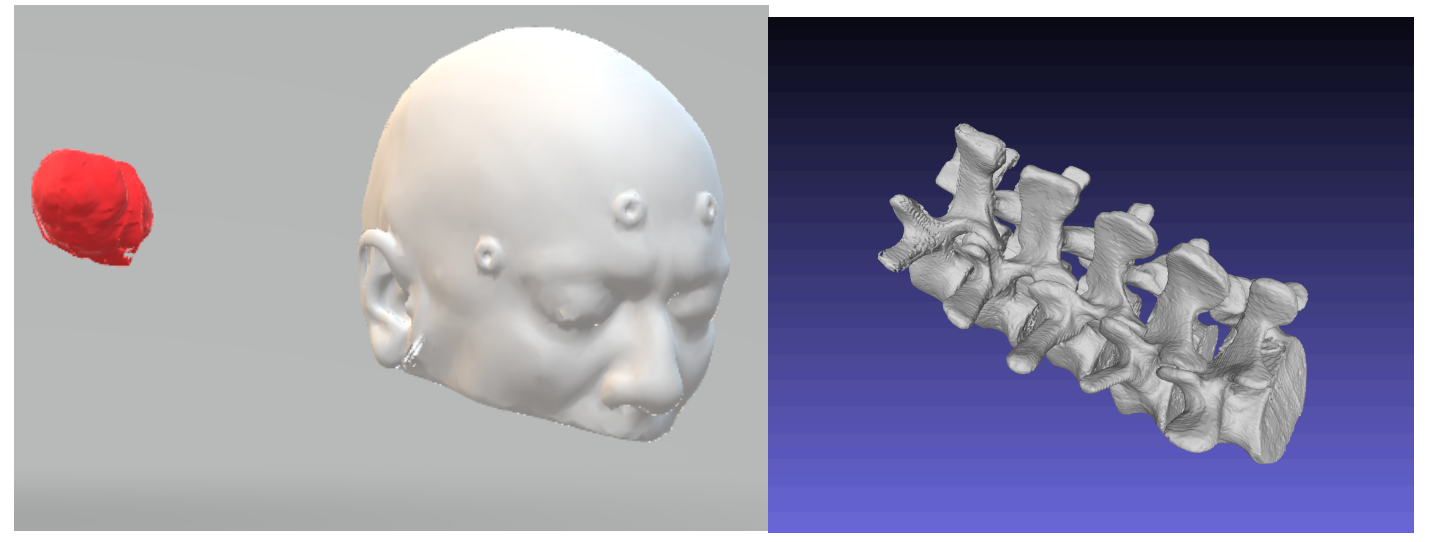

Fig. 2: Example of a segmented and rendered (left) tumour and (centre) head, to scale, as well as (right) spine (bony anatomy) 


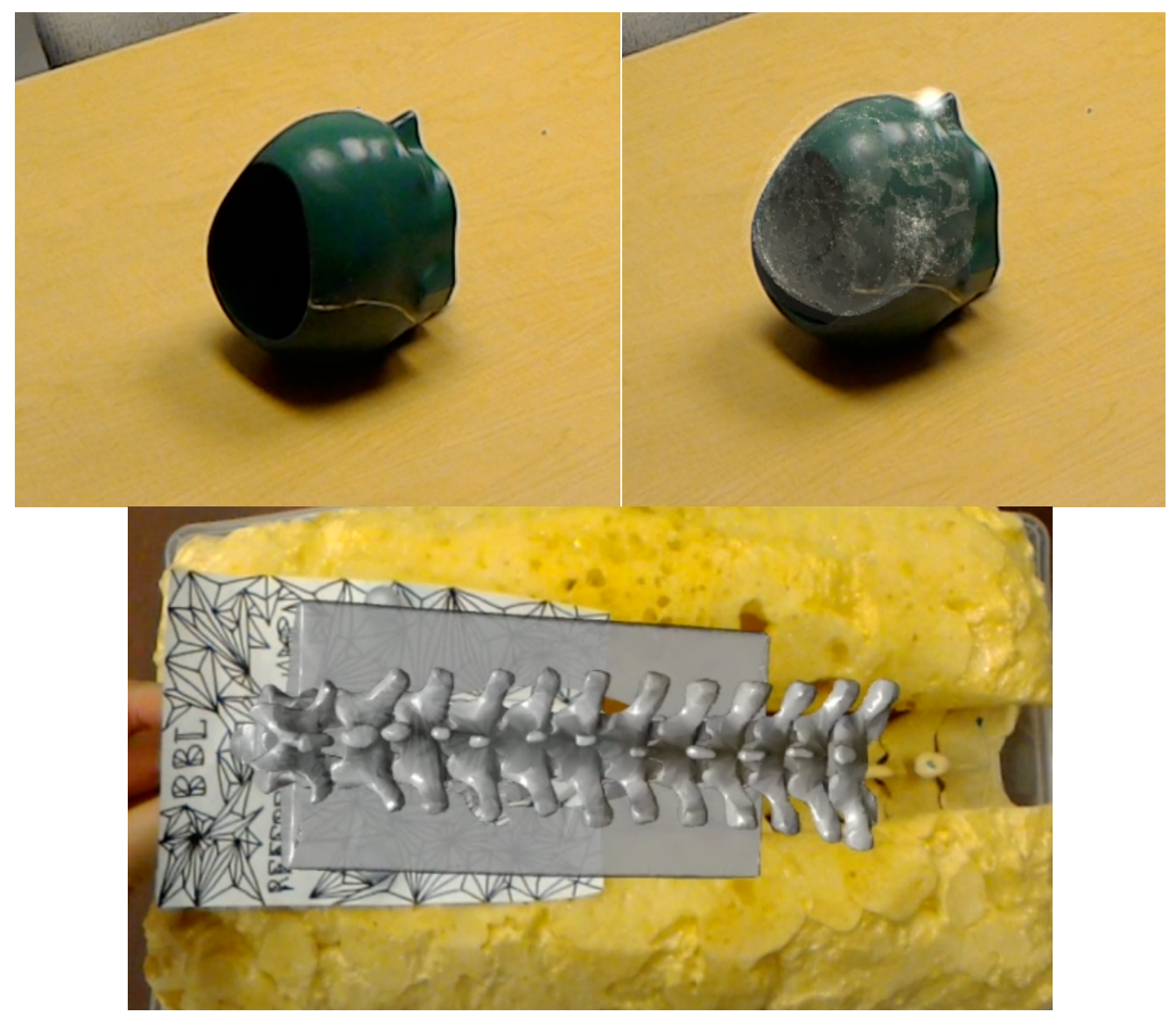

Fig. 3: (Left) a head phantom and (Right) the same phantom with head overlayed, and (bottom) a spine matched to a spine phantom using one of the four matching methods.
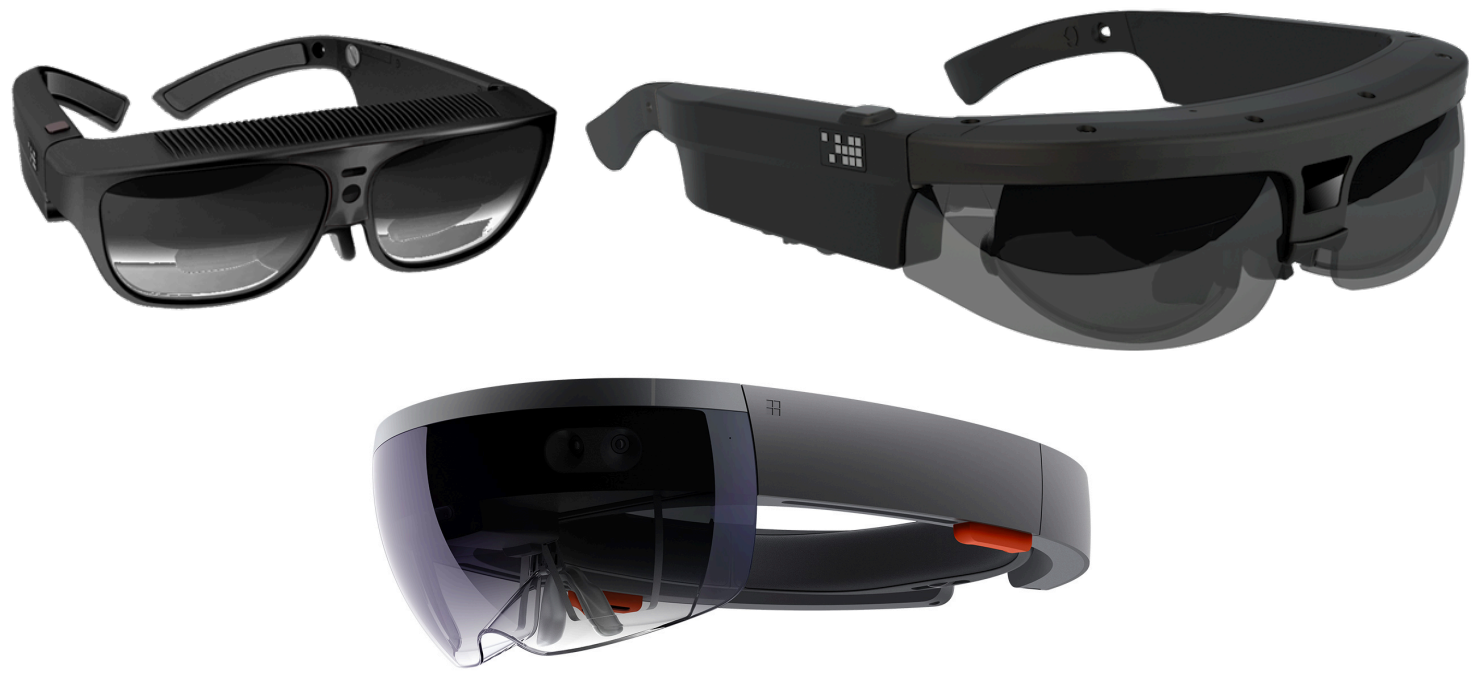

Fig. 4: The (Top left) Osterhout Design Group R7, (top right) Osterhout design group R9, and (bottom) Microscoft HoloLens Augmented Reality headsets which will be employed in this study. 


\section{Methods:}

This is an in vivo human study aimed to assess the feasibility of an augmentedreality based system for the visualization of underlying patient anatomy during cranial and spinal surgical procedures. The models generated will be evaluated based on user experience.

The inclusion criteria are:

a) Greater than 18 years of age, able to provide consent, or has substitute decision maker available to consent.

b) Scheduled to undergo some form of cranial or spinal procedure with CT or MR imaging scheduled as per standard-of-care. Specifically, the patient is scheduled to undergo any of the following procedures:

- Neurosurgical cranial: supratentorial and infratentorial craniotomies for primary or metastatic brain tumours, artervienous malformations, or dural arteriovenous fistula.

- Neurosurgical peripheral: peripheral nerve decompression (entrapment neuropathy) or peripheral nerve sheath tumours.

- Spinal: laminectomy for extradural or intradural tumours (primary or metastatic); laminectomy and fusion for spinal fracture, stenosis, or disk herniation; laminectomy and disconnection of spinal dural arterivenous fistula.

Patients will typically have had a pre-operative CT or MRI as standard of care in order to allow a treatment decision to be made and for navigation to occur. They will typically not require any additional pre- or post-operative imaging, and no additional imaging will be required for study purposes.

All procedures will be performed by a staff neurosurgeon at Sunnybrook (Dr. Victor Yang), with the assistance of one or more trainees and/or staff, as per standard of care.

\section{Model generation and upload to AR headset}

After receiving consent for patient participation in the study, CT and/or MRI data will be anonymized and placed on an encrypted computer located at the hospital. The imaging data will then be segmented to extract the full anatomy surface (i.e skin surface, full head, etc.), bony anatomy (i.e skull and/or spine), and underlying tissues of interest (i.e tumour). The models will be checked by the research group, texturing (i.e colouring, transparency, etc.) will be added, and the model will be uploaded to one of three AR headsets (Microsoft HoloLens, Osterhout Design Group R7, Osterhout Design Group R9, as shown in Fig. 4).

\section{Surgical procedure}


For this study, surgical procedures will not be altered in any way and will be performed according to the established standard-of-care. Moreover, this study is purely for the visualization of the model overlays to inform later augmented reality system design, but will NOT be used for any clinical decision making at any point during the procedure.

Before beginning exposure at the surgical site, the patient is prepped by sterilizing the surgical site. This is typically done by one of surgical staff or trainees, while the other remains "unscrubbed", or unsterile. At this time, the research staff will match the virtual anatomy to the patient anatomy using one of four methods (manual gesture or keyboard placement, automatic point picking, or automatic tracking). Once placed, the unscrubbed surgeon will observe the visualization and the research staff will note feedback given from the surgeon, as described in the data collection form. Once notes have been provided, the surgeon will continue with the procedure as normal, and recordings will be taken from multiple points of view so that the accuracy can be later quantified after the surgery. This same matching procedure will be conducted again at closing.

\section{Sample size calculation}

Since this is the first in vivo examination of these visualization and matching methods in neurosurgical procedures, no data in literature is available to guide a sample size calculations. However, based on the anticipated volume of each category, sample sizes of 35 cranial and 35 spinal patients were chosen in order to achieve an effective size of $0.5(\alpha=0.05, \beta=0.80){ }^{17}$ Peripheral nerve procedures are performed infrequently, typically one or two cases per month (unpublished data from the Sunnybrook Health Sciences Centre Division of Neurosurgery), hence the majority of the sample size will come from cranial and spinal procedures.

\section{Data Collection:}

Data will be collected during each procedure in the form of on-board video recordings from the AR headsets. These recordings will be used to measure translational and rotational error from the positioning methods employed.

Immediately following the procedure, the surgeon and any involved trainees will be asked to rate (on a 5-point Likert scale) a number of parameters based on the visualized anatomy (see Data Collection Form). These parameters include:

- Model resolution

- Model colouration

- Model transparency

- Model layers

- Update lag and delay

- Ergonomics/comfort 
A still image of the overlay will be taken (without any patient-identifying information), for comparison purposes for raters, as a memory-refreshing tool after the procedure is completed.

\section{Risks:}

There are no relevant risks to this study as there are no changes to the standard-ofcare. No additional pre- or post-operative imaging is required for the study. There will be a maximum of 5 minutes added to the procedure time as a result of the study, which will not impact any patient or hospital outcomes.

\section{Benefits:}

There are no immediate benefits as patients enrolled will receive standard of care for their procedures.

\section{Timeline:}

This research will take approximately 6 months to complete. The expected start date is April 1, 2018 and the estimated end date is October 1, 2018. After the study and the data has been compiled and analyzed, these results will be used to inform further AR system design. 


\section{Data Analysis:}

Primary data analysis to determine placement accuracy involves the acquired onboard AR recordings of the model overlays mentioned above. Multiple morphological features on the models will be compared to their related features on patient anatomy to determine absolute translational and rotational error in each axis.

With regards to user-feedback, quantitative metrics will include mean ratings in each of the included parameters (see Data Collection Form). Qualitative feedback will also be collected from each rater.

Data analysis will be performed at Sunnybrook Health Sciences Centre.

\section{Implications of Research:}

The research conducted will demonstrate the feasibility of model overlays using an augmented reality headset, as well as demonstrate that a sufficient placement accuracy can be achieved for pre-surgical planning in neurosurgical procedures, and possibly for intraoperative procedures. This research will guide further improvements and streamlining of this technique for the neurosurgical operating room, and has implications that can be spread to other types of surgery, including orthopaedic and general surgery. With subsequent design iterations, it is expected that this technology can be used to visualize anatomy for pre-surgical planning, and eventually act as a standalone intraoperative navigation system for surgeons, with an overall improvement to operating room workflow, ergonomics, and safety in the operating room.

\section{References:}

1. Ciol M, Deyo RA, Howell E, Kreif $S$. An assessment of surgery for spinal stenosis: time trends, geographic variations, complications, and reoperations, J Am Geriatr Soc 1996, 44: 285-90.

2. Rajee S, Bae H, Knim L, Delamarter R. Spinal fusion in the United States: analysis of trends from 1998 to 2009. Spine (Phila. Pa 1976) 2012.

3. Ahn SS, Kim SH, Kim DW, Lee BH. Comparison of outcomes of percutaneous endoscopic lumbar discectomy and open lumbar microdiscectomy for young adults: a retrospective matched cohort study. World Neurosurg 2016; 86: 250-8.

4. Hubbe U, Franco-Jimenez P, Klingler JH, Vasilikos I, Scholz C, Kogias E. Minimally invasive tubular microdiscectomy for recurrent lumbar disc herniation. $J$ Neurosurg Spine 2016; 24(1): 48-53.

5. Chang X, Chen B, Li HY, Han XB, Zhou Y, Li CQ. The safety and efficacy of minimally invasive discectomy: a meta-analysis of prospective randomized controlled trials. Int Orthop 2014; 38(6): 1225-34. 
6. Tomasino A, Parikh K, Steinberger J, Knopman J, Boockvar J, Hartl R. Tubular microsurgery for lumbar discectomies and laminectomies in obese patients: operative results and outcome. Spine 2009; 34(18): E664-72.

7. MIS Neurosurgery paper....

8. Watt SJ, Akeley K, Ernst MO, Banks MS. Focus cues affect perceived depth. $J$ Vis. 2005;5:834-62.

9. Bando T, lijima A, Yano S. Visual fatigue caused by stereoscopicimages and the search for the requirement to prevent them: a review. Displays. 2012;33:76-83.

10. Nagata S. How to reinforce perception of depth in single two-dimensional pictures. Proc SID. 1983;25:239-46.

11. Hughes-Hallett A, Mayer EK, et al. Inattention blindness in surgery. Surg Endosc. 2015;29:3184-9.

12. Simons DJ, Chabris CF. Gorillas in our midst: sustained inattentional blindness for dynamic events. Perception. 1999;28:1059-74.

13. Dixon BJ, Daly MJ, Chan HH, Vescan A, Witterick IJ, Irish JC. Inattentional blindness increased with augmented reality surgical navigation. Am J Rhinol Allergy. 2014;28:433-7.

14. Dixon BJ, Daly MJ, Chan H, Vescan AD, Witterick IJ, Irish JC. Surgeons blinded by enhanced navigation: the effect of augmented reality on attention. Surg Endosc. 2013;27: 454-61.

15. Marcus HJ, Pratt $P$, Hughes-Hallett $A$, et al. Comparative effectiveness and safety of image guidance systems in surgery: a preclinical randomised study. Lancet. 2015;385:S64.

16. Guha D, Alotaibi NM, Nguyen N, Gupta S, McFaul C, Yang VXD. Augmented Reality in Neurosurgery: A review of current concepts and emerging applications. Can J Neurol Sci. 2017, 44:235-245.

17. F. Faul, E. Erdfelder, A.-G. Lang, A. Buchner and C. Kiel. A Flexible Statistical Power Analysis Program for the Social, Behavioral, and Biomedical Sciences. Behavioral Research Methods. 2007, 39:175-91. 
Title: User evaluation and placement accuracy quantification for the visualization of virtual intraoperative anatomy in cranial and spinal neurosurgical procedures

\section{General Case Information}

\begin{tabular}{|l|l|}
\hline Date & \\
\hline $\begin{array}{l}\text { Study Subject } \\
\text { Identifier (AR1-XX) }\end{array}$ & \\
\hline Surgery type & \\
\hline Headset type & \\
\hline Overlaid anatomy & \\
\hline Transparencies & \\
\hline Colouration & \\
\hline Research personnel & \\
\hline $\begin{array}{l}\text { Time required for } \\
\text { placement }\end{array}$ & \\
\hline $\begin{array}{l}\text { Method of } \\
\text { placement }\end{array}$ & \\
\hline Surgeons & \\
\hline $\begin{array}{l}\text { Amount of time } \\
\text { wearing headset }\end{array}$ & \\
\hline $\begin{array}{l}\text { Times when headset } \\
\text { was worn }\end{array}$ & \\
\hline Surgery start & \\
\hline Surgery end & \\
\hline Notes on procedure & \\
\hline
\end{tabular}

\section{Instructions:}


Please rate the augmented reality system and placement, as well as your experience in it in comparison to standard visualizations from navigation systems and the like.

Rating system is as follows:

1 - AR system significantly worse

2 - AR system slightly worse

3 - AR system is the same

$4-A R$ system slightly better

5 - AR system significantly better

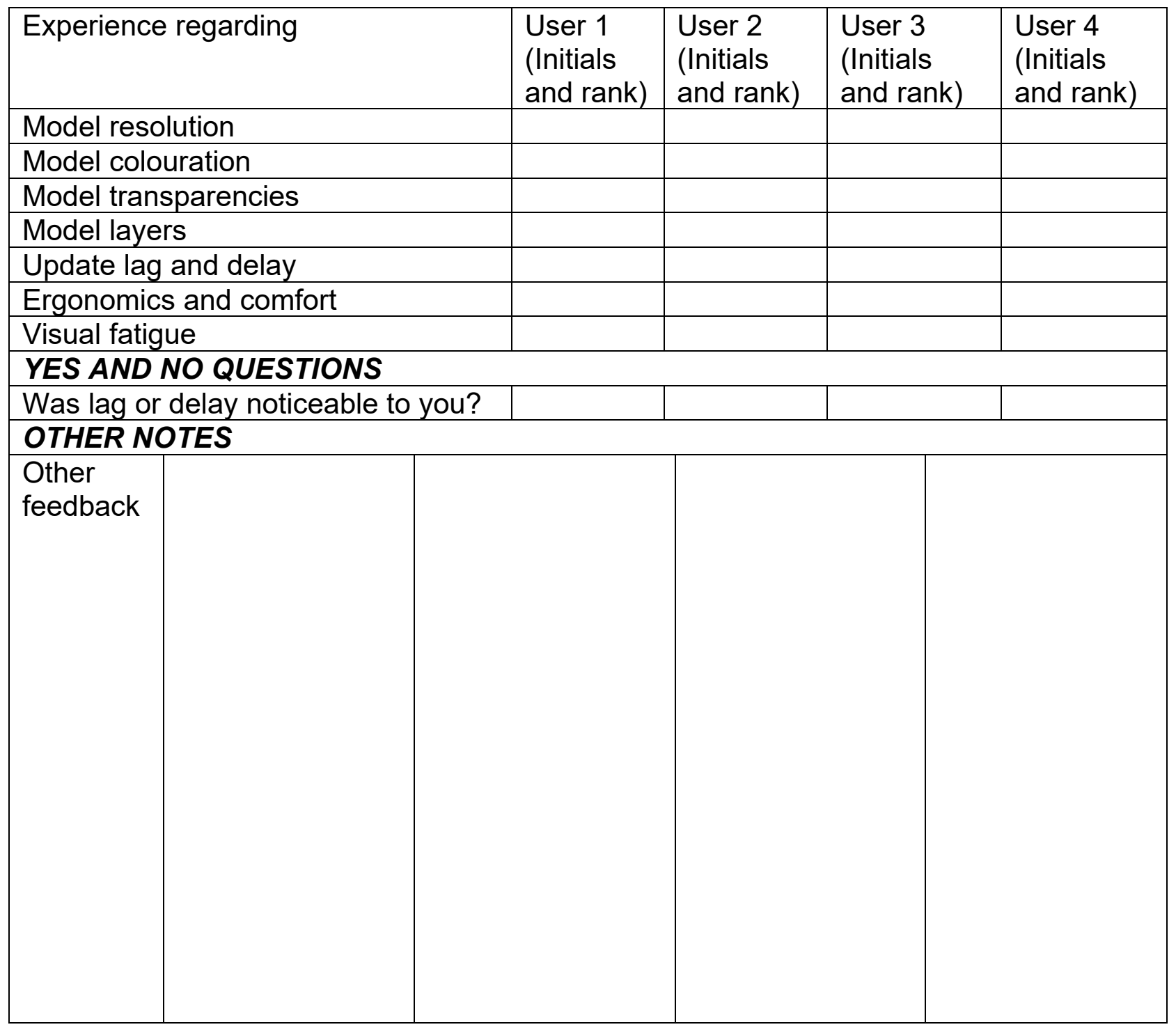




\section{REFERENCES}

[1] R. Härtl, K. S. Lam, J. Wang, A. Korge, F. Kandziora, and L. Audigé, "Worldwide Survey on the Use of Navigation in Spine Surgery," World Neurosurg., vol. 79, no. 1, pp. 162-172, Jan. 2013.

[2] M. Bydon, D. Mathios, M. Macki, R. De la Garza-Ramos, N. Aygun, D. M. Sciubba, T. F. Witham, Z. L. Gokaslan, A. Bydon, and J.-P. Wolinksy, "Accuracy of C2 pedicle screw placement using the anatomic freehand technique," Clin. Neurol. Neurosurg., vol. 125, pp. 24-27, Oct. 2014.

[3] L.-P. Nolte, H. Visarius, E. Arm, F. Langlotz, O. Schwarzenbach, and L. Zamorano, "Computeraided fixation of spinal implants," J. Image Guid. Surg., vol. 1, no. 2, pp. 88-93, 1995.

[4] J. Traub, P. Stefan, S. M. Heining, T. Sielhorst, C. Riquarts, E. Euler, and N. Navab, "LNCS 4190 - Hybrid Navigation Interface for Orthopedic and Trauma Surgery," 2006.

[5] A. P. King, P. J. Edwards, C. R. Maurer Jr., D. A. de Cunha, D. J. Hawkes, D. L. G. Hill, R. P. Gaston, M. R. Fenlon, A. J. Strong, C. L. Chandler, A. Richards, and M. J. Gleeson, "A System for Microscope-Assisted Guided Interventions,” Stereotact. Funct. Neurosurg., vol. 72, no. 2-4, pp. 107-111, 1999.

[6] A. Mason, R. Paulsen, J. M. Babuska, S. Rajpal, S. Burneikiene, E. L. Nelson, and A. T. Villavicencio, "The accuracy of pedicle screw placement using intraoperative image guidance systems," J. Neurosurg. Spine, vol. 20, no. 2, pp. 196-203, Feb. 2014.

[7] P. J. Belmont, W. R. Klemme, A. Dhawan, and D. W. Polly, "In Vivo Accuracy of Thoracic Pedicle Screws," Spine (Phila. Pa. 1976)., vol. 26, no. 21, pp. 2340-2346, Nov. 2001.

[8] O. Schwarzenbach, U. Berlemann, B. Jost, H. Visarius, E. Arm, F. Langlotz, L.-P. Nolte, and C. Ozdoba, "Accuracy of Computer-Assisted Pedicle Screw Placement," Spine (Phila. Pa. 1976)., vol. 22, no. 4, pp. 452-458, Feb. 1997.

[9] S. I. Esses, B. L. Sachs, and V. Dreyzin, "Complications associated with the technique of pedicle screw fixation. A selected survey of ABS members.," Spine (Phila. Pa. 1976)., vol. 18, no. 15, pp. 2231-8; discussion 2238-9, Nov. 1993.

[10] R. Jakubovic, D. Guha, S. Gupta, M. Lu, J. Jivraj, B. A. Standish, M. K. Leung, A. Mariampillai, K. Lee, P. Siegler, P. Skowron, H. Farooq, N. Nguyen, J. Alarcon, R. Deorajh, J. Ramjist, M. Ford, P. Howard, N. Phan, et al., "High Speed, High Density Intraoperative 3D Optical Topographical Imaging with Efficient Registration to MRI and CT for Craniospinal Surgical Navigation.," Sci. Rep., vol. 8, no. 1, p. 14894, Oct. 2018.

[11] C. P. Childers and M. Maggard-Gibbons, "Understanding Costs of Care in the Operating Room," JAMA Surg., vol. 153, no. 4, p. e176233, Apr. 2018.

[12] A. Macario, "What does one minute of operating room time cost?," J. Clin. Anesth., vol. 22, no. 4, pp. 233-236, 2010.

[13] R. D. Shippert, "A Study of Time-Dependent Operating Room Fees and How to save $\$ 100000$ by Using Time-Saving Products,” Am. J. Cosmet. Surg., vol. 22, no. 1, pp. 25-34, Mar. 2005.

[14] D. Hix and H. R. Hartson, Developing user interfaces : ensuring usability through product \&amp; process. New York: J. Wiley, 1993.

[15] E. L. Wiener, "Human Factors of Advanced Technology (\&quot;Glass Cockpit\&quot;) Transport 
Aircraft," 1989.

[16] D. Guha, R. Jakubovic, S. Gupta, N. M. Alotaibi, D. Cadotte, L. B. da Costa, R. George, C. Heyn, P. Howard, A. Kapadia, J. M. Klostranec, N. Phan, G. Tan, T. G. Mainprize, A. Yee, and V. X. D. Yang, "Spinal intraoperative three-dimensional navigation: correlation between clinical and absolute engineering accuracy," Spine J., vol. 17, no. 4, pp. 489-498, 2017.

[17] K. Abhari, J. S. H. Baxter, E. C. S. Chen, A. R. Khan, T. M. Peters, S. De Ribaupierre, and R. Eagleson, "Training for planning tumour resection: Augmented reality and human factors," IEEE Trans. Biomed. Eng., vol. 62, no. 6, pp. 1466-1477, 2015.

[18] "Re: Safety Notice Related to the Use of Non-Medtronic Instruments with the NavLock ${ }^{\mathrm{TM}}$ Tracker," 2017.

[19] S. Andress, A. Johnson, M. Unberath, A. F. Winkler, K. Yu, J. Fotouhi, S. Weidert, G. Osgood, and N. Navab, "On-the-fly augmented reality for orthopedic surgery using a multimodal fiducial," J. Med. Imaging, vol. 5, no. 02, p. 1, Jan. 2018.

[20] P. Markelj, D. Tomaževič, B. Likar, and F. Pernuš, "A review of 3D/2D registration methods for image-guided interventions," Med. Image Anal., vol. 16, pp. 642-661, 2012.

[21] J. Hajek, M. Unberath, J. Fotouhi, B. Bier, S. C. Lee, G. Osgood, A. Maier, M. Armand, and N. Navab, "Closing the Calibration Loop: An Inside-out-tracking Paradigm for Augmented Reality in Orthopedic Surgery."

[22] D. Reisberg, Ed., The Oxford Handbook of Cognitive Psychology. Oxford University Press, 2013.

[23] M. Feuerstein, T. Sielhorst, and N. Navab, “Advanced Medical Displays: A Literature Review of Augmented Reality,” J. Disp. Technol., vol. 4, no. 4, pp. 451-467, 2017.

[24] R. T. Azuma, "A Survey of Augmented Reality,” 1997.

[25] K. A. Guru, E. T. Esfahani, S. J. Raza, R. Bhat, K. Wang, Y. Hammond, G. Wilding, J. O. Peabody, and A. J. Chowriappa, "Cognitive skills assessment during robot-assisted surgery: separating the wheat from the chaff," BJU Int., vol. 115, no. 1, pp. 166-174, Jan. 2015.

[26] D. Guha, N. M. Alotaibi, N. Nguyen, S. Gupta, C. McFaul, and V. X. D. Yang, "Augmented Reality in Neurosurgery: A Review of Current Concepts and Emerging Applications," Can. J. Neurol. Sci. / J. Can. des Sci. Neurol., vol. 44, no. 03, pp. 235-245, May 2017.

[27] C. Chen, J. Wang, K. Li, Y. Liu, and X. Chen, "Visual fatigue caused by watching 3DTV: an fMRI study.," Biomed. Eng. Online, vol. 14 Suppl 1, no. Suppl 1, p. S12, 2015.

[28] H. J. Marcus, P. Pratt, A. Hughes-Hallett, T. P. Cundy, A. P. Marcus, G.-Z. Yang, A. Darzi, and D. Nandi, "Comparative effectiveness and safety of image guidance systems in neurosurgery: a preclinical randomized study.," J. Neurosurg., vol. 123, no. 2, pp. 307-13, Aug. 2015.

[29] S. Nagata, "HOW TO REINFORCE PERCEPTION OF DEPTH IN SINGLE TWODIMENSIONAL PICTURES."

[30] A. Deshpande and I. Kim, "The effects of augmented reality on improving spatial problem solving for object assembly," Adv. Eng. Informatics, vol. 38, pp. 760-775, 2018.

[31] E. R. Velamkayala, M. V. Zambrano, and H. Li, "Effects of HoloLens in Collaboration: A Case in Navigation Tasks," Proc. Hum. Factors Ergon. Soc. Annu. Meet., vol. 61, no. 1, pp. 2110-2114, Sep. 2017. 
[32] A. E. Andersson, I. Bergh, J. Karlsson, B. I. Eriksson, and K. Nilsson, "Traffic flow in the operating room: An explorative and descriptive study on air quality during orthopedic trauma implant surgery," Am. J. Infect. Control, vol. 40, no. 8, pp. 750-755, Oct. 2012.

[33] U. Mezger, C. Jendrewski, and M. Bartels, "Navigation in surgery."

[34] A. Elmi-Terander, H. Skulason, M. Soderman, J. Racadio, R. Homan, D. Babic, N. Van Der Vaart, and R. Nachabe, "Surgical navigation technology based on augmented reality and integrated 3D intraoperative imaging a spine cadaveric feasibility and accuracy study," Spine (Phila. Pa. 1976)., vol. 41, no. 21, pp. E1303-E1311, 2016.

[35] "Desktop Windows Version Market Share Worldwide | StatCounter Global Stats." [Online]. Available: http://gs.statcounter.com/windows-version-market-share/desktop/worldwide/2012. [Accessed: 03-May-2019].

[36] "Desktop Windows Version Market Share Worldwide | StatCounter Global Stats." [Online]. Available: http:/gs.statcounter.com/windows-version-market-share/desktop/worldwide/\#monthly201901-201901-bar. [Accessed: 03-May-2019].

[37] R. Jakubovic, D. Guha, S. Gupta, M. Lu, J. Jivraj, B. A. Standish, M. K. Leung, A. Mariampillai, K. Lee, P. Siegler, P. Skowron, H. Farooq, N. Nguyen, J. Alarcon, R. Deorajh, J. Ramjist, M. Ford, P. Howard, N. Phan, et al., "High Speed, High Density Intraoperative 3D Optical Topographical Imaging with Efficient Registration to MRI and CT for Craniospinal Surgical Navigation," Sci. Rep., vol. 8, no. 1, p. 14894, Dec. 2018.

[38] R. Szeliski, "Computer Vision: Algorithms and Applications,” 2010.

[39] “Spine Vue | Minimally Invasive Spine Surgery Dallas TX.” [Online]. Available: https://spinevuetx.com/minimally-invasive-spine-surgery-dallas/. [Accessed: 31-May-2019].

[40] S. McClelland, III, and J. A. Goldstein, "Minimally Invasive versus Open Spine Surgery: What Does the Best Evidence Tell Us?," J. Neurosci. Rural Pract., vol. 8, no. 2, p. 194, 2017.

[41] I. Kuhlemann, M. Kleemann, P. Jauer, A. Schweikard, and F. Ernst, "Towards X-ray free endovascular interventions - using HoloLens for on-line holographic visualisation," Healthc. Technol. Lett., vol. 4, pp. 184-187, 2017.

[42] A. Soueid, D. Oudit, S. Thiagarajah, and G. Laitung, "The pain of surgery: Pain experienced by surgeons while operating," Int. J. Surg., vol. 8, no. 2, pp. 118-120, Jan. 2010.

[43] C. Karmonik, T. B. Boone, and R. Khavari, "Workflow for Visualization of Neuroimaging Data with an Augmented Reality Device," J. Digit. Imaging, vol. 31, no. 1, pp. 26-31, Feb. 2018.

[44] K. Abhari, J. S. H. Baxter, A. R. Khan, T. M. Peters, R. Eagleson, ; A R Khan, T. M. Peters, D. Ribaupierre, R. Eagleson, and S. De Ribaupierre, "Visual Enhancement of MR Angiography Images to Facilitate Planning of Arteriovenous Malformation Interventions ACM Reference Format," ACM Trans. Appl. Percept., vol. 12, no. 1, 2015.

[45] D. J. Simons and C. F. Chabris, "Gorillas in Our Midst: Sustained Inattentional Blindness for Dynamic Events,” Perception, vol. 28, no. 9, pp. 1059-1074, Sep. 1999.

[46] A. Hughes-Hallett, E. K. Mayer, H. J. Marcus, P. Pratt, S. Mason, A. W. Darzi, and J. A. Vale, "Inattention blindness in surgery," Surg. Endosc., vol. 29, no. 11, pp. 3184-3189, Nov. 2015.

[47] D. Guha, N. M. Alotaibi, N. Nguyen, S. Gupta, C. McFaul, and V. X. D. Yang, "Augmented Reality in Neurosurgery: A Review of Current Concepts and Emerging Applications," Can. J. 
Neurol. Sci. /J. Can. des Sci. Neurol., vol. 44, no. 3, pp. 235-245, May 2017.

[48] S. Fadden, C. Wickens, and P. Ververs, "Costs and benefits of head up displays - An attention perspective and a meta analysis," in 2000 World Aviation Conference, 2000.

[49] B. J. Dixon, M. J. Daly, H. Chan, A. D. Vescan, I. J. Witterick, and J. C. Irish, "Surgeons blinded by enhanced navigation: the effect of augmented reality on attention," Surg. Endosc., vol. 27, no. 2, pp. 454-461, Feb. 2013.

[50] B. J. Dixon, M. J. Daly, H. H. L. Chan, A. Vescan, I. J. Witterick, and J. C. Irish, "Inattentional Blindness Increased with Augmented Reality Surgical Navigation," Am. J. Rhinol. Allergy, vol. 28, no. 5, pp. 433-437, Sep. 2014.

[51] D. M. Hoffman, A. R. Girshick, K. Akeley, and M. S. Banks, "Vergence-accommodation conflicts hinder visual performance and cause visual fatigue," J. Vis., vol. 8, no. 3, p. 33, Mar. 2008 .

[52] A. Kumcu, L. Vermeulen, S. A. Elprama, P. Duysburgh, L. Platiša, Y. Van Nieuwenhove, V. N. Van De Winkel, A. Jacobs, W. Philips, and J. Van Looy, "Effect of video lag on laparoscopic surgery: correlation between performanece and usability at low latencies," Int. J. Med. Robot., vol. 7, no. April, pp. 375-392, 2011.

[53] J. J. Leonard and H. F. Durrant-Whyte, "Mobile robot localization by tracking geometric beacons," IEEE Trans. Robot. Autom., vol. 7, no. 3, pp. 376-382, Jun. 1991.

[54] P. S. Maybeck, "Stochastic models, estimation, and control VOLUME 1," 1979.

[55] R. E. Kalman, “A New Approach to Linear Filtering and Prediction Problems,” 1960.

[56] G. Welch and G. Bishop, "An Introduction to the Kalman Filter."

[57] S. Thrun, "Particle Filters in Robotics," Smithsonian, vol. 1, no. 4, pp. 511-518, 2002.

[58] A. J. Davison, I. D. Reid, N. D. Molton, and O. Stasse, "MonoSLAM: Real-Time Single Camera SLAM."

[59] “Spatial mapping - Mixed Reality | Microsoft Docs.” [Online]. Available: https://docs.microsoft.com/en-us/windows/mixed-reality/spatial-mapping. [Accessed: 17-Apr2019].

[60] "Gaze - Mixed Reality | Microsoft Docs.” [Online]. Available: https://docs.microsoft.com/enus/windows/mixed-reality/gaze. [Accessed: 17-Apr-2019].

[61] “Gestures - Mixed Reality | Microsoft Docs.” [Online]. Available: https://docs.microsoft.com/enus/windows/mixed-reality/gestures. [Accessed: 17-Apr-2019].

[62] R. Vassallo, A. Rankin, E. C. S. Chen, and T. M. Peters, "Hologram stability evaluation for Microsoft HoloLens," 2017, vol. 10136, p. 1013614.

[63] E. Rae, A. Lasso, M. S. Holden, E. Morin, R. Levy, and G. Fichtinger, "Neurosurgical burr hole placement using the Microsoft HoloLens," Med. Imaging 2018 Image-Guided Proced. Robot. Interv. Model., p. 20, 2018.

[64] F. Incekara, M. Smits, C. Dirven, and A. Vincent, "Clinical Feasibility of a Wearable MixedReality Device in Neurosurgery."

[65] J. T. Gibby, S. A. Swenson, S. Cvetko, R. Rao, and R. Javan, "Head-mounted display augmented 
reality to guide pedicle screw placement utilizing computed tomography," Int. J. Comput. Assist. Radiol. Surg., vol. 14, no. 3, pp. 525-535.

[66] Tristan P. C. van Doormaal, "Clinical Accuracy of Holographic Navigation Using Point-Based Registration on Augmented-Reality Glasses," vol. 0, no. 0, pp. 1-6, 2019.

[67] F. Liebmann, S. Roner, M. von Atzigen, D. Scaramuzza, R. Sutter, J. Snedeker, M. Farshad, and P. Fürnstahl, "Pedicle screw navigation using surface digitization on the Microsoft HoloLens," Int. J. Comput. Assist. Radiol. Surg., vol. 14, no. 7, pp. 1157-1165, Jul. 2019.

[68] G. Klein and D. Murray, "Parallel Tracking and Mapping for Small AR Workspaces," in 2007 6th IEEE and ACM International Symposium on Mixed and Augmented Reality, 2007, pp. 1-10.

[69] T. Frantz, B. Jansen, J. Duerinck, and J. Vandemeulebroucke, “Augmenting Microsoft's HoloLens with vuforia tracking for neuronavigation."

[70] J. B. A. Maintz and M. A. Viergever, "A survey of medical image registration," Med. Image Anal., vol. 2, no. 1, pp. 1-36, Mar. 1998.

[71] G. Bourgeois, M. Magnin, A. Morel, S. Sartoretti, T. Huisman, E. Tuncdogan, D. Meier, and D. Jeanmonod, "Accuracy of MRI-guided stereotactic thalamic functional neurosurgery.," Neuroradiology, vol. 41, no. 9, pp. 636-45, Sep. 1999.

[72] R. J. Maciunas, R. L. Galloway, and J. W. Latimer, "The Application Accuracy of Stereotactic Frames," Neurosurgery, vol. 35, no. 4, pp. 682-695, Oct. 1994. 